\title{
Limit cycles bifurcating from a degenerate center
}

\author{
Jaume Llibre ${ }^{1}$ and Chara Pantazi ${ }^{2}$ \\ ${ }^{1}$ Departament de Matemàtiques, Universitat Autònoma de Barcelona, Edifici C, 08193 \\ Bellaterra, Barcelona, Catalonia, Spain \\ jllibre@mat.uab.cat \\ ${ }^{2}$ Departament de Matemàtica Aplicada I, Universitat Politècnica de Catalunya, \\ (EPSEB), Av. Doctor Marañón, 44-50, 08028 Barcelona, Spain \\ chara.pantazi@upc.edu
}

\begin{abstract}
We study the maximum number of limit cycles that can bifurcate from a degenerate center of a cubic homogeneous polynomial differential system. Using the averaging method of second order and perturbing inside the class of all cubic polynomial differential systems we prove that at most three limit cycles can bifurcate from the degenerate center. As far as we know this is the first time that a complete study up to second order in the small parameter of the perturbation is done for studying the limit cycles which bifurcate from the periodic orbits surrounding a degenerate center (a center whose linear part is identically zero) having neither a Hamiltonian first integral nor a rational one. This study needs many computations, which have been verified with the help of the algebraic manipulator Maple.
\end{abstract}

Keywords: polynomial differential systems, centers, limit cycles, averaging theory.

2010 MSC: Primary 37G15, 34C07. Secondary 37C10, 34C05, 34A34

\section{Introduction}

Hilbert in (16) asked for the maximum number of limit cycles which real polynomial differential systems in the plane of a given degree can have. This is actually the well known 16th Hilbert Problem, see for example the surveys $(17 ; 18)$ and references therein. Recall that a limit cycle of a planar polynomial differential system is a periodic orbit of the system isolated in the set of all periodic orbits of the system. 
Poincaré in (22) was the first to introduce the notion of a center for a vector field defined on the real plane. So according to Poincaré a center is a singular point surrounded by a neighborhood filled of periodic orbits with the unique exception of the singular point.

Consider the polynomial differential system

$$
\dot{x}=P(x, y), \quad \dot{y}=Q(x, y),
$$

and as usually we denote by ${ }^{\circ}=d / d t$. Assume that system (1) has a center located at the origin. Then after a linear change of variables and a possible scaling of time system (1) can be written in one of the following forms

$$
\begin{aligned}
& \dot{x}=-y+F_{1}(x, y), \\
& \dot{y}=x+F_{2}(x, y),
\end{aligned} \quad(B) \quad \begin{aligned}
& \dot{x}=y+F_{1}(x, y), \\
& \dot{y}=F_{2}(x, y),
\end{aligned} \quad(C) \quad \begin{aligned}
& \dot{x}=F_{1}(x, y), \\
& \dot{y}=F_{2}(x, y),
\end{aligned}
$$

with $F_{1}$ and $F_{2}$ polynomials without constant and linear terms. When system (1) can be written into the form (A) we say that the center is of linear type. When system (1) can take the form (B) the center is nilpotent, and when system (1) can be transformed into the form (C) the center is degenerate.

Due to the difficulty of this problem mathematicians have consider simpler versions. Thus Arnold (1) considered the weakened 16th Hilbert Problem, which consists in determining an upper bound for the number of limit cycles which can bifurcate from the periodic orbits of a polynomial Hamiltonian center when it is perturbed inside a class of polynomial differential systems, see for instance (9) and the hundred of references quoted therein. It is known that in a neighborhood of a center always there is a first integral, see (21). When this first integral is not polynomial the computations become more difficult. Moreover, if the center is degenerate the computations become even harder.

In the literature we can basically find the following methods for studying the limit cycles that bifurcate from a center:

- The method that uses the Poincaré return map, like the articles $(4 ; 8)$.

- The one that uses the Abelian integrals or Melnikov integrals (note that for systems in the plane the two notions are equivalent), see for example section 5 of Chapter 6 of (2) and section 6 of Chapter 4 of (15). 
- The one that uses the inverse integrating factor, see $(11 ; 12 ; 13 ; 25)$.

- The averaging theory $(6 ; 14 ; 19 ; 23 ; 24)$.

The first two methods provide information about the number of limit cycles whereas the last two methods additionally give the shape of the bifurcated limit cycle up to any order in the perturbation parameter.

Almost all the papers studying how many limit cycles can bifurcate from the periodic orbits of a center, work with centers of linear type. There are very few papers studying this problem for nilpotent or degenerate centers. In fact, for degenerate centers as far as we known the bifurcation of limit cycles from the periodic orbits of a degenerate center only have been studying completely using formulas of first order in the small parameter of the perturbation. Here we will provide a complete study of this problem using formulas of second order, and as it occurs with the formulas of second order applied to linear centers that they provide in general more limit cycles than the formulas of first order, the same occurs for the formulas of second order applied to degenerate centers. Of course, the computations from first order to second order increases almost exponentially.

This paper deals with the weakened 16th Hilbert's problem but perturbing non-Hamiltonian degenerate centers using the technique of the averaging method of second order, see (14), and Section 2 for a summary of the results that we need here.

Since we want to study the perturbation of a degenerate center with averaging of second order, from the homogeneous centers the first ones that are degenerate, are the cubic homogeneous centers, see for instance (7). In this class in (20) the authors studied the perturbation of the following cubic homogeneous center

$$
\dot{x}=-y\left(3 x^{2}+y^{2}\right), \quad \dot{y}=x\left(x^{2}-y^{2}\right),
$$

inside the class of all cubic polynomial differential systems, using averaging theory of first order. Here we study this problem but using averaging theory of second order.

System (2) has a global center at the origin (i.e. all the orbits contained in $\mathbb{R}^{2} \backslash\{(0,0)\}$ are periodic), and it admits the non-rational first integral

$$
H(x, y)=\left(x^{2}+y^{2}\right) \exp \left(-\frac{2 x^{2}}{x^{2}+y^{2}}\right) .
$$


The limit cycles bifurcating from the periodic orbits of the global center (2) have already been studied in the following two results, see (20) and (5), respectively.

Theorem 1. We deal with differential system (2). Then the polynomial differential system

$$
\begin{aligned}
& \dot{x}=-y\left(3 x^{2}+y^{2}\right)+\varepsilon\left(\sum_{0 \leq i+j \leq 3} a_{i j} x^{i} y^{j}\right), \\
& \dot{y}=x\left(x^{2}-y^{2}\right)+\varepsilon\left(\sum_{0 \leq i+j \leq 3} b_{i j} x^{i} y^{j}\right),
\end{aligned}
$$

has at most one limit cycle bifurcating from the periodic orbits of the center of system (2) using averaging theory of first order. Moreover, there are examples with 1 and 0 limit cycles.

Proposition 2. We consider the homogeneous polynomial differential system (2). Let $P_{i}(x, y)$ and $Q_{i}(x, y)$ for $i=1,2$ be polynomials of degree at most 3. Then for convenient polynomials $P_{i}$ and $Q_{i}$, the polynomial differential system

$$
\begin{aligned}
& \dot{x}=-y\left(3 x^{2}+y^{2}\right)+\varepsilon P_{1}(x, y)+\varepsilon^{2} P_{2}(x, y), \\
& \dot{y}=x\left(x^{2}-y^{2}\right)+\varepsilon Q_{1}(x, y)+\varepsilon^{2} Q_{2}(x, y),
\end{aligned}
$$

has at first order averaging one limit cycle, and at second order averaging two limit cycles bifurcating from the periodic solutions of the global center $(2)$.

Our main result is the following one and it do by first time the complete study of the averaging method of second order for a degenerate center having neither a Hamiltonian first integral nor a rational one.

Theorem 3. We consider the cubic homogeneous differential system (2). Then the perturbation of system (2) inside the class of all cubic polynomial systems

$$
\begin{aligned}
& \dot{x}=-y\left(3 x^{2}+y^{2}\right)+\varepsilon\left(\sum_{0 \leq i+j \leq 3} a_{i j} x^{i} y^{j}\right)+\varepsilon^{2}\left(\sum_{0 \leq i+j \leq 3} b_{i j} x^{i} y^{j}\right) \\
& \dot{y}=x\left(x^{2}-y^{2}\right)+\varepsilon\left(\sum_{0 \leq i+j \leq 3} c_{i j} x^{i} y^{j}\right)+\varepsilon^{2}\left(\sum_{0 \leq i+j \leq 3} d_{i j} x^{i} y^{j}\right)
\end{aligned}
$$


has at most three limit cycles bifurcating from the periodic orbits of the center of system (2) using averaging theory of second order. Moreover, there are examples with 3, 2, 1 and 0 limit cycles.

The paper is organized as follows: In section 2 we present a summary of the averaging method of second order following (14). Next in section 3 we provide the proof of our main Theorem 3. In section 4 we provide three examples, of systems (3) with 0, 1, 2 and 3 limit cycles bifurcating from the degenerate center. At the end we present the Appendices A, B and C.

\section{The averaging method of second order}

In this section we present the averaging method of second order following (14). In that paper the averaging theory for differential equations of one variable is done up to any order in the small parameter of the perturbation. We consider the analytic differential equation

$$
\frac{d r}{d \theta}=G_{0}(\theta, r)+\sum_{k \geq 1} \varepsilon^{k} G_{k}(\theta, r),
$$

with $r \in \mathbb{R}, \theta \in \mathbb{S}^{1}$ and $\varepsilon \in\left(-\varepsilon_{0}, \varepsilon_{0}\right)$ with $\varepsilon_{0}$ a small positive real value, and the functions $G_{k}(\theta, r)$ are $2 \pi$-periodic in the variable $\theta$. Note that for $\varepsilon=0$ system (4) is unperturbed. Let $r_{s}\left(\theta, r_{0}\right)$ be the solution of system (4) with $\varepsilon=0$ satisfying $r_{s}\left(0, r_{0}\right)=r_{0}$ and $r_{s}\left(\theta, r_{0}\right)$ is $2 \pi$ periodic for $r_{0} \in \mathcal{I}$ with $\mathcal{I}$ a real open interval. We are interested in the limit cycles of equation (4) which bifurcate from the periodic orbits of the unperturbed system with initial condition $r_{0} \in \mathcal{I}$. So, we define by $r_{\varepsilon}\left(\theta, r_{0}\right)$ the solution of equation (4) satisfying $r_{\varepsilon}\left(0, r_{0}\right)=r_{0}$.

In what follows we denote by $u=u\left(\theta, r_{0}\right)$ the solution of the variational equation

$$
\frac{\partial u}{\partial \theta}=\frac{\partial G_{0}}{\partial r}\left(\theta, r_{s}\left(\theta, r_{0}\right)\right) u
$$

satisfying $u\left(0, r_{0}\right)=1$. 
We define

$$
\begin{aligned}
& u_{1}\left(\theta, r_{0}\right)= \int_{0}^{\theta} \frac{G_{1}\left(\phi, r_{s}\left(\phi, r_{0}\right)\right)}{u\left(\phi, r_{0}\right)} d \phi=\int_{0}^{\theta} \frac{G_{1}\left(w, r_{s}\left(w, r_{0}\right)\right)}{u\left(w, r_{0}\right)} d w, \\
& G_{10}\left(r_{0}\right)= \int_{0}^{2 \pi} \frac{G_{1}\left(\theta, r_{s}\left(\theta, r_{0}\right)\right)}{u\left(\theta, r_{0}\right)} d \theta, \\
& G_{20}\left(r_{0}\right)= \int_{0}^{2 \pi}\left(\frac{G_{2}\left(\theta, r_{s}\left(\theta, r_{0}\right)\right)}{u\left(\theta, r_{0}\right)}+\frac{\partial G_{1}}{\partial r}\left(\theta, r_{s}\left(\theta, r_{0}\right)\right) u_{1}\left(\theta, r_{0}\right)\right. \\
&\left.\quad+\frac{1}{2} \frac{\partial^{2} G_{0}}{\partial r^{2}}\left(\theta, r_{s}\left(\theta, r_{0}\right)\right) u_{1}\left(\theta, r_{0}\right)^{2}\right) d \theta .
\end{aligned}
$$

In statement (b) of Corollary 5 of (14) it is proved the following result.

Theorem 4. Assume that the solution $r_{s}\left(\theta, r_{0}\right)$ of the unperturbed equation (4) such that $r_{s}\left(0, r_{0}\right)=r_{0}$ is $2 \pi$-periodic for $r_{0} \in \mathcal{I}$ with $\mathcal{I}$ a real open interval. If $G_{10}\left(r_{0}\right)$ is identically zero in $\mathcal{I}$ and $G_{20}\left(r_{0}\right)$ is not identically zero

in $\mathcal{I}$, then for each simple zero $r^{*} \in \mathcal{I}$ of $G_{20}\left(r_{0}\right)=0$ there exists a periodic solution $r_{\varepsilon}\left(\theta, r_{0}\right)$ of (4) such that $r_{\varepsilon}\left(0, r_{0}\right) \rightarrow r^{*}$ when $\varepsilon \rightarrow 0$.

\section{Proof of Theorem 3}

System (2) in polar coordinates becomes

$$
\dot{r}=-2 r^{3} \cos \theta \sin \theta, \quad \dot{\theta}=r^{2},
$$

or equivalently,

$$
\frac{d r}{d \theta}=-2 r \cos \theta \sin \theta
$$

and it has the solution $r_{s}\left(\theta, r_{0}\right)=r_{0} \exp \left(-\sin ^{2} \theta\right)$ satisfying that $r_{s}\left(0, r_{0}\right)=$ $r_{0}$.

Now we perturb system (2) inside the class of all cubic polynomial differential systems as in (3). System (3) in polar coordinates give rise to the differential equation

$$
\frac{d r}{d \theta}=G_{0}(\theta, r)+\varepsilon G_{1}(\theta, r)+\varepsilon^{2} G_{2}(\theta, r)+O\left(\varepsilon^{3}\right)
$$


with

$$
\begin{aligned}
G_{0}(\theta, r)= & -2 r \cos \theta \sin \theta \\
G_{1}(\theta, r)= & g_{1,1}(\theta) \frac{1}{r^{2}}+g_{1,2}(\theta) \frac{1}{r}+g_{1,3}(\theta)+g_{1,4}(\theta) r, \\
G_{2}(\theta, r)= & g_{2,1}(\theta) \frac{1}{r^{5}}+g_{2,2}(\theta) \frac{1}{r^{4}}+g_{2,3}(\theta) \frac{1}{r^{3}}+g_{2,4}(\theta) \frac{1}{r^{2}} \\
& +g_{2,5}(\theta) \frac{1}{r}+g_{2,6}(\theta)+g_{2,7}(\theta) r,
\end{aligned}
$$

where the expressions of the coefficients $g_{1, i}(\theta)$ for $i=1,2,3,4$ and $g_{2, j}(\theta)$ for $j=1,2, \cdots, 7$ are given in the Appendix A.

Additionally, we consider the variational equation

$$
\frac{\partial u}{\partial \theta}=\frac{\partial G_{0}}{\partial r}\left(\theta, r_{s}\left(\theta, r_{0}\right)\right)
$$

and its solution $u\left(\theta, r_{0}\right)$ satisfying $u\left(0, r_{0}\right)=1$, namely $u_{s}(\theta)=\exp \left(-\sin ^{2} \theta\right)$.

We define

$$
\begin{aligned}
& I_{1}=\int_{0}^{2 \pi} \exp \left(2 \sin ^{2} \theta\right) \cos ^{4} \theta d \theta=3.572403292 \ldots, \\
& I_{2}=\int_{0}^{2 \pi} \exp \left(2 \sin ^{2} \theta\right) \cos ^{2} \theta d \theta=5.985557563 \ldots, \\
& I_{3}=\int_{0}^{2 \pi} \exp \left(2 \sin ^{2} \theta\right) d \theta=21.62373221 \ldots
\end{aligned}
$$

Lemma 5. Consider $I_{1}, I_{2}, I_{3}$ defined in (7). Then for $a_{10}=-\left(I_{3}-2 I_{1}+\right.$ $\left.I_{2}\right) /\left(2 I_{1}-I_{2}\right) c_{01}$ and $a_{30}=-2 c_{03}-c_{21}$ we have that the function $G_{10}\left(r_{0}\right)$ defined in (5) is identically zero.

Proof. We have

$$
\frac{G_{1}\left(\theta, r_{s}\left(\theta, r_{0}\right)\right)}{u\left(\theta, r_{0}\right)}=A(\theta) \frac{1}{r_{0}^{2}}+B(\theta) \frac{1}{r_{0}}+C(\theta)+D(\theta) r_{0}
$$


with

$$
\begin{aligned}
& A(\theta)=\left[\sin \theta\left(2 \cos ^{2} \theta+1\right) c_{00}+\cos \theta\left(-1+2 \cos ^{2} \theta\right) a_{00}\right] \mathrm{e}^{3 \sin ^{2} \theta} \\
& B(\theta)=\left[\cos \theta \sin \theta\left(-1+2 \cos ^{2} \theta\right) a_{01}+\cos ^{2} \theta\left(-1+2 \cos ^{2} \theta\right) a_{10}\right. \\
& \left.-\left(-\cos ^{2} \theta+2 \cos ^{4} \theta-1\right) c_{01}+\cos \theta \sin \theta\left(2 \cos ^{2} \theta+1\right) c_{10}\right] \mathrm{e}^{2} \sin ^{2} \theta \\
& C(\theta)=\left[-\cos \theta\left(-3 \cos ^{2} \theta+1+2 \cos ^{4} \theta\right) a_{02}+\cos ^{3} \theta\left(-1+2 \cos ^{2} \theta\right) a_{20}\right. \\
& +\cos ^{2} \theta \sin \theta\left(-1+2 \cos ^{2} \theta\right) a_{11}+\sin ^{3} \theta\left(2 \cos ^{2} \theta+1\right) c_{02} \\
& \left.+\cos ^{2} \theta \sin \theta\left(2 \cos ^{2} \theta+1\right) c_{20}-\cos \theta\left(-\cos ^{2} \theta+2 \cos ^{4} \theta-1\right) c_{11}\right] \mathrm{e}^{\sin ^{2} \theta} \\
& D(\theta)=\cos \theta \sin ^{3} \theta\left(-1+2 \cos ^{2} \theta\right) a_{03}+\cos ^{4} \theta\left(-1+2 \cos ^{2} \theta\right) a_{30} \\
& +\cos ^{3} \theta \sin \theta\left(-1+2 \cos ^{2} \theta\right) a_{21}-\cos ^{2} \theta\left(-3 \cos ^{2} \theta+1+2 \cos ^{4} \theta\right) a_{12} \\
& +\left(1-3 \cos 4 \theta+2 \cos ^{6} \theta\right) c_{03}+\cos ^{3} \theta \sin \theta\left(2 \cos ^{2} \theta+1\right) c_{30} \\
& -\cos ^{2} \theta\left(-\cos ^{2} \theta+2 \cos ^{4} \theta-1\right) c_{21}+\cos \theta \sin ^{3} \theta\left(2 \cos ^{2} \theta+1\right) c_{12} .
\end{aligned}
$$

Now

$G_{10}\left(r_{0}\right)=\int_{0}^{2 \pi} \frac{G_{1}\left(\theta, r_{s}\left(\theta, r_{0}\right)\right)}{u\left(\theta, r_{0}\right)} d \theta=\int_{0}^{2 \pi}\left(A(\theta) \frac{1}{r_{0}^{2}}+B(\theta) \frac{1}{r_{0}}+C(\theta)+D(\theta) r_{0}\right) d \theta$

and considering the change of coordinates $\theta=\phi+\pi$ in the interval $[0,2 \pi]$ and the symmetries

$$
\sin (\theta+\pi)=-\sin \theta, \quad \cos (\theta+\pi)=-\cos \theta
$$

we have that

$$
\int_{0}^{2 \pi} A(\theta) d \theta=\int_{-\pi}^{\pi} A(\phi) d \phi=0, \quad \int_{0}^{2 \pi} C(\theta) d \theta=\int_{-\pi}^{\pi} C(\phi) d \phi=0 .
$$

So we have

$$
\begin{aligned}
G_{10}\left(r_{0}\right) & =\int_{0}^{2 \pi} \frac{B(\theta)}{r_{0}} d \theta+\int_{0}^{2 \pi} D(\theta) r_{0} d \theta \\
& =\left[\left(2 a_{10}-2 c_{01}\right) I_{1}+\left(c_{01}-a_{10}\right) I_{2}+c_{01} I_{3}\right] \frac{1}{r_{0}}+\frac{\pi}{2}\left(2 c_{03}+a_{30}+c_{21}\right) r_{0}
\end{aligned}
$$


and therefore $G_{10} \equiv 0$ if

$$
a_{10}=-\frac{I_{3}-2 I_{1}+I_{2}}{2 I_{1}-I_{2}} c_{01}=-17.65322447 . . c_{01}, \quad a_{30}=-2 c_{03}-c_{21} .
$$

This completes the proof of the lemma.

Now we have

$\frac{G_{2}\left(\theta, r_{s}\left(\theta, r_{0}\right)\right)}{u\left(\theta, r_{0}\right)}=A_{5}(\theta) \frac{1}{r_{0}^{5}}+A_{4}(\theta) \frac{1}{r_{0}^{4}}+A_{3}(\theta) \frac{1}{r_{0}^{3}}+A_{2}(\theta) \frac{1}{r_{0}^{2}}+A_{1}(\theta) \frac{1}{r_{0}}+A_{0}(\theta)+\tilde{A}_{1}(\theta) r_{0}$,

with $A_{5}(\theta), A_{4}(\theta), A_{3}(\theta), A_{2}(\theta), A_{1}(\theta), A_{0}(\theta), \tilde{A}_{1}(\theta)$ are given in the Appendix B.

We note that

$$
\int_{0}^{2 \pi} A_{4}(\theta) d \theta=0, \quad \int_{0}^{2 \pi} A_{2}(\theta) d \theta=0, \quad \int_{0}^{2 \pi} A_{0}(\theta) d \theta=0
$$

because of the symmetries (8). So we have

$$
\int_{0}^{2 \pi} \frac{G_{2}\left(\theta, r_{s}\left(\theta, r_{0}\right)\right)}{u\left(\theta, r_{0}\right)} d \theta=\frac{1}{r_{0}^{5}} \int_{0}^{2 \pi} A_{5}(\theta) d \theta+\frac{1}{r_{0}^{3}} \int_{0}^{2 \pi} A_{3}(\theta) d \theta+\frac{1}{r_{0}} \int_{0}^{2 \pi} A_{1}(\theta) d \theta+\left(\int_{0}^{2 \pi} \tilde{A}_{1}(\theta) d \theta\right) r_{0},
$$

and we recall that the expressions of $A_{5}(\theta), A_{3}(\theta), A_{1}(\theta), \tilde{A}_{1}(\theta)$ are given in the Appendix B. We have

$$
\begin{aligned}
\int_{0}^{2 \pi} A_{5}(\theta) d \theta & =\left(-4 \int_{0}^{2 \pi} \mathrm{e}^{6 \sin ^{2} \theta} \cos ^{4} \theta d \theta+2 \int_{0}^{2 \pi} \mathrm{e}^{6 \sin ^{2} \theta} \cos ^{2} \theta d \theta+\int_{0}^{2 \pi} \mathrm{e}^{6 \sin ^{2} \theta} d \theta\right) a_{00} c_{00} \\
& =665.2264930 . . a_{00} c_{00}, \\
\int_{0}^{2 \pi} A_{3}(\theta) d \theta & =239.0000390 . . a_{01} c_{01}+97.83745135 . . a_{00} c_{02}+97.83745135 . . a_{02} c_{00} \\
& -257.2692783 . . c_{01} c_{10}+12.93483815 . . a_{00} c_{20}-7.99641945 . . a_{00} a_{11} \\
& +12.93483815 . . a_{20} c_{00}-28.92767705 . . c_{00} c_{11},
\end{aligned}
$$




$$
\begin{aligned}
\int_{0}^{2 \pi} A_{1}(\theta) d \theta & =1.159249021 . . b_{10}+20.46448319 . . d_{01}+2.318498043 . . a_{11} c_{11} \\
& -4.73165232 . . c_{02} c_{11}+2.318498043 . . a_{20} c_{0,2}+2.318498045 . . a_{02} c_{20} \\
& -3.761715750 . . c_{11} c_{20}+14.47892563 . . a_{02} c_{02}-1.253905250 . . a_{02} a_{11} \\
& +0.189312456 . . a_{20} c_{20}+0.094656226 . . a_{11} a_{20}+0.647510463 . . a_{21} c_{01} \\
& -5.110277230 . . c_{03} c_{10}+2.318498043 . . a_{12} c_{10}+2.22384182 . . a_{01} c_{21} \\
& +36.6143963 . . a_{03} c_{01}-3.95102821 . . c_{10} c_{21}-1.25390525 . . a_{01} a_{12} \\
& -45.6606188 . . c_{01} c_{12}-7.1036910 . . c_{01} c_{30}+14.28961317 . . a_{01} c_{03}, \\
\int_{0}^{2 \pi} \tilde{A}_{1}(\theta) d \theta & =-0.3926990817 . . . c_{30} c_{21}+0.1963495408 . . c_{30} a_{12}+2.159844949 . . a_{03} c_{03} \\
& -0.1963495408 . . a_{03} a_{12}-0.7853981634 . . c_{12} c_{21}+0.3926990817 . . a_{03} c_{21} \\
& -1.178097245 . . c_{03} c_{12}+0.3926990817 . . c_{12} a_{12}+0.9817477042 . . c_{03} c_{30} \\
& +1.570796327 . . d_{21}+3.141592654 . . d_{03}+1.570796327 . . b_{30} .
\end{aligned}
$$

Remark 6. (a) Looking at the expressions of $A_{3}, A_{1}, \tilde{A}_{1}$ in the Appendix $B$ we can have the exact definition for the numerical coefficients which appear in the previous integrals. Thus for instance

$$
239.0000390 \cdots=-\int_{0}^{2 \pi} \frac{\mathrm{e}^{4 \sin ^{2} \theta}\left(\cos ^{2} \theta-1\right)\left(2 I_{1}-4 I_{3} \cos ^{4} \theta+2 I_{3} \cos ^{2} \theta-I_{2}\right)}{2 I_{1}-I_{2}} d \theta,
$$

and $I_{1}, I_{2}, I_{3}$ satisfying relations $(7)$.

(b) All the computations of this paper have been verified with the algebraic manipulator Maple.

We additionally have

$$
\frac{\partial G_{1}}{\partial r}\left(\theta, r_{s}\left(\theta, r_{0}\right)\right)=B_{0}(\theta)+\frac{B_{1}(\theta)}{r_{0}^{2}}+\frac{B_{2}(\theta)}{r_{0}^{3}}
$$

with

$$
\begin{aligned}
B_{0}(\theta)= & \cos ^{2} \theta\left(1+2 \cos ^{2} \theta-4 \cos ^{4} \theta\right) c_{21} \\
& +\left(-2 \cos ^{6} \theta+1-\cos ^{4} \theta\right) c_{03} \\
& +\cos \theta\left(\sin \theta \cos ^{2} \theta-2 \cos ^{4} \theta \sin \theta+\sin \theta\right) c_{12} \\
& +\cos ^{3} \theta\left(\sin \theta+2 \cos ^{2} \theta \sin \theta\right) c_{30} \\
& +\sin \theta \cos \theta\left(3 \cos ^{2} \theta-1-2 \cos ^{4} \theta\right) a_{03} \\
& +\cos ^{2} \theta\left(-1+3 \cos ^{2} \theta-2 \cos ^{4} \theta\right) a_{12} \\
& +\sin \theta \cos ^{3} \theta\left(-1+2 \cos ^{2} \theta\right) a_{21}
\end{aligned}
$$




$$
\begin{aligned}
B_{1}(\theta)= & \left(-1-18.65322447 \ldots \cos ^{2} \theta+37.30644894 \ldots \cos ^{4} \theta\right) \mathrm{e}^{2 \sin ^{2} \theta} c_{01} \\
& -\sin \theta \cos \theta\left(2 \cos ^{2} \theta+1\right) \mathrm{e}^{2 \sin ^{2} \theta} c_{10} \\
& +\sin \theta \cos \theta\left(-2 \cos ^{2} \theta+1\right) \mathrm{e}^{2 \sin ^{2} \theta} a_{01}, \\
B_{2}(\theta)= & -2 \sin \theta\left(2 \cos ^{2} \theta+1\right) c_{00}+2\left(-2+\mathrm{e}^{3 \sin ^{2} \theta} \cos \theta\right) a_{00} .
\end{aligned}
$$

Now we have

$$
\frac{G_{1}\left(w, r_{s}\left(w, r_{0}\right)\right)}{u\left(w, r_{0}\right)}=\frac{C_{2}(w)}{r_{0}^{2}}+\frac{C_{1}(w)}{r_{0}}+C_{0}(w)+\tilde{C}_{1}(w) r_{0}
$$

with

$$
\begin{aligned}
C_{2}(w)= & \mathrm{e}^{3 \sin ^{2} w}\left[\cos w\left(2 \cos ^{2} w-1\right) a_{00}+\sin w\left(1+2 \cos ^{2} w\right) c_{00}\right] \\
C_{1}(w)= & {\left[\cos w \sin w\left(-1+2 \cos ^{2} w\right) a_{01}\right.} \\
& +\left(1+\frac{I_{3}}{2 I_{1}-I_{2}}\left(\cos ^{2} w-2 \cos ^{4} w\right)\right) c_{01} \\
& \left.+\cos w \sin w\left(1+2 \cos ^{2} w\right) c_{10}\right] \mathrm{e}^{2} \sin ^{2} w \\
C_{0}(w)= & \cos ^{3} w\left(2 \cos ^{2} w-1\right) a_{20}-\cos w\left(-3 \cos ^{2} w+2 \cos ^{4} w+1\right) a_{02} \\
& +\sin w \cos ^{2} w\left(2 \cos ^{2} w-1\right) a_{11}+\sin w \cos ^{2} w\left(1+2 \cos ^{2} w\right) c_{20} \\
& \left.+\cos _{02} \sin ^{3} w\left(1+2 \cos ^{2} w\right)-\cos w\left(2 \cos ^{4} w-1-\cos ^{2} w\right) c_{11}\right] \mathrm{e}^{\sin ^{2} w}, \\
\tilde{C}_{1}(w)= & -\left(\cos ^{4} w+2 \cos ^{6} w-1\right) c_{03}+\sin ^{3} w \cos ^{2} w\left(1+2 \cos ^{2} w\right) c_{12} \\
& \left.-\cos ^{2} w\left(-1-2 \cos ^{2} w+4 \cos ^{4} w\right) c_{21}+\cos ^{3} w \sin ^{2} w+2 \cos ^{2} w\right) c_{30} \\
& +\sin ^{3} w \cos w\left(2 \cos ^{2} w-1\right) a_{03}+\sin ^{2} w \cos ^{2} w\left(2 \cos ^{2} w-1\right) a_{12} \\
& +\cos ^{3} w \sin w\left(2 \cos ^{2} w-1\right) a_{21} \cdot
\end{aligned}
$$

Additionally, from (5) we obtain

$$
u_{1}\left(\theta, r_{0}\right)=\frac{1}{r_{0}^{2}} \int_{0}^{\theta} C_{2}(w) d w+\frac{1}{r_{0}} \int_{0}^{\theta} C_{1}(w) d w+\int_{0}^{\theta} C_{0}(w) d w+r_{0} \int_{0}^{\theta} \tilde{C}_{1}(w) d w
$$

and so

$$
\frac{\partial G_{1}}{\partial r}\left(\theta, r_{s}\left(\theta, r_{0}\right)\right) u_{1}\left(\theta, r_{0}\right)=s_{5}(\theta) \frac{1}{r_{0}^{5}}+s_{4}(\theta) \frac{1}{r_{0}^{4}}+s_{3}(\theta) \frac{1}{r_{0}^{3}}+s_{2}(\theta) \frac{1}{r_{0}^{2}}+s_{1}(\theta) \frac{1}{r_{0}}+s_{0}(\theta)+\tilde{s}_{1}(\theta) r_{0},
$$


and the explicit expressions of $s_{i}(\theta)$ for $i=0,1, \cdots, 5$ and $\tilde{s}_{1}(\theta)$ are given in the Appendix C.

Since $\frac{\partial^{2} G_{0}}{\partial r^{2}}=0$ from (5) we have that

$$
G_{20}\left(r_{0}\right)=\int_{0}^{2 \pi}\left(\frac{G_{2}\left(\theta, r_{s}\left(\theta, r_{0}\right)\right)}{u\left(\theta, r_{0}\right)}+\frac{\partial G_{1}}{\partial r}\left(\theta, r_{s}\left(\theta, r_{0}\right)\right) u_{1}\left(\theta, r_{0}\right)\right) d \theta \text {, }
$$

and we obtain

$$
r_{0}^{5} G_{20}\left(r_{0}\right)=v_{6} r_{0}^{6}+v_{4} r_{0}^{4}+v_{2} r_{0}^{2}+v_{0}
$$

with

$$
\begin{aligned}
v_{6}= & -0.3926990800 \cdots c_{21} c_{30}+0.1963495397 \cdots a_{12} c_{30}+2.159844949 \cdots a_{03} c_{03} \\
& -0.1963495365 \cdots a_{03} a_{12}-0.7853981634 \cdots c_{12} c_{21}+0.3926990817 \cdots a_{03} c_{21} \\
& -1.178097245 \cdots c_{03} c_{12}+0.3926990817 \cdots a_{12} c_{12}+0.9817477042 \cdots c_{03} c_{30} \\
& +1.570796327 \cdots d_{21}+3.141592654 \cdots d_{03}+1.570796327 \cdots b_{30}, \\
v_{4}= & -3.155691751 \cdots a_{21} c_{01}+6.612510180 \cdots c_{03} c_{10}+1.786201647 \cdots a_{12} c_{10} \\
& +2.413154277 \cdots a_{01} c_{21}+38.88726613 \cdots a_{03} c_{01}-1.253905255 \cdots c_{10} c_{21} \\
& -1.206577137 \cdots a_{01} a_{12}-68.30745733 \cdots c_{01} c_{12}-38.88726635 \cdots c_{01} c_{30} \\
& +13.27234849 \cdots a_{01} c_{03}+2.318498043 \cdots a_{11} c_{11}-4.73165232 \cdots c_{02} c_{11} \\
& +2.318498043 \cdots a_{20} c_{02}+2.318498045 \cdots a_{02} c_{20}-3.761715750 \cdots c_{11} c_{20} \\
& +14.47892563 \cdots a_{02} c_{02}-1.253905250 \cdots a_{02} a_{11}+0.189312456 \cdots a_{20} c_{20} \\
& +0.094656226 \cdots a_{11} a_{20}+1.159249021 \cdots b_{10}+20.46448319 \cdots d_{01}, \\
v_{2}= & 95.95703341 \cdots a_{00} c_{02}+105.7377762 \cdots a_{02} c_{00}+239.0000390 \cdots a_{01} c_{01} \\
& -7.649140220 \cdots a_{00} a_{11}+16.68739736 \cdots a_{00} c_{20}-110.9164314 \cdots c_{00} c_{11} \\
& -257.2692783 \cdots c_{01} c_{10}-0.000001 \cdots c_{01}{ }^{2}-31.79348852 \cdots a_{20} c_{00}, \\
v_{0}= & 665.2264933 \cdots a_{00} c_{00} .
\end{aligned}
$$

We have that the coefficients $v_{6}, v_{4}, v_{2}, v_{0}$ are independent because $d_{03}$ only appears in $v_{6}, b_{10}$ only appears in $v_{4}, a_{00} c_{02}$ only appears in $v_{2}$, and $a_{00} c_{00}$ only appears in $v_{0}$.

Now we are going to use Descartes Theorem:

Theorem 7 (Descartes Theorem). Consider the real polynomial $p(x)=$ $a_{i_{1}} x^{i_{1}}+a_{i_{2}} x^{i_{2}} \cdots+a_{i_{r}} x^{i_{r}}$ with $0 \leq i_{1}<i_{2}<\cdots<i_{r}$ and $a_{i_{j}} \neq 0$ real 
constants for $j \in\{1,2, \cdots, r\}$. When $a_{i_{j}} a_{i_{j+1}}<0$, we say that $a_{i_{j}}$ and $a_{i_{j+1}}$ have a variation of sign. If the number of variations of signs is $m$, then $p(x)$ has at most $m$ positive real roots. Moreover, it is always possible to choose the coefficients of $p(x)$ in such a way that $p(x)$ has exactly $r-1$ positive real roots.

For a proof of Descartes Theorem see pages 82-83 of (3).

So from Descartes Theorem we can choose $v_{6}, v_{4}, v_{2}, v_{0}$ in order that the $G_{20}$ has 3,2,1 or 0 real positive roots. This completes the proof of the first part of Theorem 3 .

Remark 8. Again the exact definition for the numerical coefficients which appear in $v_{6}, v_{4}, v_{2}$ and $v_{0}$ are given in Appendices $B$ and $C$. For instance

$$
v_{0}=\int_{0}^{2 \pi} A_{5} d \theta+\int_{0}^{2 \pi} s_{5,3} d \theta=\int_{0}^{2 \pi} A_{5} d \theta=665.2264933 \ldots
$$

For completing the proof of Theorem 3 we shall provide examples of system (3) with 3,2, 1 and 0 limit cycles. In fact, strictly speaking it is not necessary to provide examples with $3,2,1$ and 0 limit cycles but we want to provide such examples.

\section{Examples}

\section{Example with 3 limit cycles}

In Figure 1 we see that for $\varepsilon=0.001$ the system

$$
\begin{aligned}
\dot{x}= & -y\left(3 x^{2}+y^{2}\right)+\varepsilon+\varepsilon^{2}\left(3570.576292 x-752.8823806 x^{3}\right) \\
& =y\left(3 x^{2}+y^{2}\right)+0.001+0.003570576292 x-0.0007528823806 x^{3}, \\
\dot{y}= & x\left(x^{2}-y^{2}\right)+\varepsilon\left(1-37.74385845 y^{2}\right) \\
& =x\left(x^{2}-y^{2}\right)+0.001-0.03774385845 y^{2},
\end{aligned}
$$

has three limit cycles, since for system (11) we have

$G_{20}\left(r_{0}\right)=-1182.624878 r_{0}+4139.187071 \frac{1}{r_{0}}-3621.788686 \frac{1}{r_{0}^{3}}+665.2264933 \frac{1}{r_{0}^{5}}$,

and from $G_{20}\left(r_{0}\right)=0$ we obtain the three positive roots near to $r_{0}=$ $0.5,1,1.5$. 


$$
\varepsilon=0 \quad \varepsilon=0.001
$$

Figure 1: For $\varepsilon=0$ we have the degenerate center of system (2), and for $\varepsilon=0.001$ the perturbed system (11) has three limit cycles.

We have used the program P4 described in Chapters 9 and 10 of (10) for doing the phase portraits in the Poincaré disc which appear in this paper.

\section{Example with 2 limit cycles}

For $\varepsilon=0.001$ the system

$$
\begin{aligned}
\dot{x}= & -y\left(3 x^{2}+y^{2}\right)+\varepsilon\left(1+y+x^{2} y\right)+\varepsilon^{2}\left(-856.6373973 x+y^{3}\right) \\
& =-y\left(3 x^{2}+y^{2}\right)+0.001+0.001 y+0.001 x^{2} y-0.0008566373973 x+0.000001 y^{3}, \\
\dot{y}= & x\left(x^{2}-y^{2}\right)+\varepsilon\left(1+y^{2}\right)+\varepsilon^{2}\left(x+73.80732101 y^{3}\right) \\
& =x\left(x^{2}-y^{2}\right)+0.001+0.001 y^{2}+0.000001 x+0.00007380732101 y^{3},
\end{aligned}
$$

gives

$G_{20}\left(r_{0}\right)=231.8725375 r_{0}-993.0560642 \frac{1}{r_{0}}+95.95703341 \frac{1}{r_{0}^{3}}+665.2264933 \frac{1}{r_{0}^{5}}$,

and $G_{20}\left(r_{0}\right)=0$ has the two positive zeros $r_{0}=1$ and $r_{0}=2$. In Figure 2 we see the two limit cycles bifurcated from the degenerate center of the unperturbed system (12).

$$
\varepsilon=0.001
$$

Figure 2: Two limit cycles bifurcate from the degenerate center of the unperturbed system (12).

\section{Example with 1 limit cycle}

For $\varepsilon=0.001$ the system

$$
\begin{aligned}
\dot{x} & =-y\left(3 x^{2}+y^{2}\right)+\varepsilon(1-176.5322447 x)+\varepsilon^{2}\left(x+x^{3}\right) \\
& =-y\left(3 x^{2}+y^{2}\right)+0.001-0.1765312447 x+0.000001 x^{3}, \\
\dot{y} & =x\left(x^{2}-y^{2}\right)+\varepsilon(10+10 y+5 x y)+\varepsilon^{2}\left(y-y^{3}\right) \\
& =x\left(x^{2}-y^{2}\right)+0.010+0.010001 y+0.005 x y-0.000001 y^{3},
\end{aligned}
$$


has

$G_{20}\left(r_{0}\right)=-1.570796327 r_{0}+21.62373221 \frac{1}{r_{0}}-5545.821670 \frac{1}{r_{0}^{3}}+6652.264933 \frac{1}{r_{0}^{5}}$.

From relation $G_{20}\left(r_{0}\right)=0$ we obtain $-1.097575824,1.097575824,-5.725902515-$

$5.148324797 i, 5.725902515+5.148324797 i,-5.725902515+5.148324797 i, 5.725902515-$ $5.148324797 i$. So only one limit cycle can bifurcate from a periodic orbit of the center of the unperturbed system (13), as we can see in Figure 3.

$$
\varepsilon=0.001
$$

Figure 3: The limit cycle bifurcated from the degenerate center of the unperturbed system (13).

\section{Example with zero limit cycles}

Now for $\varepsilon=0.001$ we consider system

$$
\begin{aligned}
\dot{x} & =-y\left(3 x^{2}+y^{2}\right)+\varepsilon+\varepsilon^{2} x \\
& =-y\left(3 x^{2}+y^{2}\right)+0.001+0.000001 x, \\
\dot{y} & =x\left(x^{2}-y^{2}\right)+\varepsilon\left(1+y^{2}\right)+\varepsilon^{2} y^{3} \\
& =x\left(x^{2}-y^{2}\right)+0.001+0.001 y^{2}+0.000001 y^{3},
\end{aligned}
$$

with

$G_{20}\left(r_{0}\right)=3.141592654 r_{0}+1.159249021 \frac{1}{r_{0}}+95.95703341 \frac{1}{r_{0}^{3}}+665.2264933 \frac{1}{r_{0}^{5}}$.

We have that $G_{20}(r 0)=0$ has solutions $-2.116012294-1.570359831 i, 2.116012294+$ $1.570359831 i,-2.095699520 i, 2.095699520 i,-2.116012294+1.570359831 i, 2.116012294-$ $1.570359831 i$. So no limit cycles can bifurcate from the degenerate center, see also Figure 4. 


$$
\varepsilon=0.001
$$

Figure 4: No limit cycle bifurcates from the degenerate center of the unperturbed system (14).

\section{Appendix A}

$$
\begin{aligned}
& g_{1,1}(\theta)=\cos \theta\left(2 \cos ^{5} \theta-1\right) a_{00}+\sin \theta\left(1+2 \cos ^{2} \theta\right)^{2} c_{00}, \\
& g_{1,2}(\theta)=\sin ^{2} \theta\left(1+2 \cos ^{2} \theta\right) c_{01}+\sin \theta \cos \theta\left(1+2 \cos ^{2} \theta\right) c_{10} \\
& +\sin \theta \cos \theta\left(2 \cos ^{2} \theta-1\right) a_{01}+\cos ^{2} \theta\left(2 \cos ^{2} \theta-1\right) a_{10}, \\
& g_{1,3}(\theta)=\sin ^{3} \theta\left(1+2 \cos ^{2} \theta\right) c_{02}+\left(-2 \cos ^{5} \theta+\cos ^{3} \theta+\cos \theta\right) c_{11} \\
& +\cos ^{2} \theta \sin \theta\left(1+2 \cos ^{2} \theta\right) c_{20}+\left(-2 \cos ^{5} \theta+3 \cos ^{3} \theta-\cos \theta\right) a_{02}+ \\
& \cos ^{2} \theta \sin \theta\left(2 \cos ^{2} \theta-1\right) a_{11}+\cos ^{3} \theta\left(2 \cos ^{2} \theta-1\right) a_{20}, \\
& g_{1,4}(\theta)=\sin ^{4} \theta\left(1+2 \cos ^{2} \theta\right) c_{03}+\sin ^{3} \theta \cos \theta\left(1+2 \cos ^{2} \theta\right) c_{12} \\
& +\sin ^{2} \theta \cos ^{2} \theta\left(1+2 \cos ^{2} \theta\right) c_{21}+\sin \theta \cos ^{3} \theta\left(1+2 \cos ^{2} \theta\right) c_{30} \\
& +\sin ^{3} \theta \cos \theta\left(2 \cos ^{2} \theta-1\right) a_{03}+\sin ^{2} \theta \cos ^{2} \theta\left(2 \cos ^{2} \theta-1\right) a_{12} \\
& +\sin \theta \cos ^{3} \theta\left(2 \cos ^{2} \theta-1\right) a_{21}+\cos ^{4} \theta\left(2 \cos ^{2} \theta-1\right) a_{30}, \\
& g_{2,1}(\theta)=\left(2 \cos ^{2} \theta+1-4 \cos ^{4} \theta\right) a_{00} c_{00}-c_{00}^{2} \sin \theta \cos \theta\left(2 \cos ^{2} \theta+1\right) c_{00}^{2} \\
& +\sin \theta \cos \theta\left(2 \cos ^{2} \theta-1\right) a_{00}{ }^{2} \text {, } \\
& g_{2,2}(\theta)=-\cos \theta\left(-2 \cos ^{2} \theta-1+4 \cos ^{4} \theta\right) c_{10} a_{00}-2 \cos ^{2} \theta \sin \theta\left(2 \cos ^{2} \theta+1\right) c_{10} c_{00} \\
& +2 \cos ^{2} \theta \sin \theta\left(2 \cos ^{2} \theta-1\right) a_{10} a_{00}-\sin \theta\left(-2 \cos ^{2} \theta-1+4 \cos ^{4} \theta\right) c_{01} a_{00} \\
& -\sin \theta\left(-2 \cos ^{2} \theta-1+4 \cos ^{4} \theta\right) c_{00} a_{01}-\cos \theta\left(-2 \cos ^{2} \theta-1+4 \cos ^{4} \theta\right) c_{00} a_{10} \\
& +\left(4 \cos ^{5} \theta-2 \cos \theta-2 \cos ^{3} \theta\right) c_{01} c_{00}+\left(6 \cos ^{3} \theta-4 \cos ^{5} \theta-2 \cos \theta\right) a_{01} a_{00} \text {, } \\
& g_{2,3}(\theta)=\left(\cos ^{2} \theta+4 \cos ^{6} \theta-6 \cos ^{4} \theta+1\right) a_{01} c_{01}+\left(\cos ^{2} \theta+4 \cos ^{6} \theta-6 \cos ^{4} \theta+1\right) a_{00} c_{02} \\
& +\left(\cos ^{2} \theta+4 \cos ^{6} \theta-6 \cos ^{4} \theta+1\right) a_{02} c_{00}-c_{11} a_{00} \sin \theta \cos \theta\left(-2 \cos ^{2} \theta-1+4 \cos ^{4} \theta\right) \\
& -c_{00} a_{11} \sin \theta \cos \theta\left(-2 \cos ^{2} \theta-1+4 \cos ^{4} \theta\right)-2 c_{20} c_{00} \sin \theta \cos ^{3} \theta\left(2 \cos ^{2} \theta+1\right) \\
& +2 a_{20} a_{00} \sin \theta \cos ^{3} \theta\left(2 \cos ^{2} \theta-1\right)+2 a_{02} a_{00} \sin ^{3} \theta \cos \theta\left(2 \cos ^{2} \theta-1\right) \\
& -2 c_{02} c_{00} \sin ^{3} \theta \cos \theta\left(2 \cos ^{2} \theta+1\right)-c_{10} a_{01} \sin \theta \cos \theta\left(-2 \cos ^{2} \theta-1+4 \cos ^{4} \theta\right) \\
& -c_{01} a_{10} \sin \theta \cos \theta\left(-2 \cos ^{2} \theta-1+4 \cos ^{4} \theta\right)-c_{20} a_{00} \cos ^{2} \theta\left(-2 \cos ^{2} \theta-1+4 \cos ^{4} \theta\right) \\
& -c_{00} a_{20} \cos ^{2} \theta\left(-2 \cos ^{2} \theta-1+4 \cos ^{4} \theta\right)+\left(-4 \cos ^{6} \theta+6 \cos ^{4} \theta-2 \cos ^{2} \theta\right) a_{11} a_{00} \\
& +\left(4 \cos ^{6} \theta-2 \cos ^{2} \theta-2 \cos ^{4} \theta\right) c_{11} c_{00}-c_{10} a_{10} \cos ^{2} \theta\left(-2 \cos ^{2} \theta-1+4 \cos ^{4} \theta\right) \\
& +\left(-4 \cos ^{6} \theta+6 \cos ^{4} \theta-2 \cos ^{2} \theta\right) a_{10} a_{01}+\left(4 \cos ^{6} \theta-2 \cos ^{2} \theta-2 \cos ^{4} \theta\right) c_{10} c_{01} \\
& +a_{10}^{2} \sin \theta \cos ^{3} \theta\left(2 \cos ^{2} \theta-1\right)-c_{01}^{2} \sin ^{3} \theta \cos \theta\left(2 \cos ^{2} \theta+1\right) \\
& +a_{01}^{2} \sin ^{3} \theta \cos \theta\left(2 \cos ^{2} \theta-1\right)-c_{10}^{2} \sin \theta \cos ^{3} \theta\left(2 \cos ^{2} \theta+1\right) \text {, } \\
& g_{2,4}(\theta)=-c_{03} a_{00} \sin ^{3} \theta\left(-2 \cos ^{2} \theta-1+4 \cos ^{4} \theta\right)-c_{00} a_{03} \sin ^{3} \theta\left(-2 \cos ^{2} \theta-1+4 \cos ^{4} \theta\right) \\
& -c_{30} a_{00} \cos ^{3} \theta\left(-2 \cos ^{2} \theta-1+4 \cos ^{4} \theta\right)-c_{00} a_{30} \cos ^{3} \theta\left(-2 \cos ^{2} \theta-1+4 \cos ^{4} \theta\right) \\
& +d_{00} \sin \theta\left(2 \cos ^{2} \theta+1\right)+b_{00} \cos \theta\left(2 \cos ^{2} \theta-1\right)-c_{20} a_{10} \cos ^{3} \theta\left(-2 \cos ^{2} \theta-1+4 \cos ^{4} \theta\right) \\
& -c_{10} a_{20} \cos ^{3} \theta\left(-2 \cos ^{2} \theta-1+46 \cos ^{4} \theta\right)-c_{02} a_{01} \sin ^{3} \theta\left(-2 \cos ^{2} \theta-1+4 \cos ^{4} \theta\right) \\
& -c_{01} a_{02} \sin ^{3} \theta\left(-2 \cos ^{2} \theta-1+4 \cos ^{4} \theta\right)-c_{21} a_{00} \cos ^{2} \theta \sin \theta\left(-2 \cos ^{2} \theta-1+4 \cos ^{4} \theta\right) \\
& -c_{00} a_{21} \cos ^{2} \theta \sin \theta\left(-2 \cos ^{2} \theta-1+4 \cos ^{4} \theta\right)-2 c_{12} c_{00} \sin ^{3} \theta \cos ^{2} \theta\left(2 \cos ^{2} \theta+1\right) \\
& -2 c_{30} c_{00} \cos ^{4} \theta \sin \theta\left(2 \cos ^{2} \theta+1\right)+2 a_{12} a_{00} \sin ^{3} \theta \cos ^{2} \theta\left(2 \cos ^{2} \theta-1\right) \\
& +2 a_{03} a_{00} \sin ^{4} \theta \cos \theta\left(2 \cos ^{2} \theta-1\right)+2 a_{30} a_{00} \cos ^{4} \theta \sin \theta\left(2 \cos ^{2} \theta-1\right) \\
& +2 a_{02} a_{01} \sin ^{4} \theta \cos \theta\left(2 \cos ^{2} \theta-1\right) \\
& -2 c_{10} c_{02} \sin ^{3} \theta \cos ^{2} \theta\left(2 \cos ^{2} \theta+1\right)-2 c_{20} c_{10} \cos ^{4} \theta \sin \theta\left(2 \cos ^{2} \theta+1\right) \\
& +2 a_{11} a_{01} \sin ^{3} \theta \cos ^{2} \theta\left(2 \cos ^{2} \theta-1\right)-c_{20} a_{01} \cos ^{2} \theta \sin \theta\left(-2 \cos ^{2} \theta-1+4 \cos ^{4} \theta\right)
\end{aligned}
$$




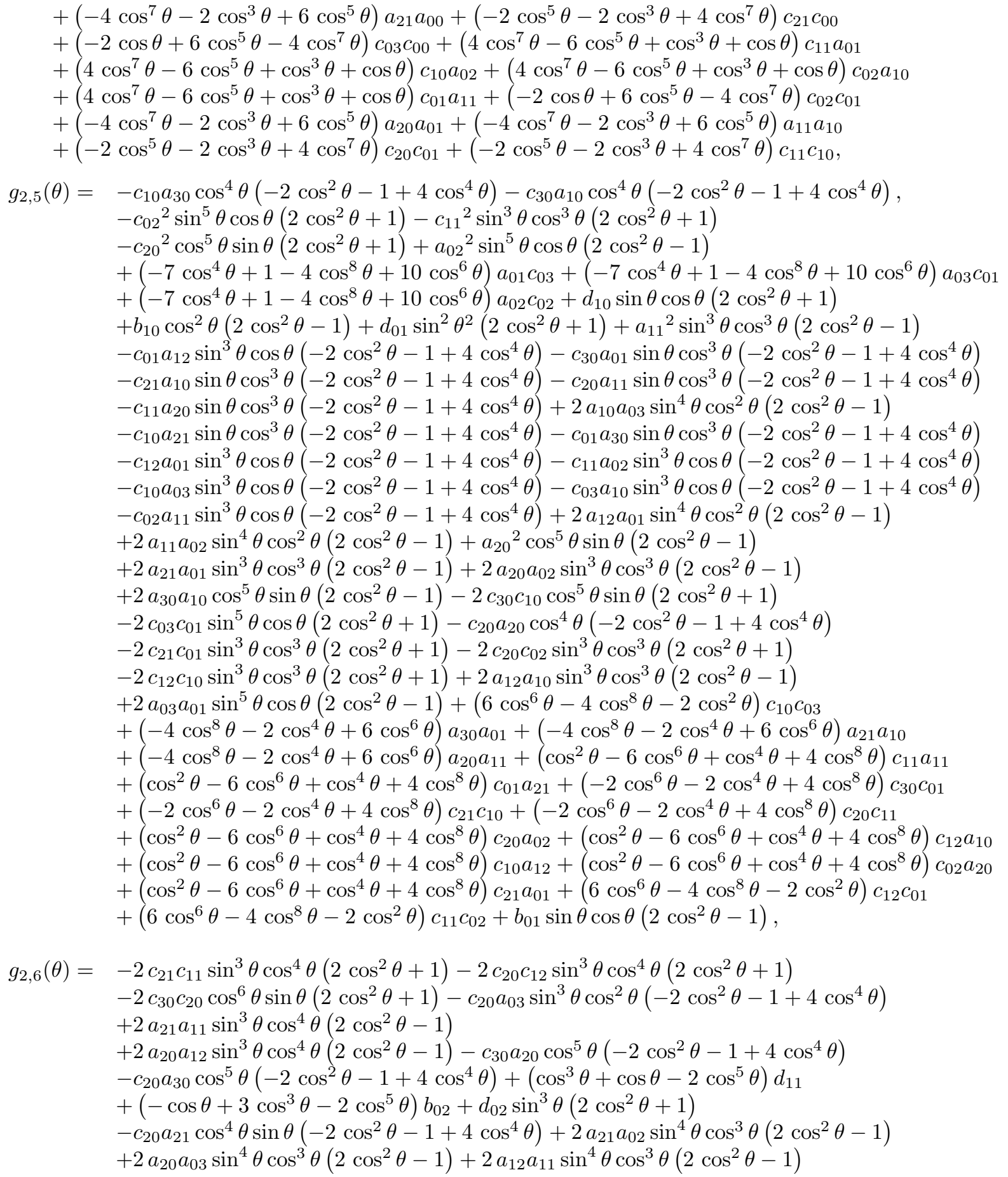




$$
\begin{aligned}
& +2 a_{03} a_{02} \sin ^{6} \theta \cos \theta\left(2 \cos ^{2} \theta-1\right)-2 c_{03} c_{02} \sin ^{6} \theta \cos \theta\left(2 \cos ^{2} \theta+1\right) \\
& +2 a_{11} a_{03} \sin ^{5} \theta \cos ^{2} \theta\left(2 \cos ^{2} \theta-1\right)-2 c_{11} c_{03} \sin ^{5} \theta \cos ^{2} \theta\left(2 \cos ^{2} \theta+1\right) \\
& -2 c_{30} c_{02} \sin ^{3} \theta \cos ^{4} \theta\left(2 \cos ^{2} \theta+1\right)+2 a_{12} a_{02} \sin ^{5} \theta \cos ^{2} \theta\left(2 \cos ^{2} \theta-1\right) \\
& -c_{11} a_{30} \cos ^{4} \theta \sin \theta\left(-2 \cos ^{2} \theta-1+4 \cos ^{4} \theta\right) \\
& -c_{11} a_{12} \sin ^{3} \theta \cos ^{2} \theta\left(-2 \cos ^{2} \theta-1+4 \cos ^{4} \theta\right)-c_{21} a_{02} \sin ^{3} \theta \cos ^{2} \theta\left(-2 \cos ^{2} \theta-1+4 \cos ^{4} \theta\right) \\
& +b_{20} \cos ^{3} \theta\left(2 \cos ^{2} \theta-1\right)-2 c_{12} c_{02} \sin ^{5} \theta \cos ^{2} \theta\left(2 \cos ^{2} \theta+1\right) \\
& -c_{12} a_{11} \sin ^{3} \theta \cos ^{2} \theta\left(-2 \cos ^{2} \theta-1+4 \cos ^{4} \theta\right)-c_{30} a_{11} \cos ^{4} \theta \sin \theta\left(-2 \cos ^{2} \theta-1+4 \cos ^{4} \theta\right) \\
& -c_{21} a_{20} \cos ^{4} \theta \sin \theta\left(-2 \cos ^{2} \theta-1+4 \cos ^{4} \theta\right)+2 a_{30} a_{20} \cos ^{6} \theta \sin \theta\left(2 \cos ^{2} \theta-1\right) \\
& -c_{03} a_{20} \sin ^{3} \theta \cos ^{2} \theta\left(-2 \cos ^{2} \theta-1+4 \cos ^{4} \theta\right)-c_{02} a_{21} \sin ^{3} \theta \cos ^{2} \theta\left(-2 \cos ^{2} \theta-1+4 \cos ^{4} \theta\right) \\
& +2 a_{30} a_{02} \sin ^{3} \theta \cos ^{4} \theta\left(2 \cos ^{2} \theta-1\right)-c_{03} a_{02} \sin ^{5} \theta\left(-2 \cos ^{2} \theta-1+4 \cos ^{4} \theta\right) \\
& +d_{20} \cos ^{2} \theta \sin \theta\left(2 \cos ^{2} \theta+1\right)+b_{11} \cos ^{2} \theta \sin \theta\left(2 \cos ^{2} \theta-1\right) \\
& -c_{02} a_{03} \sin ^{5} \theta\left(-2 \cos ^{2} \theta-1+4 \cos ^{4} \theta\right)+\left(\cos \theta+10 \cos ^{7} \theta-7 \cos ^{5} \theta-4 \cos ^{9} \theta\right) c_{11} a_{03} \\
& +\left(\cos \theta+10 \cos ^{7} \theta-7 \cos ^{5} \theta-4 \cos ^{9} \theta\right) c_{03} a_{11}+\left(\cos ^{5} \theta-6 \cos ^{7} \theta+4 \cos ^{9} \theta+\cos ^{3} \theta\right) c_{21} a_{11} \\
& +\left(\cos ^{5} \theta-6 \cos ^{7} \theta+4 \cos ^{9} \theta+\cos ^{3} \theta\right) c_{11} a_{21}+\left(\cos ^{5} \theta-6 \cos ^{7} \theta+4 \cos ^{9} \theta+\cos ^{3} \theta\right) c_{20} a_{12} \\
& +\left(\cos ^{5} \theta-6 \cos ^{7} \theta+4 \cos ^{9} \theta+\cos ^{3} \theta\right) c_{12} a_{20}+\left(\cos ^{5} \theta-6 \cos ^{7} \theta+4 \cos ^{9} \theta+\cos ^{3} \theta\right) c_{02} a_{30} \\
& +\left(\cos \theta+10 \cos ^{7} \theta-7 \cos ^{5} \theta-4 \cos ^{9} \theta\right) c_{12} a_{02}+\left(\cos \theta+10 \cos ^{7} \theta-7 \cos ^{5} \theta-4 \cos ^{9} \theta\right) c_{02} a_{12} \\
& +\left(\cos ^{5} \theta-6 \cos ^{7} \theta+4 \cos ^{9} \theta+\cos ^{3} \theta\right) c_{30} a_{02}+\left(-2 \cos ^{3} \theta+6 \cos ^{7} \theta-4 \cos ^{9} \theta\right) c_{21} c_{02} \\
& +\left(-2 \cos ^{3} \theta+6 \cos ^{7} \theta-4 \cos ^{9} \theta\right) c_{20} c_{03}+\left(-2 \cos ^{3} \theta+6 \cos ^{7} \theta-4 \cos ^{9} \theta\right) c_{12} c_{11} \\
& +\left(-4 \cos ^{9} \theta+6 \cos ^{7} \theta-2 \cos ^{5} \theta\right) a_{30} a_{11}+\left(-4 \cos ^{9} \theta+6 \cos ^{7} \theta-2 \cos ^{5} \theta\right) a_{21} a_{20} \\
& +\left(4 \cos ^{9} \theta-2 \cos ^{5} \theta-2 \cos ^{7} \theta\right) c_{30} c_{11}+\left(4 \cos ^{9} \theta-2 \cos ^{5} \theta-2 \cos ^{7} \theta\right) c_{21} c_{20}, \\
& g_{2,7}(\theta)=-c_{21}^{2} \sin ^{3} \theta \cos ^{5} \theta\left(2 \cos ^{2} \theta+1\right)+a_{12}^{2} \sin ^{5} \theta \cos ^{3} \theta\left(-1+2 \cos ^{2} \theta\right) \\
& -c_{03}{ }^{2} \sin ^{7} \theta \cos \theta\left(2 \cos ^{2} \theta+1\right)+a_{03}^{2} \sin ^{7} \theta \cos \theta\left(-1+2 \cos ^{2} \theta\right) \\
& +a_{21}{ }^{2} \sin ^{3} \theta \cos ^{5} \theta\left(-1+2 \cos ^{2} \theta\right)-c_{12}^{2} \sin ^{5} \theta \cos ^{3} \theta\left(2 \cos ^{2} \theta+1\right)-c_{30}^{2} \\
& \cos ^{7} \theta \sin \theta\left(2 \cos ^{2} \theta+1\right)+2 a_{30} a_{12} \sin ^{3} \theta \cos ^{5} \theta\left(-1+2 \cos ^{2} \theta\right) \\
& -c_{03} a_{12} \sin ^{5} \theta \cos \theta\left(4 \cos ^{4} \theta-2 \cos ^{2} \theta-1\right)-c_{30} a_{21} \cos ^{5} \theta \sin \theta\left(4 \cos ^{4} \theta-2 \cos ^{2} \theta-1\right) \\
& -c_{12} a_{21} \sin ^{3} \theta \cos ^{3} \theta\left(4 \cos ^{4} \theta-2 \cos ^{2} \theta-1\right)-c_{30} a_{03} \sin ^{3} \theta \cos ^{3} \theta\left(4 \cos ^{4} \theta-2 \cos ^{2} \theta-1\right) \\
& -2 c_{21} c_{03} \sin ^{5} \theta \cos ^{3} \theta\left(2 \cos ^{2} \theta+1\right)-2 c_{12} c_{03} \sin ^{6} \theta \cos ^{2} \theta\left(2 \cos ^{2} \theta+1\right) \\
& +2 a_{30} a_{03} \sin ^{4} \theta \cos ^{4} \theta\left(-1+2 \cos ^{2} \theta\right)+2 a_{12} a_{03}(\sin \theta)^{6} \cos ^{2} \theta\left(-1+2 \cos ^{2} \theta\right) \\
& +2 a_{21} a_{12} \sin ^{4} \theta \cos ^{4} \theta\left(-1+2 \cos ^{2} \theta\right)-c_{21} a_{30} \cos ^{5} \theta \sin \theta\left(4 \cos ^{4} \theta-2 \cos ^{2} \theta-1\right) \\
& -c_{03} a_{30} \sin ^{3} \theta \cos ^{3} \theta\left(4 \cos ^{4} \theta-2 \cos ^{2} \theta-1\right)-c_{21} a_{12} \sin ^{3} \theta \cos ^{3} \theta\left(4 \cos ^{4} \theta-2 \cos ^{2} \theta-1\right) \\
& +b_{03} \sin ^{3} \theta \cos \theta\left(-1+2 \cos ^{2} \theta\right)+b_{12} \sin ^{2} \theta \cos ^{2} \theta\left(-1+2 \cos ^{2} \theta\right) \\
& -c_{30} a_{30} \cos ^{6} \theta\left(4 \cos ^{4} \theta-2 \cos ^{2} \theta-1\right)-a_{03} c_{03} \sin ^{6} \theta\left(4 \cos ^{4} \theta-2 \cos ^{2} \theta-1\right) \\
& +d_{12} \sin ^{3} \theta \cos \theta\left(2 \cos ^{2} \theta+1\right)+d_{21} \sin ^{2} \theta \cos ^{2} \theta\left(2 \cos ^{2} \theta+1\right) \\
& +d_{30} \sin \theta \cos ^{3} \theta\left(2 \cos ^{2} \theta+1\right)-2 c_{30} c_{12} \sin ^{3} \theta \cos ^{5} \theta\left(2 \cos ^{2} \theta+1\right) \\
& +2 a_{21} a_{03} \sin ^{5} \theta \cos ^{3} \theta\left(-1+2 \cos ^{2} \theta\right)-c_{12} a_{03} \sin ^{5} \theta \cos \theta\left(4 \cos ^{4} \theta-2 \cos ^{2} \theta-1\right) \\
& +d_{03} \sin ^{4} \theta\left(2 \cos ^{2} \theta+1\right)+b_{30} \cos ^{4} \theta\left(-1+2 \cos ^{2} \theta\right)+a_{30} \cos ^{7} \theta \sin \theta\left(-1+2 \cos ^{2} \theta\right) \\
& +\left(6 \cos ^{8} \theta-4 \cos ^{10} \theta-2 \cos ^{4} \theta\right) c_{30} c_{03}+\left(6 \cos ^{8} \theta-4 \cos ^{10} \theta-2 \cos ^{4} \theta\right) c_{21} c_{12} \\
& +\left(4 \cos ^{10} \theta-2 \cos ^{8} \theta-2 \cos ^{6} \theta\right) c_{30} c_{21}+b_{21} \sin \theta \cos ^{3} \theta\left(-1+2 \cos ^{2} \theta\right) \\
& +\left(-6 \cos ^{8} \theta+\cos ^{4} \theta+\cos ^{6} \theta+4 \cos ^{10} \theta\right) c_{21} a_{21}+\left(-4 \cos ^{10} \theta-2 \cos ^{6} \theta+6 \cos ^{8} \theta\right) a_{30} a_{21} \\
& +\left(\cos ^{2} \theta-7 \cos ^{6} \theta+10 \cos ^{8} \theta-4 \cos ^{10} \theta\right) c_{03} a_{21}+\left(-6 \cos ^{8} \theta+\cos ^{4} \theta+\cos ^{6} \theta+4 \cos ^{10} \theta\right) c_{12} a_{30} \\
& +\left(\cos ^{2} \theta-7 \cos ^{6} \theta+10 \cos ^{8} \theta-4 \cos ^{10} \theta\right) c_{12} a_{12}+\left(-6 \cos ^{8} \theta+\cos ^{4} \theta+\cos ^{6} \theta+4 \cos ^{10} \theta\right) c_{30} a_{12} \\
& +\left(\cos ^{2} \theta-7 \cos ^{6} \theta+10 \cos ^{8} \theta-4 \cos ^{10} \theta\right) c_{21} a_{03} \text {. }
\end{aligned}
$$




\section{Appendix B}

We present the expressions of $A_{4}, A_{2}, A_{0}, A_{5}, A_{3}, A_{1}, \tilde{A}_{1}$ that appear in equation (9).

$$
\begin{aligned}
& A_{4}(\theta)=\mathrm{e}^{5 \sin ^{2} \theta}\left[\left[-\frac{\left(4 a_{01} c_{00} I_{2}-8 I_{1} a_{01} c_{00}-8 I_{1} c_{00} c_{10}-4 a_{00} c_{01} I_{3}+4 c_{00} c_{10} I_{2}\right) \cos ^{4} \theta}{I_{2}-2 I_{1}}\right.\right. \\
& -\frac{\left(-4 I_{1} c_{00} c_{10}+2 a_{00} c_{01} I_{3}-2 a_{01} c_{00} I_{2}+2 c_{00} c_{10} I_{2}+4 I_{1} a_{01} c_{00}\right) \cos ^{2} \theta}{I_{2}-2 I_{1}} \\
& \left.-\frac{\left(-a_{01} c_{00} I_{2}+2 I_{1} a_{01} c_{00}+2 I_{1} a_{00} c_{01}-a_{00} c_{01} I_{2}\right)}{I_{2}-2 I_{1}}\right] \sin \theta \\
& -\frac{\left(4 c_{00} c_{01} I_{3}+4 a_{00} c_{10} I_{2}-8 I_{1} a_{00} a_{01}-8 I_{1} a_{00} c_{10}+4 a_{00} a_{01} I_{2}\right)(\cos \theta)^{5}}{I_{2}-2 I_{1}} \\
& -\frac{\left(12 I_{1} a_{00} a_{01}+4 I_{1} a_{00} c_{10}-2 c_{00} c_{01} I_{3}-2 a_{00} c_{10} I_{2}-6 a_{00} a_{01} I_{2}\right)(\cos \theta)^{3}}{I_{2}-2 I_{1}} \\
& \left.-\frac{\left(-2 c_{00} c_{01} I_{1}+c_{00} c_{01} I_{2}-4 I_{1} a_{00} a_{01}+2 a_{00} a_{01} I_{2}+2 I_{1} a_{00} c_{10}-c_{00} c_{01} I_{3}-a_{00} c_{10} I_{2}\right) \cos \theta}{I_{2}-2 I_{1}}\right], \\
& A_{2}(\theta)=\left[A_{2,7} \cos ^{7} \theta+A_{2,6} \cos ^{6} \theta \sin \theta+A_{2,5} \cos ^{5} \theta+A_{2,4} \cos ^{4} \theta \sin \theta+A_{2,3} \cos ^{3} \theta+A_{2,2} \cos ^{2} \theta \sin \theta\right. \\
& \left.+A_{2,1} \cos \theta+A_{2,0} \theta \sin \theta\right] \exp \left(3 \sin ^{2} \theta\right) \\
& A_{2,7}=4 a_{03} a_{00}-4 a_{21} a_{00}-4 c_{30} a_{00}+4 c_{12} a_{00}-4 a_{20} a_{01}+4 a_{02} a_{01}+4 c_{11} a_{01}-4 c_{10} a_{20} \\
& +4 c_{10} a_{02}+4 \frac{a_{11} c_{01} I_{3}}{2 I_{1}-I_{2}}+4 c_{00} a_{12}+4 c_{03} c_{00}+8 c_{21} c_{00}+4 \frac{c_{01} c_{20} I_{3}}{2 I_{1}-I_{2}} \\
& -4 \frac{c_{01} c_{02} I_{3}}{2 I_{1}-I_{2}}+4 c_{11} c_{10} \\
& A_{2,6}=\quad-4 a_{12} a_{00}-4 c_{03} a_{00}-8 c_{21} a_{00}-4 a_{11} a_{01}-4 c_{20} a_{01}+4 c_{02} a_{01}-4 \frac{a_{20} c_{01} I_{3}}{2 I_{1}-I_{2}} \\
& +4 \frac{a_{02} c_{01} I_{3}}{2 I_{1}-I_{2}}-4 a_{11} c_{10}+4 a_{03} c_{00}-4 a_{21} c_{00}-4 c_{30} c_{00}+4 c_{12} c_{00} \\
& +4 \frac{c_{01} c_{11} I_{3}}{2 I_{1}-I_{2}}-4 c_{20} c_{10}+4 c_{10} c_{02} \text {, } \\
& A_{2,5}=\quad-10 a_{03} a_{00}+6 a_{21} a_{00}+2 c_{30} a_{00}-6 c_{12} a_{00}+6 a_{20} a_{01}-10 a_{02} a_{01}-6 c_{11} a_{01}+2 c_{10} a_{20} \\
& -6 c_{10} a_{02}-6 \frac{a_{11} c_{01} I_{3}}{2 I_{1}-I_{2}}-6 c_{00} a_{12}+2 c_{03} c_{00}-4 c_{21} c_{00}-2 \frac{c_{01} c_{20} I_{3}}{2 I_{1}-I_{2}} \\
& +6 \frac{c_{01} c_{02} I_{3}}{2 I_{1}-I_{2}}-2 c_{11} c_{10}, \\
& A_{2,4}=\quad 6 a_{00} a_{12}-2 a_{00} c_{03}+4 a_{00} c_{21}+6 a_{01} a_{11}+2 a_{01} c_{20}-6 a_{01} c_{02}+2 \frac{a_{20} c_{01} I_{3}}{2 I_{1}-I_{2}} \\
& -6 \frac{a_{02} c_{01} I_{3}}{2 I_{1}-I_{2}}+2 a_{11} c_{10}-6 a_{03} c_{00}+2 a_{21} c_{00}-2 c_{00} c_{30}-2 c_{00} c_{12}-2 \frac{c_{01} c_{11} I_{3}}{2 I_{1}-I_{2}} \\
& -2 c_{10} c_{20}-2 c_{02} c_{10} \text {, } \\
& A_{2,3}=2 b_{00}+8 a_{00} a_{03}-2 a_{00} a_{21}+a_{00} c_{30}+a_{00} c_{12}-2 a_{01} a_{20}+8 a_{01} a_{02}+a_{01} c_{11}+a_{20} c_{10} \\
& +a_{02} c_{10}-\frac{\left(-2 I_{3}-I_{2}+2 I_{1}\right) c_{01} a_{11}}{2 I_{1}-I_{2}}+a_{12} c_{00}-2 c_{00} c_{03}-3 c_{00} c_{21} \\
& -\frac{\left(-I_{2}+2 I_{1}+I_{3}\right) c_{01} c_{20}}{2 I_{1}-I_{2}}+\frac{\left(2 I_{1}-I_{3}-I_{2}\right) c_{02} c_{01}}{-I_{2}+2 I_{1}}-2 c_{10} c_{11},
\end{aligned}
$$




$$
\begin{aligned}
A_{2,2}= & 2 d_{00}-2 a_{00} a_{12}+a_{00} c_{03}+a_{00} c_{21}-2 a_{01} a_{11}+a_{01} c_{20}+a_{01} c_{02}+a_{20} c_{01} \\
& -\frac{\left(-2 I_{3}-I_{2}+2 I_{1}\right) c_{01} a_{02}}{2 I_{1}-I_{2}}+a_{11} c_{10}+a_{03} c_{00}+a_{21} c_{00}-2 c_{00} c_{12} \\
& -\frac{\left(-I_{2}+2 I_{1}+I_{3}\right) c_{11} c_{01}}{2 I_{1}-I_{2}}-2 c_{02} c_{10}, \\
A_{2,1}= & -b_{00}-2 a_{00} a_{03}+a_{00} c_{12}-2 a_{01} a_{02}+a_{01} c_{11}+a_{02} c_{10}+a_{11} c_{01}+a_{12} c_{00}-2 c_{00} c_{03} \\
& -\frac{\left(-I_{2}+2 I_{1}+I_{3}\right) c_{01} c_{02}}{2 I_{1}-I_{2}}, \\
A_{2,0}= & a_{03} c_{00}+d_{00}+a_{00} c_{03}+a_{01} c_{02}+a_{02} c_{01} .
\end{aligned}
$$

Now we have

$$
\begin{aligned}
& A_{0}(\theta)=A_{0,9} \cos ^{9} \theta+A_{0,8} \cos ^{8} \theta+A_{0,7} \cos ^{7} \theta+A_{0,6} \cos ^{6} \theta+A_{0,5} \cos ^{5} \theta+A_{0,4} \cos ^{4} \theta \\
& +A_{0,3} \cos ^{3} \theta+A_{0,2} \cos ^{2} \theta+A_{0,1} \cos \theta+A_{0,0},
\end{aligned}
$$

with

$$
\begin{aligned}
& A_{0,9}(\theta)=-\left(-4 a_{21} c_{11}+4 c_{02} c_{03}+8 c_{02} c_{21}-4 c_{03} c_{20}+4 c_{11} c_{12}-4 c_{11} c_{30}-8 c_{20} c_{21}\right. \\
& -8 a_{11} c_{21}+4 a_{12} c_{02}-4 a_{12} c_{20}+4 a_{20} a_{21}-4 a_{20} c_{12}+4 a_{20} c_{30}+4 a_{02} a_{03}-4 a_{02} a_{21} \\
& \left.+4 a_{02} c_{12}-4 a_{02} c_{30}-4 a_{03} a_{20}+4 a_{03} c_{11}-4 a_{11} a_{12}-4 a_{11} c_{03}\right) \mathrm{e}^{\sin ^{2} \theta}, \\
& A_{0,8}(\theta)=-\left(-4 a_{03} c_{20}+4 a_{11} a_{21}-4 a_{11} c_{12}+4 a_{12} a_{20}-4 a_{02} c_{03}-8 a_{02} c_{21}-4 a_{03} a_{11}\right. \\
& +4 a_{03} c_{02}-8 c_{11} c_{21}-4 c_{12} c_{20}+4 c_{20} c_{30}+4 a_{11} c_{30}-4 a_{02} a_{12}-4 a_{12} c_{11} \\
& \left.+4 a_{20} c_{03}+8 a_{20} c_{21}-4 a_{21} c_{02}+4 a_{21} c_{20}+4 c_{02} c_{12}-4 c_{02} c_{30}-4 c_{03} c_{11}\right) \mathrm{e}^{\sin ^{2} \theta} \sin \theta \text {, } \\
& A_{0,7}(\theta)=-\left(2 c_{11} c_{30}+4 c_{20} c_{21}-14 a_{02} a_{03}+10 a_{02} a_{21}-10 a_{02} c_{12}+6 a_{02} c_{30}+10 a_{03} a_{20}\right. \\
& -10 a_{03} c_{11}+10 a_{11} a_{12}+2 a_{11} c_{03}+12 a_{11} c_{21}-10 a_{12} c_{02}+6 a_{12} c_{20}-6 a_{20} a_{21}+6 a_{20} c_{12} \\
& \left.-2 a_{20} c_{30}+6 a_{21} c_{11}-2 c_{02} c_{03}-12 c_{02} c_{21}-2 c_{03} c_{20}-6 c_{11} c_{12}\right) \mathrm{e}^{\sin ^{2} \theta} \text {, } \\
& A_{0,6}(\theta)=-\left(2 c_{20} c_{30}+2 a_{02} c_{03}+6 a_{12} c_{11}+2 a_{20} c_{03}-4 a_{20} c_{21}+6 a_{21} c_{02}-2 a_{21} c_{20}-6 c_{02} c_{12}\right. \\
& +2 c_{02} c_{30}-2 c_{03} c_{11}+4 c_{11} c_{21}+2 c_{12} c_{20}-6 a_{11} a_{21}+6 a_{11} c_{12}-2 a_{11} c_{30}-6 a_{12} a_{20} \\
& \left.+6 a_{03} c_{20}+12 a_{02} c_{21}+10 a_{03} a_{11}+10 a_{02} a_{12}-10 a_{03} c_{02}\right) \mathrm{e}^{\sin ^{2} \theta} \sin \theta \text {, } \\
& A_{0,5}(\theta)=-\left[-2 b_{20}+18 a_{02} a_{03}-8 a_{11} a_{12}+2 b_{02}-a_{20} c_{12}-a_{20} c_{30}-a_{21} c_{11}-4 c_{02} c_{03}+c_{02} c_{21}\right. \\
& +2 c_{03} c_{20}+2 c_{1,1} c_{30}+3 c_{20} c_{21}+3 a_{11} c_{03}-3 a_{11} c_{21}+7 a_{12} c_{02}-a_{12} c_{20}+2 a_{20} a_{21} \\
& \left.+7 a_{02} c_{12}-a_{02} c_{30}+2 d_{11}-8 a_{02} a_{21}-8 a_{03} a_{20}+7 a_{03} c_{11}\right] \mathrm{e}^{\sin ^{2} \theta}, \\
& A_{0,4}(\theta)=-\left[7 a_{03} c_{02}-a_{03} c_{20}-8 a_{03} a_{11}-a_{11} c_{30}+2 a_{12} a_{20}-a_{12} c_{11}-a_{11} c_{12}-a_{20} c_{21}\right. \\
& -8 a_{02} a_{12}+3 a_{02} c_{0,3}-3 a_{02} c_{21}+2 c_{03} c_{11}+3 c_{11} c_{21}+2 c_{12} c_{20}+2 a_{11} a_{21}-a_{20} c_{03} \\
& \left.-2 b_{11}+2 d_{02}-2 d_{2,0}+2 c_{02} c_{30}-a_{21} c_{02}-a_{21} c_{20}\right) \mathrm{e}^{\sin ^{2} \theta} \sin \theta \text {, } \\
& A_{0,3}(\theta)=-\left[-a_{11} c_{21}-d_{11}-a_{12} c_{20}-3 b_{02}+b_{20}+2 a_{11} a_{12}+3 c_{02} c_{21}+2 c_{03} c_{20}-a_{20} c_{12}\right. \\
& \left.-a_{21} c_{11}-10 a_{02} a_{03}+2 a_{02} a_{21}+2 c_{11} c_{12}+2 a_{03} a_{20}-a_{02} c_{30}\right] \mathrm{e}^{\sin ^{2} \theta}, \\
& A_{0,2}(\theta)=-\left[2 a_{03} a_{11}-a_{03} c_{20}-a_{02} c_{21}-a_{20} c_{03}-a_{21} c_{02}-a_{11} c_{12}-a_{12} c_{11}-d_{02}-d_{20}\right. \\
& \left.+2 c_{02} c_{12}+2 c_{03} c_{11}+b_{11}+2 a_{02} a_{12}\right] \mathrm{e}^{\sin ^{2} \theta} \sin \theta, \\
& A_{0,1}(\theta)=-\left(2 c_{02} c_{03}-a_{02} c_{12}+b_{02}-d_{11}+2 a_{02} a_{03}-a_{03} c_{11}-a_{11} c_{03}-a_{12} c_{02}\right) \mathrm{e}^{\sin ^{2} \theta}, \\
& A_{0,0}(\theta)=-\left[-d_{02}-a_{02} c_{03}-a_{03} c_{02}\right] \mathrm{e}^{\sin ^{2} \theta} \sin \theta
\end{aligned}
$$


Additionally, we have

$$
\begin{aligned}
& A_{5}(\theta)=\left[\sin \theta \cos \theta\left(-1+2 \cos ^{2} \theta\right) a_{00}^{2}+\left(-4 \cos ^{4} \theta+2 \cos ^{2} \theta+1\right) c_{00} a_{00}\right. \\
& \left.-\sin \theta \cos \theta\left(2 \cos ^{2} \theta+1\right) c_{00}^{2}\right] \mathrm{e}^{6 \sin ^{2} \theta} \text {, } \\
& A_{3}(\theta)=2 \cos ^{3} \theta \sin \theta\left(-1+2 \cos ^{2} \theta\right) a_{00} a_{20}-2 \cos \theta \sin \theta\left(\cos ^{2} \theta-1\right)\left(-1+2 \cos ^{2} \theta\right) a_{00} a_{02} \\
& -2 \cos ^{2} \theta\left(\cos ^{2} \theta-1\right)\left(-1+2 \cos ^{2} \theta\right) a_{00} a_{11}-\cos ^{2} \theta\left(4 \cos ^{4} \theta-1-2 \cos ^{2} \theta\right) a_{00} c_{20} \\
& +\left(\cos ^{2} \theta-1\right)\left(4 \cos ^{4} \theta-1-2 \cos ^{2} \theta\right) a_{00} c_{02}-\cos \theta \sin \theta\left(4 \cos ^{4} \theta-1-2 \cos ^{2} \theta\right) c_{11} a_{00} \\
& -\cos \theta \sin \theta\left(\cos ^{2} \theta-1\right)\left(-1+2 \cos ^{2} \theta\right) a_{01}^{2}-\left(\cos ^{2} \theta-1\right) \frac{2 I_{1}-4 \cos ^{4} \theta I_{3}+2 \cos ^{2} \theta I_{3}-I_{2}}{2 I_{1}-I_{2}} a_{01} c_{01} \\
& -\cos \theta \sin \theta\left(4 \cos ^{4} \theta-1-2 \cos ^{2} \theta\right) c_{10} a_{01}-\cos ^{2} \theta\left(4 \cos ^{4} \theta-1-2 \cos ^{2} \theta\right) c_{00} a_{20} \\
& +\left(\cos ^{2} \theta-1\right)\left(4 \cos ^{4} \theta-1-2 \cos ^{2} \theta\right) c_{00} a_{02}-\cos \theta \sin \theta\left(4 \cos ^{4} \theta-1-2 \cos ^{2} \theta\right) c_{00} a_{11} \\
& -2 \cos ^{3} \theta \sin \theta\left(2 \cos ^{2} \theta+1\right) c_{00} c_{20}+2 \cos \theta \sin \theta\left(\cos ^{2} \theta-1\right)\left(2 \cos ^{2} \theta+1\right) c_{00} c_{02} \\
& +2 \cos ^{2} \theta\left(\cos ^{2} \theta-1\right)\left(2 \cos ^{2} \theta+1\right) c_{11} c_{00}-\cos \theta \sin \theta I_{3} \frac{2 I_{1}-I_{2}+\cos ^{2} \theta I_{3}-2 \cos ^{4} \theta I_{3}}{2 I_{1}-I_{2}} c_{01}{ }^{2} \\
& \left.-\cos ^{2} \theta \frac{2 I_{1}+I_{3}+2 \cos ^{2} \theta I_{3}-I_{2}-4 \cos ^{4} \theta I_{3}}{2 I_{1}-I_{2}} c_{10} c_{01}-\cos ^{3} \theta \sin \theta\left(2 \cos ^{2} \theta+1\right) c_{10}^{2}\right] \exp \left(4 \sin ^{2} \theta\right) \text {, } \\
& A_{1}(\theta)=\left[-\cos ^{4} \theta \frac{2 I_{1}+I_{3}+2 \cos ^{2} \theta I_{3}-I_{2}-4 \cos ^{4} \theta I_{3}}{2 I_{1}-I_{2}} c_{01} c_{30}+\cos ^{4} \theta\left(8 \cos ^{4} \theta-3-4 \cos ^{2} \theta\right) c_{10} c_{21}\right. \\
& +2 \cos ^{2} \theta\left(2 \cos ^{2} \theta-1\right)\left(\cos ^{2} \theta-1\right)^{2} a_{02} a_{11}+\cos ^{2} \theta\left(\cos ^{2} \theta-1\right)\left(4 \cos ^{4} \theta-1-2 \cos ^{2} \theta\right) a_{20} c_{02} \\
& +\sin \theta \cos \theta\left(2 \cos ^{2} \theta-1\right)\left(\cos ^{2} \theta-1\right)^{2} a_{02}{ }^{2}-\sin \theta \cos ^{3} \theta\left(\cos ^{2} \theta-1\right)\left(2 \cos ^{2} \theta-1\right) a_{11}{ }^{2} \\
& -2 \cos ^{4} \theta\left(\cos ^{2} \theta-1\right)\left(2 \cos ^{2} \theta-1\right) a_{11} a_{20} \\
& +\mathrm{e}^{2} \sin ^{2} \theta \cos ^{2} \theta\left(\cos ^{2} \theta-1\right)\left(8 \cos ^{4} \theta-4 \cos ^{2} \theta-1\right) a_{01} c_{21} \\
& +2 \cos ^{2} \theta\left(2 \cos ^{2} \theta-1\right)\left(\cos ^{2} \theta-1\right)^{2} a_{01} a_{12}+\cos ^{2} \theta\left(2 \cos ^{2} \theta-1\right) b_{10} \\
& +\cos ^{2} \theta\left(\cos ^{2} \theta-1\right)\left(4 \cos ^{4} \theta-1-2 \cos ^{2} \theta\right) c_{10} a_{12}+2 \cos ^{4} \theta\left(\cos ^{2} \theta-1\right)\left(2 \cos ^{2} \theta+1\right) c_{11} c_{20} \\
& -\sin \theta \cos \theta\left(2 \cos ^{2} \theta+1\right)\left(\cos ^{2} \theta-1\right)^{2} c_{02}^{2}-2 \cos ^{2} \theta\left(2 \cos ^{2} \theta+1\right)\left(\cos ^{2} \theta-1\right)^{2} c_{11} c_{02} \\
& +\sin \theta \cos ^{3} \theta\left(\cos ^{2} \theta-1\right)\left(2 \cos ^{2} \theta+1\right) c_{11}^{2}+\cos ^{2} \theta\left(\cos ^{2} \theta-1\right)\left(4 \cos ^{4} \theta-1-2 \cos ^{2} \theta\right) c_{11} a_{11} \\
& +\cos ^{2} \theta\left(\cos ^{2} \theta-1\right)\left(4 \cos ^{4} \theta-1-2 \cos ^{2} \theta\right) a_{02} c_{20} \\
& -\sin \theta \cos \theta\left(\cos ^{2} \theta-1\right) \frac{2 I_{1}-4 \cos ^{4} \theta I_{3}+2 \cos ^{2} \theta I_{3}-I_{2}}{2 I_{1}-I_{2}} a_{12} c_{01} \\
& -\sin \theta \cos \theta \frac{-4 \cos ^{6} \theta I_{3}-I_{2}+2 I_{1}+2 \cos ^{2} \theta I_{1}+\cos ^{2} \theta I_{3}+I_{3}-2 \cos ^{4} \theta I_{3}-\cos ^{2} \theta I_{2}}{2 I_{1}-I_{2}} c_{01} c_{03} \\
& -\sin \theta \cos ^{5} \theta\left(2 \cos ^{2} \theta+1\right) c_{20}^{2}-\sin \theta \cos ^{3} \theta \frac{4 \cos ^{2} \theta I_{3}+4 I_{1}-8 \cos ^{4} \theta I_{3}+I_{3}-2 I_{2}}{2 I_{1}-I_{2}} c_{01} c_{21} \\
& +2 \cos ^{2} \theta\left(\cos ^{4} \theta-\cos ^{2} \theta-1+2 \cos ^{6} \theta\right) c_{03} c_{10}+\sin \theta \cos ^{5} \theta\left(-1+2 \cos ^{2} \theta\right) a_{20}{ }^{2} \\
& -\cos ^{4} \theta\left(4 \cos ^{4} \theta-1-2 \cos ^{2} \theta\right) a_{20} c_{20}+\left(\cos ^{2} \theta-1\right)^{2} \frac{2 I_{1}-4 \cos ^{4} \theta I_{3}+2 \cos ^{2} \theta I_{3}-I_{2}}{2 I_{1}-I_{2}} a_{03} c_{01} \\
& -\left(\cos ^{2} \theta-1\right)\left(2 \cos ^{2} \theta+1\right) d_{01}+\sin \theta \cos \theta\left(2 \cos ^{2} \theta+1\right) d_{10}+\sin \theta \cos \theta\left(-1+2 \cos ^{2} \theta\right) b_{01} \\
& -\sin \theta \cos ^{3} \theta\left(4 \cos ^{4} \theta-1-2 \cos ^{2} \theta\right) a_{11} c_{20}-\sin \theta \cos ^{3} \theta\left(4 \cos ^{4} \theta-1-2 \cos ^{2} \theta\right) a_{01} c_{30} \\
& +\left(\cos ^{2} \theta-1\right)\left(4 \cos ^{6} \theta+2 \cos ^{4} \theta-(\cos \theta)^{2}-1\right) a_{01} c_{03} \\
& -\cos ^{2} \theta\left(\cos ^{2} \theta-1\right) \frac{2 I_{1}-4 \cos ^{4} \theta I_{3}+2 \cos ^{2} \theta I_{3}-I_{2}}{2 I_{1}-I_{2}} a_{21} c_{01} \\
& -\left(4 \cos ^{4} \theta-1-2 \cos ^{2} \theta\right)\left(\cos ^{2} \theta-1\right)^{2} a_{02} c_{02}-\sin \theta \cos ^{3} \theta\left(4 \cos ^{4} \theta-1-2 \cos ^{2} \theta\right) c_{11} a_{20} \\
& +\cos ^{2} \theta\left(\cos ^{2} \theta-1\right) \frac{2 I_{1}+I_{3}+2 \cos ^{2} \theta I_{3}-I_{2}-4 \cos ^{4} \theta I_{3}}{2 I_{1}-I_{2}} c_{12} c_{01} \\
& -\sin \theta \cos ^{3} \theta\left(4 \cos ^{4} \theta-1-2 \cos ^{2} \theta\right) c_{10} a_{21}-2 \sin \theta \cos ^{5} \theta\left(2 \cos ^{2} \theta+1\right) c_{10} c_{30} \\
& +\sin \theta \cos \theta\left(\cos ^{2} \theta-1\right)\left(4 \cos ^{4} \theta-1-2 \cos ^{2} \theta\right) c_{12} a_{01} \\
& -2 \sin \theta \cos ^{3} \theta\left(\cos ^{2} \theta-1\right)\left(2 \cos ^{2} \theta-1\right) a_{01} a_{21} \\
& +2 \sin \theta \cos \theta\left(-1+2 \cos ^{2} \theta\right)\left(\cos ^{2} \theta-1\right)^{2} a_{01} a_{03}-2 \sin \theta \cos ^{3} \theta\left(\cos ^{2} \theta-1\right)\left(2 \cos ^{2} \theta-1\right) a_{02} a_{20} \\
& +\sin \theta \cos \theta\left(\cos ^{2} \theta-1\right)\left(4 \cos ^{4} \theta-1-2 \cos ^{2} \theta\right) c_{11} a_{02} \\
& +\sin \theta \cos \theta\left(\cos ^{2} \theta-1\right)\left(4 \cos ^{4} \theta-1-2 \cos ^{2} \theta\right) a_{11} c_{02} \\
& +\sin \theta \cos \theta\left(\cos ^{2} \theta-1\right)\left(4 \cos ^{4} \theta-1-2 \cos ^{2} \theta\right) c_{10} a_{03} \\
& +2 \sin \theta \cos ^{3} \theta\left(\cos ^{2} \theta-1\right)\left(2 \cos ^{2} \theta+1\right) c_{12} c_{10} \\
& \left.+2 \sin \theta \cos ^{3} \theta\left(\cos ^{2} \theta-1\right)\left(2 \cos ^{2} \theta+1\right) c_{02} c_{20}\right] \mathrm{e}^{2 \sin ^{2} \theta} \text {, }
\end{aligned}
$$




$$
\begin{aligned}
& \tilde{A}_{1}(\theta)=\cos \theta \sin \theta\left(\cos ^{4} \theta-\cos ^{2} \theta+2 \cos ^{8} \theta+3 \cos ^{6} \theta-1\right) c_{03}^{2} \\
& -\left(4 \cos ^{6} \theta+2 \cos ^{4} \theta-\cos ^{2} \theta-1\right)\left(\cos ^{2} \theta-1\right)^{2} a_{03} c_{03} \\
& -\cos ^{5} \theta \sin \theta\left(4 \cos ^{4} \theta-1-2 \cos ^{2} \theta\right) a_{21} c_{30}+\cos ^{6} \theta\left(8 \cos ^{4} \theta-3-4 \cos ^{2} \theta\right) c_{21} c_{30} \\
& +2 \cos ^{4} \theta\left(\cos ^{4} \theta-\cos ^{2} \theta-1+2 \cos ^{6} \theta\right) c_{03} c_{30} \\
& +\cos ^{3} \theta \sin \theta\left(2 \cos ^{2} \theta+1\right) d_{30}+\cos ^{3} \theta \sin \theta\left(-1+2 \cos ^{2} \theta\right) b_{21} \\
& +2 c_{21}^{2} \cos ^{5} \theta \sin \theta\left(4 \cos ^{4} \theta-1-2 \cos ^{2} \theta\right)+a_{12}{ }^{2} \sin ^{5} \theta \cos ^{3} \theta\left(-1+2 \cos ^{2} \theta\right) \\
& -\cos ^{7} \theta \sin \theta\left(2 \cos ^{2} \theta+1\right) c_{30}^{2}+\left(2 \cos ^{2} \theta+1\right)\left(\cos ^{2} \theta-1\right)^{2} d_{03} \\
& +\cos ^{3} \theta \sin \theta\left(4 \cos ^{4} \theta+8 \cos ^{6} \theta-3-3 \cos ^{2} \theta\right) c_{03} c_{21}-\cos ^{2} \theta\left(\cos ^{2} \theta-1\right)\left(2 \cos ^{2} \theta+1\right) d_{21} \\
& -\cos ^{2} \theta\left(\cos ^{2} \theta-1\right)\left(-1+2 \cos ^{2} \theta\right) b_{12}+\cos ^{4} \theta\left(-1+2 \cos ^{2} \theta\right) b_{30} \\
& -\cos \theta \sin \theta\left(-1+2 \cos ^{2} \theta\right)\left(\cos ^{2} \theta-1\right)^{3} a_{03}^{2}+\cos ^{4} \theta\left(\cos ^{2} \theta-1\right)\left(4 \cos ^{4} \theta-1-2 \cos ^{2} \theta\right) a_{12} c_{30} \\
& -2 \cos ^{2} \theta\left(\cos ^{2} \theta-1\right)\left(\cos ^{4} \theta-\cos ^{2} \theta-1+2 \cos ^{6} \theta\right) c_{03} c_{12} \\
& -\cos \theta \sin \theta\left(\cos ^{2} \theta-1\right)\left(2 \cos ^{2} \theta+1\right) d_{12} \\
& -\cos \theta \sin \theta\left(\cos ^{2} \theta-1\right)\left(-1+2 \cos ^{2} \theta\right) b_{03}+2 \cos ^{4} \theta\left(-1+2 \cos ^{2} \theta\right)\left(\cos ^{2} \theta-1\right)^{2} a_{12} a_{21} \\
& +\cos ^{4} \theta\left(\cos ^{2} \theta-1\right)\left(8 \cos ^{4} \theta-4 \cos ^{2} \theta-1\right) a_{21} c_{21} \\
& -\cos ^{4} \theta\left(\cos ^{2} \theta-1\right)\left(8 \cos ^{4} \theta-3-4 \cos ^{2} \theta\right) c_{12} c_{21} \\
& -2 \cos ^{2} \theta\left(-1+2 \cos ^{2} \theta\right)\left(\cos ^{2} \theta-1\right)^{3} a_{03} a_{12}-\cos ^{2} \theta\left(8 \cos ^{4} \theta-4 \cos ^{2} \theta-1\right)\left(\cos ^{2} \theta-1\right)^{2} a_{03} c_{21} \\
& -\cos ^{2} \theta\left(4 \cos ^{4} \theta-1-2 \cos ^{2} \theta\right)\left(\cos ^{2} \theta-1\right)^{2} a_{12} c_{12} \\
& +\cos ^{2} \theta\left(\cos ^{2} \theta-1\right)\left(4 \cos ^{6} \theta+2 \cos ^{4} \theta-\cos ^{2} \theta-1\right) a_{21} c_{03} \\
& -\cos ^{5} \theta \sin \theta\left(\cos ^{2} \theta-1\right)\left(-1+2 \cos ^{2} \theta\right) a_{21}^{2} \\
& -\cos ^{3} \theta \sin \theta\left(2 \cos ^{2} \theta+1\right)\left(\cos ^{2} \theta-1\right)^{2} c_{12}^{2}+2 \cos ^{5} \theta \sin \theta\left(\cos ^{2} \theta-1\right)\left(2 \cos ^{2} \theta+1\right) c_{12} c_{30} \\
& +2 \cos ^{3} \theta \sin \theta\left(-1+2 \cos ^{2} \theta\right)\left(\cos ^{2} \theta-1\right)^{2} a_{03} a_{21} \\
& +\cos ^{3} \theta \sin \theta\left(\cos ^{2} \theta-1\right)\left(4 \cos ^{4} \theta-1-2 \cos ^{2} \theta\right) a_{03} c_{30} \\
& +\cos ^{3} \theta \sin \theta\left(\cos ^{2} \theta-1\right)\left(8 \cos ^{4} \theta-4 \cos ^{2} \theta-1\right) a_{12} c_{21} \\
& +\cos ^{3} \theta \sin \theta\left(\cos ^{2} \theta-1\right)\left(4 \cos ^{4} \theta-1-2 \cos ^{2} \theta\right) a_{21} c_{12} \\
& -\cos \theta \sin \theta\left(4 \cos ^{4} \theta-1-2 \cos ^{2} \theta\right)\left(\cos ^{2} \theta-1\right)^{2} a_{03} c_{12} \\
& +\cos \theta \sin \theta\left(\cos ^{2} \theta-1\right)\left(4 \cos ^{6} \theta+2 \cos ^{4} \theta-\cos ^{2} \theta-1\right) a_{12} c_{03} \text {. }
\end{aligned}
$$

\section{Appendix $\mathrm{C}$}

Here we present the explicit expressions of $s_{5}(\theta), s_{4}(\theta), s_{3}(\theta), s_{2}(\theta), s_{1}(\theta), \tilde{s}_{1}(\theta)$ that appear in relation (10). Thus $s_{5}(\theta)=s_{5,1}(\theta) c_{00}^{2}+s_{5,2}(\theta) a_{00}^{2}+s_{5,3}(\theta) a_{00} c_{00}$, with

$$
\begin{aligned}
s_{5,1}(\theta)= & -2 \mathrm{e}^{3 \sin ^{2} \theta}\left(\int_{0}^{\theta} \mathrm{e}^{3 \sin ^{2} w} \sin w d w+2 \int_{0}^{\theta} \mathrm{e}^{3 \sin ^{2} w} \sin w \cos ^{2} w d w\right) \\
& \left(\sin \theta+2 \sin \theta \cos ^{2} \theta\right), \\
s_{5,2}(\theta)= & -2 \mathrm{e}^{3 \sin ^{2} \theta}\left(-\int_{0}^{\theta} \mathrm{e}^{3 \sin ^{2} w} \cos w d w+2 \int_{0}^{\theta} \mathrm{e}^{3 \sin ^{2} w} \cos ^{3} w d w\right) \\
& \left(-\cos \theta+2 \cos ^{3} \theta\right),
\end{aligned}
$$




$$
\begin{aligned}
& s_{5,3}(\theta)=-2 \mathrm{e}^{3 \sin ^{2} \theta}\left(\int_{0}^{\theta} \mathrm{e}^{3 \sin ^{2} w} \sin w d w+2 \int_{0}^{\theta} \mathrm{e}^{3 \sin ^{2} w} \sin w \cos ^{2} w d w\right) \\
&\left(-\cos \theta+2 \cos ^{3} \theta\right) \\
&-2 \mathrm{e}^{3 \sin ^{2} \theta}\left(-\int_{0}^{\theta} \mathrm{e}^{3 \sin ^{2} w} \cos w d w+2 \int_{0}^{\theta} \mathrm{e}^{3 \sin ^{2} w} \cos ^{3} w d w\right) \\
&\left(\sin \theta+2 \sin \theta \cos ^{2} \theta\right) .
\end{aligned}
$$

Additionally, we have

$s_{4}(\theta)=s_{4,1}(\theta) a_{00} c_{10}-s_{4,2}(\theta) a_{00} a_{01}-s_{4,3}(\theta) c_{00} c_{01}-s_{4,4}(\theta) c_{00} c_{10}-s_{4,5}(\theta) a_{00} c_{01}-s_{4,6}(\theta) a_{01} c_{00}$, and $s_{4, i}(\theta)$ for $i=1 \cdots, 6$ are the following:

$$
\begin{aligned}
& s_{4,1}(\theta)=-\left(-\sin \theta \mathrm{e}^{-\sin ^{2} \theta} \int_{0}^{\theta} \mathrm{e}^{3 \sin ^{2} w} \cos w d w+4 \cos ^{2} \theta \int_{0}^{\theta} \mathrm{e}^{2 \sin ^{2} w} \sin w \cos w d w\right. \\
& +2 \sin \theta \mathrm{e}^{-\sin ^{2} \theta} \int_{0}^{\theta} \mathrm{e}^{3 \sin ^{2} w} \cos ^{3} w d w+4 \cos ^{2} \theta \sin \theta \mathrm{e}^{-\sin ^{2} \theta} \int_{0}^{\theta} \mathrm{e}^{3 \sin ^{2} w} \cos ^{3} w d w \\
& +8 \cos ^{2} \theta \int_{0}^{\theta} \mathrm{e}^{2 \sin ^{2} w} \cos ^{3} w \sin w d w-2 \cos ^{2} \theta \sin \theta \mathrm{e}^{-\sin ^{2} \theta} \int_{0}^{\theta} \mathrm{e}^{3 \sin ^{2} w} \cos w d w \\
& \left.-4 \int_{0}^{\theta} \mathrm{e}^{2 \sin ^{2} w} \cos ^{3} w \sin w d w-2 \int_{0}^{\theta} \mathrm{e}^{2 \sin ^{2} w} \sin w \cos w d w\right) \mathrm{e}^{3 \sin ^{2} \theta} \cos \theta, \\
& s_{4,2}(\theta)=-\left(\sin \theta \mathrm{e}^{-\sin ^{2} \theta} \int_{0}^{\theta} \mathrm{e}^{3 \sin ^{2} w} \cos w d w-2 \sin \theta \mathrm{e}^{-\sin ^{2} \theta} \int_{0}^{\theta} \mathrm{e}^{3 \sin ^{2} w} \cos ^{3} w d w\right. \\
& -4 \cos ^{2} \theta \int_{0}^{\theta} \mathrm{e}^{2 \sin ^{2} w} \sin w \cos w d w-4 \int_{0}^{\theta} \mathrm{e}^{2 \sin ^{2} w} \cos ^{3} w \sin w d w \\
& -2 \cos ^{2} \theta \sin \theta \mathrm{e}^{-\sin ^{2} \theta} \int_{0}^{\theta} \mathrm{e}^{3 \sin ^{2} w} \cos w d w \\
& +4 \cos ^{2} \theta \sin \theta \mathrm{e}^{-\sin ^{2} \theta} \int_{0}^{\theta} \mathrm{e}^{3 \sin ^{2} w} \cos ^{3} w d w+2 \int_{0}^{\theta} \mathrm{e}^{2 \sin ^{2} w} \sin w \cos w d w \\
& \left.+8 \cos ^{2} \theta \int_{0}^{\theta} \mathrm{e}^{2 \sin ^{2} w} \cos ^{3} w \sin w d w\right) \mathrm{e}^{3 \sin ^{2} \theta} \cos \theta, \\
& s_{4,3}(\theta)=-\mathrm{e}^{2 \sin ^{2} \theta}\left(8 \mathrm{e}^{\sin ^{2} \theta} I_{1} \cos ^{2} \theta \sin \theta \int_{0}^{\theta} \mathrm{e}^{2 \sin ^{2} w} d w\right. \\
& -2 I_{2} \int_{0}^{\theta} \mathrm{e}^{3 \sin ^{2} w} \sin w \cos ^{2} w d w+2 I_{1} \int_{0}^{\theta} \mathrm{e}^{3 \sin ^{2} w} \sin w d w \\
& -2 \mathrm{e}^{\sin ^{2} \theta} I_{2} \sin \theta \int_{0}^{\theta} \mathrm{e}^{2 \sin ^{2} w} d w-2 \cos ^{4} \theta I_{3} \int_{0}^{\theta} \mathrm{e}^{3 \sin ^{2} w} \sin w d w \\
& -4 \mathrm{e}^{\sin ^{2} \theta} I_{2} \cos ^{2} \theta \sin \theta \int_{0}^{\theta} \mathrm{e}^{2 \sin ^{2} w} d w+2 \mathrm{e}^{\sin ^{2} \theta} I_{3} \sin \theta \int_{0}^{\theta} \mathrm{e}^{2 \sin ^{2} w} \cos ^{2} w d w \\
& +4 I_{1} \int_{0}^{\theta} \mathrm{e}^{3 \sin ^{2} w} \sin w \cos ^{2} w d w-4 \mathrm{e}^{\sin ^{2} \theta} I_{3} \sin \theta \int_{0}^{\theta} \mathrm{e}^{2 \sin ^{2} w} \cos ^{4} w d w \\
& +2 \cos ^{2} \theta I_{3} \int_{0}^{\theta} \mathrm{e}^{3 \sin ^{2} w} \sin w \cos ^{2} w d w+4 \mathrm{e}^{\sin ^{2} \theta} I_{3} \cos ^{2} \theta \sin \theta \int_{0}^{\theta} \mathrm{e}^{2 \sin ^{2} w} \cos ^{2} w d w
\end{aligned}
$$




$$
\begin{aligned}
& +4 \mathrm{e}^{\sin ^{2} \theta} I_{1} \sin \theta \int_{0}^{\theta} \mathrm{e}^{2 \sin ^{2} w} d w+\cos ^{2} \theta I_{3} \int_{0}^{\theta} \mathrm{e}^{3 \sin ^{2} w} \sin w d w \\
& -I_{2} \int_{0}^{\theta} \mathrm{e}^{3 \sin ^{2} w} \sin w d w-4 \cos ^{4} \theta I_{3} \int_{0}^{\theta} \mathrm{e}^{3 \sin ^{2} w} \sin w \cos ^{2} w d w \\
& \left.-8 \mathrm{e}^{\sin ^{2} \theta} I_{3} \cos ^{2} \theta \sin \theta \int_{0}^{\theta} \mathrm{e}^{2 \sin ^{2} w} \cos ^{4} w d w\right) \frac{1}{2 I_{1}-I_{2}}, \\
& s_{4,4}(\theta)=-\left(8 \mathrm{e}^{\sin ^{2} \theta} \cos ^{2} \theta \int_{0}^{\theta} \mathrm{e}^{2 \sin ^{2} w} \cos ^{3} w \sin w d w\right. \\
& +4 \mathrm{e}^{\sin ^{2} \theta} \cos ^{2} \theta \int_{0}^{\theta} \mathrm{e}^{2 \sin ^{2} w} \sin w \cos w d w+2\left(\int_{0}^{\theta} \mathrm{e}^{3 \sin ^{2} w} \sin w \cos ^{2} w d w\right) \cos \theta \\
& +\left(\int_{0}^{\theta} \mathrm{e}^{3 \sin ^{2} w} \sin w d w\right) \cos \theta+2 \mathrm{e}^{\sin ^{2} \theta} \int_{0}^{\theta} \mathrm{e}^{2 \sin ^{2} w} \sin w \cos w d w \\
& +4\left(\int_{0}^{\theta} \mathrm{e}^{3 \sin ^{2} w} \sin w \cos ^{2} w d w\right) \cos ^{3} \theta+4 \mathrm{e}^{\sin ^{2} \theta} \int_{0}^{\theta} \mathrm{e}^{2 \sin ^{2} w} \cos ^{3} w \sin w d w \\
& \left.+2\left(\int_{0}^{\theta} \mathrm{e}^{3 \sin ^{2} w} \sin w d w\right) \cos ^{3} w\right) \mathrm{e}^{2 \sin ^{2} \theta} \sin \theta \\
& s_{4,5}(\theta)=-\mathrm{e}^{2 \sin ^{2} \theta}\left(-2 I_{1} \int_{0}^{\theta} \mathrm{e}^{3 \sin ^{2} w} \cos w d w+2 \cos ^{4} \theta I_{3} \int_{0}^{\theta} \mathrm{e}^{3 \sin ^{2} w} \cos w d w\right. \\
& -2 I_{2} \int_{0}^{\theta} \mathrm{e}^{3 \sin ^{2} w} \cos ^{3} w d w+4 \mathrm{e}^{\sin ^{2} \theta} I_{3} \cos \theta \int_{0}^{\theta} \mathrm{e}^{2 \sin ^{2} w} \cos ^{4} w d w \\
& -2 \mathrm{e}^{\sin ^{2} \theta} I_{3} \cos \theta \int_{0}^{\theta} \mathrm{e}^{2 \sin ^{2} w} \cos ^{2} w d w-4 \cos ^{4} \theta I_{3} \int_{0}^{\theta} \mathrm{e}^{3 \sin ^{2} w} \cos ^{3} w d w \\
& +2 \mathrm{e}^{\sin ^{2} \theta} I_{2} \cos \theta \int_{0}^{\theta} \mathrm{e}^{2 \sin ^{2} w} d w+I_{2} \int_{0}^{\theta} \mathrm{e}^{3 \sin ^{2} w} \cos w d w \\
& -4 \mathrm{e}^{\sin ^{2} \theta} I_{2} \cos ^{3} w \int_{0}^{\theta} \mathrm{e}^{2 \sin ^{2} w} d w-8 \mathrm{e}^{\sin ^{2} \theta} I_{3} \cos ^{3} w \int_{0}^{\theta} \mathrm{e}^{2 \sin ^{2} w} \cos ^{4} w d w \\
& +4 \mathrm{e}^{\sin ^{2} \theta} I_{3} \cos ^{3} w \int_{0}^{\theta} \mathrm{e}^{2 \sin ^{2} w} \cos ^{2} w d w+4 I_{1} \int_{0}^{\theta} \mathrm{e}^{3 \sin ^{2} w} \cos ^{3} w d w \\
& +8 \mathrm{e}^{\sin ^{2} \theta} I_{1} \cos ^{3} w \int_{0}^{\theta} \mathrm{e}^{2 \sin ^{2} w} d w+2 \cos ^{2} \theta I_{3} \int_{0}^{\theta} \mathrm{e}^{3 \sin ^{2} w} \cos ^{3} w d w \\
& \left.-\cos ^{2} \theta I_{3} \int_{0}^{\theta} \mathrm{e}^{3 \sin ^{2} w} \cos w d w-4 \mathrm{e}^{\sin ^{2} \theta} I_{1} \cos \theta \int_{0}^{\theta} \mathrm{e}^{2 \sin ^{2} w} d w\right) \frac{1}{2 I_{1}-I_{2}}, \\
& s_{4,6}(\theta)=-\left(4 \int_{0}^{\theta} \mathrm{e}^{2 \sin ^{2} w} \cos ^{3} w \sin w d w+4 \cos ^{3} w \mathrm{e}^{-\sin ^{2} \theta} \int_{0}^{\theta} \mathrm{e}^{3 \sin ^{2} w} \sin w \cos ^{2} w d w\right. \\
& +8 \cos ^{2} \theta \int_{0}^{\theta} \mathrm{e}^{2 \sin ^{2} w} \cos ^{3} w \sin w d w-4 \cos ^{2} \theta \int_{0}^{\theta} \mathrm{e}^{2 \sin ^{2} w} \sin w \cos w d w \\
& +2 \cos ^{3} w \mathrm{e}^{-\sin ^{2} \theta} \int_{0}^{\theta} \mathrm{e}^{3 \sin ^{2} w} \sin w d w-\cos \theta \mathrm{e}^{-\sin ^{2} \theta} \int_{0}^{\theta} \mathrm{e}^{3 \sin ^{2} w} \sin w d w \\
& \left.-2 \cos \theta \mathrm{e}^{-\sin ^{2} \theta} \int_{0}^{\theta} \mathrm{e}^{3 \sin ^{2} w} \sin w \cos ^{2} w d w-2 \int_{0}^{\theta} \mathrm{e}^{2 \sin ^{2} w} \sin w \cos w d w\right) \mathrm{e}^{3 \sin ^{2} \theta} \sin \theta .
\end{aligned}
$$


Now we have

$$
\begin{aligned}
& s_{3}(\theta)=s_{3,1}(\theta) a_{00} c_{02}+s_{3,2}(\theta) a_{02} c_{00}+s_{3,3}(\theta) a_{01} c_{01}+s_{3,4}(\theta) a_{00} a_{11}+s_{3,5}(\theta) a_{00} c_{20} \\
& +s_{3,6}(\theta) c_{00} c_{11}+s_{3,7}(\theta) c_{01} c_{10}+s_{3,8}(\theta) a_{00} a_{02}+s_{3,9}(\theta) a_{00} a_{20} \\
& +s_{3,10}(\theta) a_{00} c_{11}+s_{3,11}(\theta) a_{01} c_{10}+s_{3,12}(\theta) a_{11} c_{00}+s_{3,13}(\theta) c_{00} c_{02}+s_{3,14}(\theta) c_{00} c_{20} \\
& +s_{3,15}(\theta) a_{01}^{2}+s_{3,16}(\theta) c_{10}^{2}+s_{3,17}(\theta) c_{01}^{2}+s_{3,18}(\theta) a_{20} c_{00}
\end{aligned}
$$

and $s_{3, i}(\theta)$ for $i=1 \cdots, 18$ satisfying the following expressions:

$$
\begin{aligned}
& s_{3,1}(\theta)=-2 \mathrm{e}^{3 \sin ^{2} \theta} \cos \theta\left[\left(4 \int_{0}^{\theta} \mathrm{e}^{\sin ^{2} w} \sin ^{3} w \cos ^{2} w d w\right) \cos ^{2} \theta\right. \\
& \left.-2 \int_{0}^{\theta} \mathrm{e}^{\sin ^{2} w} \sin ^{3} w \cos ^{2} w d w+2\left(\int_{0}^{\theta} \mathrm{e}^{\sin ^{2} w} \sin ^{3} w d w\right) \cos ^{2} \theta-\int_{0}^{\theta} \mathrm{e}^{\sin ^{2} w} \sin ^{3} w d w\right], \\
& s_{3,2}(\theta)=2 \mathrm{e}^{3 \sin ^{2} \theta} \sin \theta\left[2\left(\int_{0}^{\theta} \mathrm{e}^{\sin ^{2} w} \cos w d w\right) \cos ^{2} \theta\right. \\
& +2 \int_{0}^{\theta} \mathrm{e}^{\sin ^{2} w} \cos ^{5} w d w+4\left(\int_{0}^{\theta} \mathrm{e}^{\sin ^{2} w} \cos ^{5} w d w\right) \cos ^{2} \theta+\int_{0}^{\theta} \mathrm{e}^{\sin ^{2} w^{2}} \cos w d w \\
& \left.-6\left(\int_{0}^{\theta} \mathrm{e}^{\sin ^{2} w} \cos ^{3} w d w\right) \cos ^{2} \theta-3 \int_{0}^{\theta} \mathrm{e}^{\sin ^{2} w} \cos ^{3} w d w\right), \\
& s_{3,3}(\theta)=-\mathrm{e}^{2 \sin ^{2} \theta}\left[-2 I_{1} \cos \theta \sin \theta \int_{0}^{\theta} \mathrm{e}^{2 \sin ^{2} w} d w+4 I_{1} \int_{0}^{\theta} \mathrm{e}^{2 \sin ^{2} w} \cos ^{3} w \sin w d w\right. \\
& -2 I_{1} \int_{0}^{\theta} \mathrm{e}^{2 \sin ^{2} w} \sin w \cos w d w+4 I_{1} \sin \theta\left(\int_{0}^{\theta} \mathrm{e}^{2 \sin ^{2} w} d w\right) \cos ^{3} \theta \\
& +2 I_{3} \cos \theta \sin \theta \int_{0}^{\theta} \mathrm{e}^{2 \sin ^{2} w} \cos ^{4} w d w \\
& +2 I_{3} \sin \theta\left(\int_{0}^{\theta} \mathrm{e}^{2 \sin ^{2} w} \cos ^{2} w d w\right) \cos ^{3} \theta+2 I_{3}\left(\int_{0}^{\theta} \mathrm{e}^{2 \sin ^{2} w} \cos ^{3} w \sin w d w\right) \cos ^{2} \theta \\
& -2 I_{2} \int_{0}^{\theta} \mathrm{e}^{2 \sin ^{2} w} \cos ^{3} w \sin w d w+I_{2} \cos \theta \sin \theta \int_{0}^{\theta} \mathrm{e}^{2 \sin ^{2} w} d w \\
& -4 I_{3} \sin \theta\left(\int_{0}^{\theta} \mathrm{e}^{2 \sin ^{2} w} \cos ^{4} w d w\right) \cos ^{3} \theta-I_{3} \cos \theta \sin \theta \int_{0}^{\theta} \mathrm{e}^{2 \sin ^{2} w} \cos ^{2} w d w \\
& +2 I_{3}\left(\int_{0}^{\theta} \mathrm{e}^{2 \sin ^{2} w} \sin w \cos w d w\right) \cos ^{4} \theta-2 I_{2} \sin \theta\left(\int_{0}^{\theta} \mathrm{e}^{2 \sin ^{2} w} d w\right) \cos ^{3} \theta \\
& -4 I_{3}\left(\int_{0}^{\theta} \mathrm{e}^{2 \sin ^{2} w} \cos ^{3} w \sin w d w\right) \cos ^{3} w \cos ^{4} \theta-I_{3}\left(\int_{0}^{\theta} \mathrm{e}^{2 \sin ^{2} w} \sin w \cos w d w\right) \cos ^{2} \theta \\
& \left.+I_{2} \int_{0}^{\theta} \mathrm{e}^{2 \sin ^{2} w} \sin w \cos w d w\right] \frac{1}{2 I_{1}-I_{2}},
\end{aligned}
$$




$$
\begin{aligned}
& s_{3,4}(\theta)=2 \mathrm{e}^{3 \sin ^{2} \theta} \cos \theta\left[\left(2 \int_{0}^{\theta} \mathrm{e}^{\sin ^{2} w} \cos ^{2} w \sin w d w\right) \cos ^{2} \theta\right. \\
& -\int_{0}^{\theta} \mathrm{e}^{\sin ^{2} w} \cos ^{2} w \sin w d w+2 \int_{0}^{\theta} \mathrm{e}^{\sin ^{2} w} \cos ^{4} w \sin w d w \\
& \left.-4\left(\int_{0}^{\theta} \mathrm{e}^{\sin ^{2} w} \cos ^{4} w \sin w d w\right) \cos ^{2} \theta\right], \\
& s_{3,5}(\theta)=-2 \mathrm{e}^{3 \sin ^{2} \theta} \cos \theta\left[2\left(\int_{0}^{\theta} \mathrm{e}^{\sin ^{2} w} \cos ^{2} w \sin w d w\right) \cos ^{2} \theta\right. \\
& +4\left(\int_{0}^{\theta} \mathrm{e}^{\sin ^{2} w} \cos ^{4} w \sin w d w\right) \cos ^{2} \theta \\
& \left.-\int_{0}^{\theta} \mathrm{e}^{\sin ^{2} w} \cos ^{2} w \sin w d w-2 \int_{0}^{\theta} \mathrm{e}^{\sin ^{2} w} \cos ^{4} w \sin w d w\right], \\
& s_{3,6}(\theta)=-2 \mathrm{e}^{3 \sin ^{2} \theta} \sin \theta\left[\int_{0}^{\theta} \mathrm{e}^{\sin ^{2} w} \cos w d w-2 \int_{0}^{\theta} \mathrm{e}^{\sin ^{2} w} \cos ^{5} w d w\right. \\
& +\int_{0}^{\theta} \mathrm{e}^{\sin ^{2} w} \cos ^{3} w d w+2\left(\int_{0}^{\theta} \mathrm{e}^{\sin ^{2} w} \cos w d w\right) \cos ^{2} \theta \\
& \left.+2\left(\int_{0}^{\theta} \mathrm{e}^{\sin ^{2} w} \cos ^{3} w d w\right) \cos ^{2} \theta-4\left(\int_{0}^{\theta} \mathrm{e}^{\sin ^{2} w} \cos ^{5} w d w\right) \cos ^{2} \theta\right], \\
& s_{3,7}(\theta)=-\mathrm{e}^{2 \sin ^{2} \theta}\left[2 I_{1} \cos \theta \sin \theta \int_{0}^{\theta} \mathrm{e}^{2 \sin ^{2} w} d w+4 I_{1} \sin \theta\left(\int_{0}^{\theta} \mathrm{e}^{2 \sin ^{2} w} d w\right) \cos ^{3} \theta\right. \\
& +2 I_{1} \int_{0}^{\theta} \mathrm{e}^{2 \sin ^{2} w} \sin w \cos w d w+4 I_{1} \int_{0}^{\theta} \mathrm{e}^{2 \sin ^{2} w} \cos ^{3} w \sin w d w \\
& +I_{3} \cos \theta \sin \theta \int_{0}^{\theta} \mathrm{e}^{2 \sin ^{2} w} \cos ^{2} w d w-I_{2} \cos \theta \sin \theta \int_{0}^{\theta} \mathrm{e}^{2 \sin ^{2} w} d w \\
& -2 I_{3} \cos \theta \sin \theta \int_{0}^{\theta} \mathrm{e}^{2 \sin ^{2} w} \cos ^{4} w d w-I_{2} \int_{0}^{\theta} \mathrm{e}^{2 \sin ^{2} w} \sin w \cos w d w \\
& +I_{3}\left(\int_{0}^{\theta} \mathrm{e}^{2 \sin ^{2} w} \sin w \cos w d w\right) \cos ^{2} \theta-2 I_{2} \sin \theta\left(\int_{0}^{\theta} \mathrm{e}^{2 \sin ^{2} w} d w\right) \cos ^{3} \theta \\
& -2 I_{2} \int_{0}^{\theta} \mathrm{e}^{2 \sin ^{2} w} \cos ^{3} w \sin w d w-4 I_{3} \sin \theta\left(\int_{0}^{\theta} \mathrm{e}^{2 \sin ^{2} w} \cos ^{4} w d w\right) \cos ^{3} \theta \\
& +2 I_{3} \sin \theta\left(\int_{0}^{\theta} \mathrm{e}^{2 \sin ^{2} w} \cos ^{2} w d w\right) \cos ^{3} \theta+2 I_{3}\left(\int_{0}^{\theta} \mathrm{e}^{2 \sin ^{2} w} \cos ^{3} w \sin w d w\right) \cos ^{2} \theta \\
& \left.-4 I_{3}\left(\int_{0}^{\theta} \mathrm{e}^{2} \sin ^{2} w \cos ^{3} w \sin w d w\right) \cos ^{4} \theta-2 I_{3}\left(\int_{0}^{\theta} \mathrm{e}^{2 \sin ^{2} w} \sin w \cos w d w\right) \cos ^{4} \theta\right] \frac{1}{2 I_{1}-I_{2}},
\end{aligned}
$$




$$
\begin{aligned}
& s_{3,8}(\theta) \quad=-2 \mathrm{e}^{3 \sin ^{2} \theta} \cos \theta\left[-3 \int_{0}^{\theta} \mathrm{e}^{\sin ^{2} w} \cos ^{3} w d w-2\left(\int_{0}^{\theta} \mathrm{e}^{\sin ^{2} w} \cos w d w\right) \cos ^{2} \theta\right. \\
& +6\left(\int_{0}^{\theta} \mathrm{e}^{\sin ^{2} w} \cos ^{2} w d w\right) \cos ^{2} \theta+2 \int_{0}^{\theta} \mathrm{e}^{\sin ^{2} w^{2}} \cos ^{5} w d w \\
& \left.+\int_{0}^{\theta} \mathrm{e}^{\sin ^{2} w} \cos w d w-4\left(\int_{0}^{\theta} \mathrm{e}^{\sin ^{2} w} \cos ^{5} w d w\right) \cos ^{2} \theta\right] \text {, } \\
& s_{3,9}(\theta) \quad=2 \mathrm{e}^{3 \sin ^{2} \theta} \cos \theta\left[2 \int_{0}^{\theta} \mathrm{e}^{\sin ^{2} w} \cos ^{5} w d w-4\left(\int_{0}^{\theta} \mathrm{e}^{\sin ^{2} w} \cos ^{5} w d w\right) \cos ^{2} \theta\right. \\
& \left.-\int_{0}^{\theta} \mathrm{e}^{\sin ^{2} w} \cos ^{3} w d w+2\left(\int_{0}^{\theta} \mathrm{e}^{\sin ^{2} w} \cos ^{3} w d w\right) \cos ^{2} \theta\right] \text {, } \\
& s_{3,10}(\theta)=-2 \mathrm{e}^{3 \sin ^{2} \theta} \cos \theta\left[2\left(\int_{0}^{\theta} \mathrm{e}^{\sin ^{2} w} \cos ^{3} w d w\right) \cos ^{2} \theta\right. \\
& +2\left(\int_{0}^{\theta} \mathrm{e}^{\sin w^{2}} \cos w d w\right) \cos ^{2} \theta-\int_{0}^{\theta} \mathrm{e}^{\sin ^{2} w} \cos ^{3} w d w \\
& \left.-4\left(\int_{0}^{\theta} \mathrm{e}^{\sin ^{2} w} \cos ^{5} w d w\right) \cos ^{2} \theta-\int_{0}^{\theta} \mathrm{e}^{\sin ^{2} w} \cos w d w+2 \int_{0}^{\theta} \mathrm{e}^{\sin ^{2} w} \cos ^{5} w d w\right], \\
& s_{3,11}(\theta)=2 \mathrm{e}^{2 \sin ^{2} \theta} \sin \theta \cos \theta\left[-4\left(\int_{0}^{\theta} \mathrm{e}^{2 \sin ^{2} w} \cos ^{3} w \sin w d w\right) \cos ^{2} \theta\right. \\
& \left.+\int_{0}^{\theta} \mathrm{e}^{2 \sin ^{2} w} \sin w \cos w d w\right] \\
& s_{3,12}(\theta)=2 \mathrm{e}^{3 \sin ^{2} \theta} \sin \theta\left[2\left(\int_{0}^{\theta} \mathrm{e}^{\sin ^{2} w} \cos ^{2} w \sin w d w\right) \cos ^{2} \theta\right. \\
& +\int_{0}^{\theta} \mathrm{e}^{\sin ^{2} w} \cos ^{2} w \sin w d w-2 \int_{0}^{\theta} \mathrm{e}^{\sin ^{2} w} \cos ^{4} w \sin w d w \\
& \left.-4\left(\int_{0}^{\theta} \mathrm{e}^{\sin ^{2} w} \cos ^{4} w \sin w d w\right) \cos ^{2} \theta\right], \\
& s_{3,13}(\theta)=-2 \mathrm{e}^{3 \sin ^{2} \theta} \sin \theta\left[2 \int_{0}^{\theta} \mathrm{e}^{\sin ^{2} w} \sin ^{3} w \cos ^{2} w d w\right. \\
& +\int_{0}^{\theta} \mathrm{e}^{\sin ^{2} w} \sin ^{3} w d w+4\left(\int_{0}^{\theta} \mathrm{e}^{\sin ^{2} w} \sin ^{3} w \cos ^{2} w d w\right) \cos ^{2} \theta \\
& \left.+2\left(\int_{0}^{\theta} \mathrm{e}^{\sin ^{2} w} \sin ^{3} w d w\right) \cos ^{2} \theta\right]
\end{aligned}
$$




$$
\begin{aligned}
& s_{3,14}(\theta)=-2 \mathrm{e}^{3 \sin ^{2} \theta} \sin \theta\left[2 \int_{0}^{\theta} \mathrm{e}^{\sin ^{2} w} \cos ^{4} w \sin w d w\right. \\
& +4\left(\int_{0}^{\theta} \mathrm{e}^{\sin ^{2} w} \cos ^{4} w \sin w d w\right) \cos ^{2} \theta+\int_{0}^{\theta} \mathrm{e}^{\sin ^{2} w} \cos ^{2} w \sin w d w \\
& \left.+2\left(\int_{0}^{\theta} \mathrm{e}^{\sin ^{2} w} \cos ^{2} w \sin w d w\right) \cos ^{2} \theta\right], \\
& s_{3,15}(\theta)=\mathrm{e}^{2 \sin ^{2} \theta} \sin \theta \cos \theta\left[\left(-4 \int_{0}^{\theta} \mathrm{e}^{2 \sin ^{2} w} \cos ^{3} w \sin w d w\right) \cos ^{2} \theta\right. \\
& -\int_{0}^{\theta} \mathrm{e}^{2 \sin ^{2} w} \sin w \cos w d w+2\left(\int_{0}^{\theta} \mathrm{e}^{2 \sin ^{2} w} \sin w \cos w d w\right) \cos ^{2} \theta \\
& \left.+2 \int_{0}^{\theta} \mathrm{e}^{2 \sin ^{2} w} \cos ^{3} w \sin w d w\right) \\
& s_{3,16}(\theta)=-\mathrm{e}^{2 \sin ^{2} \theta} \sin \theta \cos \theta\left[4\left(\int_{0}^{\theta} \mathrm{e}^{2 \sin ^{2} w} \cos ^{3} w \sin w d w\right) \cos ^{2} \theta\right. \\
& +\int_{0}^{\theta} \mathrm{e}^{2 \sin ^{2} w} \sin w \cos w d w+2\left(\int_{0}^{\theta} \mathrm{e}^{2 \sin ^{2} w} \sin w \cos w d w\right) \cos ^{2} \theta \\
& \left.+2 \int_{0}^{\theta} \mathrm{e}^{2 \sin ^{2} w} \cos ^{3} w \sin w d w\right], \\
& s_{3,17}(\theta)=-\mathrm{e}^{2 \sin ^{2} \theta}\left[-4 I_{1} I_{2} \int_{0}^{\theta} \mathrm{e}^{2 \sin ^{2} w} d w-2 I_{3}^{2}\left(\int_{0}^{\theta} \mathrm{e}^{2 \sin ^{2} w} \cos ^{4} w d w\right) \cos ^{2} \theta\right. \\
& -I_{2} I_{3} \int_{0}^{\theta} \mathrm{e}^{2 \sin ^{2} w} \cos ^{2} w d w+I_{2}{ }^{2} \int_{0}^{\theta} \mathrm{e}^{2 \sin ^{2} w} d w \\
& +2 I_{1} I_{3} \int_{0}^{\theta} \mathrm{e}^{2} \sin ^{2} w \cos ^{2} w d w+2 I_{2} I_{3} \int_{0}^{\theta} \mathrm{e}^{2 \sin ^{2} w} \cos ^{4} w d w \\
& +2 I_{1} I_{3}\left(\int_{0}^{\theta} \mathrm{e}^{2 \sin ^{2} w} d w\right) \cos ^{2} \theta+I_{3}^{2}\left(\int_{0}^{\theta} \mathrm{e}^{2 \sin ^{2} w} \cos ^{2} w d w\right) \cos ^{2} \theta \\
& -I_{2} I_{3}\left(\int_{0}^{\theta} \mathrm{e}^{2 \sin ^{2} w} d w\right) \cos ^{2} \theta+4 I_{1}^{2} \int_{0}^{\theta} \mathrm{e}^{2 \sin ^{2} w} d w-4 I_{1} I_{3} \int_{0}^{\theta} \mathrm{e}^{2 \sin ^{2} w} \cos ^{4} w d w \\
& -2 I_{3}{ }^{2}\left(\int_{0}^{\theta} \mathrm{e}^{2 \sin ^{2} w} \cos ^{2} w d w\right) \cos ^{4} \theta+2 I_{2} I_{3}\left(\int_{0}^{\theta} \mathrm{e}^{2 \sin ^{2} w} d w\right) \cos ^{4} \theta \\
& \left.-4 I_{1} I_{3}\left(\int_{0}^{\theta} \mathrm{e}^{2 \sin ^{2} w} d w\right) \cos ^{4} \theta+4 I_{3}^{2}\left(\int_{0}^{\theta} \mathrm{e}^{2 \sin ^{2} w} \cos ^{4} w d w\right) \cos ^{4} \theta\right] \frac{1}{\left(-I_{2}+2 I_{1}\right)^{2}}, \\
& s_{3,18}(\theta)=2 \mathrm{e}^{3 \sin ^{2} \theta} \sin \theta\left[-2 \int_{0}^{\theta} \mathrm{e}^{\sin ^{2} w} \cos ^{5} w d w+\int_{0}^{\theta} \mathrm{e}^{\sin ^{2} w} \cos ^{3} w d w\right. \\
& \left.+2\left(\int_{0}^{\theta} \mathrm{e}^{\sin ^{2} w} \cos ^{3} w d w\right) \cos ^{2} \theta-4\left(\int_{0}^{\theta} \mathrm{e}^{\sin ^{2} w} \cos ^{5} w d w\right) \cos ^{2} \theta\right] .
\end{aligned}
$$


Now we have

$$
\begin{aligned}
s_{2}(\theta)= & s_{2,1}(\theta) a_{00} c_{30}+s_{2,2}(\theta) a_{20} c_{10}+s_{2,3}(\theta) a_{00} c_{12}+s_{2,4}(\theta) a_{02} c_{10}+s_{2,5}(\theta) a_{12} c_{0,0}+s_{2,6}(\theta) a_{00} a_{21} \\
& +s_{2,7}(\theta) a_{01} a_{20}+s_{2,8}(\theta) a_{01} c_{11}+s_{2,9}(\theta) a_{11} c_{01}+s_{2,10}(\theta) c_{00} c_{21}+s_{2,11}(\theta) c_{01} c_{20}+s_{2,12}(\theta) c_{10} c_{11} \\
& +s_{2,13}(\theta) a_{00} a_{03}+s_{2,14}(\theta) a_{01} a_{02}+s_{2,15}(\theta) c_{00} c_{03}+s_{2,16}(\theta) c_{01} c_{02}+s_{2,17}(\theta) a_{00} a_{12}+s_{2,18}(\theta) a_{03} c_{00} \\
& +s_{2,19}(\theta) a_{11} c_{10}+s_{2,20}(\theta) a_{01} c_{02}+s_{2,21}(\theta) a_{01} c_{20}+s_{2,22}(\theta) a_{01} a_{11}+s_{2,23}(\theta) a_{00} c_{03} \\
& +s_{2,24}(\theta) a_{00} c_{21}+s_{2,25}(\theta) a_{02} c_{01}+s_{2,26}(\theta) a_{20} c_{01}+s_{2,27}(\theta) a_{21} c_{00} \\
& +s_{2,28}(\theta) c_{00} c_{12}+s_{2,29}(\theta) c_{00} c_{30}+s_{2,30}(\theta) c_{01} c_{11}+s_{2,31}(\theta) c_{02} c_{10}+s_{2,32}(\theta) c_{10} c_{20}
\end{aligned}
$$

and $s_{2, i}(\theta)$ for $i=1, \cdots, 32$ satisfying the following expressions:

$$
\begin{aligned}
& s_{2,1}(\theta)=\frac{1}{6}\left(12 \cos ^{2} \theta \sin \theta \mathrm{e}^{-3 \sin ^{2} \theta} \int_{0}^{\theta} \mathrm{e}^{3 \sin ^{2} w} \cos ^{3} w d w-12 \cos ^{4} \theta \sin \theta\left(\int_{0}^{\theta} \mathrm{e}^{3 \sin ^{2} w} \cos w d w\right) \mathrm{e}^{-3 \sin ^{2} \theta}\right. \\
& +2 \cos ^{6} \theta-14 \cos ^{2} \theta-6 \cos ^{2} \theta \sin \theta\left(\int_{0}^{\theta} \mathrm{e}^{3 \sin ^{2} w} \cos w d w\right) \mathrm{e}^{-3 \sin ^{2} \theta} \\
& \left.+24 \cos ^{4} \theta \sin \theta \mathrm{e}^{-3 \sin ^{2} \theta} \int_{0}^{\theta} \mathrm{e}^{3 \sin ^{2} w} \cos ^{3} w d w-3 \cos ^{4} \theta+7+8 \cos ^{8} \theta\right) \mathrm{e}^{3 \sin ^{2} \theta} \cos \theta, \\
& s_{2,2}(\theta)=\left(-2 \int_{0}^{\theta} \mathrm{e}^{\sin ^{2} w} \cos ^{5} w d w+\int_{0}^{\theta} \mathrm{e}^{\sin ^{2} w} \cos ^{3} w d w\right. \\
& \left.+2\left(\int_{0}^{\theta} \mathrm{e}^{\sin ^{2} w} \cos ^{3} w d w\right) \cos ^{2} \theta-4\left(\int_{0}^{\theta} \mathrm{e}^{\sin ^{2} w} \cos ^{5} w d w\right) \cos ^{2} \theta\right) \mathrm{e}^{2 \sin ^{2} \theta} \sin \theta \cos \theta, \\
& s_{2,3}(\theta)=-\frac{1}{6}\left(-12 \sin \theta \mathrm{e}^{-3 \sin ^{2} \theta} \int_{0}^{\theta} \mathrm{e}^{3 \sin ^{2} w} \cos ^{3} w d w-9 \cos ^{4} \theta\right. \\
& -12 \cos ^{4} \theta \sin \theta\left(\int_{0}^{\theta} \mathrm{e}^{3 \sin ^{2} w} \cos w d w\right) \mathrm{e}^{-3 \sin ^{2} \theta}+6 \cos ^{2} \theta \sin \theta\left(\int_{0}^{\theta} \mathrm{e}^{3} \sin ^{2} w \cos w d w\right) \mathrm{e}^{-3 \sin ^{2} \theta} \\
& -10 \cos ^{6} \theta-12 \cos ^{2} \theta \sin \theta \mathrm{e}^{-3 \sin ^{2} \theta} \int_{0}^{\theta} \mathrm{e}^{3 \sin ^{2} w} \cos ^{3} w d w+6 \sin \theta\left(\int_{0}^{\theta} \mathrm{e}^{3 \sin ^{2} w} \cos w d w\right) \mathrm{e}^{-3 \sin ^{2} \theta} \\
& \left.+8 \cos ^{8} \theta+16 \cos ^{2} \theta+24 \cos ^{4} \theta \sin \theta \mathrm{e}^{-3 \sin ^{2} \theta} \int_{0}^{\theta} \mathrm{e}^{3 \sin ^{2} w} \cos ^{3} w d w-5\right) \mathrm{e}^{3 \sin ^{2} \theta} \cos \theta, \\
& s_{2,4}(\theta)=-\left(-4\left(\int_{0}^{\theta} \mathrm{e}^{\sin ^{2} w} \cos ^{5} w d w\right) \cos ^{2} \theta+3 \int_{0}^{\theta} \mathrm{e}^{\sin ^{2} w} \cos ^{3} w d w+6\left(\int_{0}^{\theta} \mathrm{e}^{\sin ^{2} w} \cos ^{3} w d w\right) \cos ^{2} \theta\right. \\
& \left.-\int_{0}^{\theta} \mathrm{e}^{\sin ^{2} w} \cos w d w-2\left(\int_{0}^{\theta} \mathrm{e}^{\sin ^{2} w} \cos w d w\right) \cos ^{2} \theta-2 \int_{0}^{\theta} \mathrm{e}^{\sin ^{2} w} \cos ^{5} w d w\right) \mathrm{e}^{2 \sin ^{2} \theta} \sin \theta \cos \theta, \\
& s_{2,5}(\theta)=-\frac{1}{3}\left(6 \cos ^{4} \theta \int_{0}^{\theta} \mathrm{e}^{3 \sin ^{2} w} \sin w d w+4 \mathrm{e}^{3 \sin ^{2} \theta} \cos ^{7} \theta-18\left(\int_{0}^{\theta} \mathrm{e}^{3 \sin ^{2} w} \sin w \cos ^{2} w d w\right) \cos ^{2} \theta\right. \\
& -6 \mathrm{e}^{3 \sin ^{2} \theta} \cos ^{5} \theta-9\left(\int_{0}^{\theta} \mathrm{e}^{3 \sin ^{2} w} \sin w d w\right) \cos ^{2} \theta+2 \mathrm{e}^{3 \sin ^{2} \theta} \cos \theta+3 \int_{0}^{\theta} \mathrm{e}^{3 \sin ^{2} w} \sin w d w \\
& \left.+12 \cos ^{4} \theta \int_{0}^{\theta} \mathrm{e}^{3 \sin ^{2} w} \sin w \cos ^{2} w d w+6 \int_{0}^{\theta} \mathrm{e}^{3 \sin ^{2} w} \sin w \cos ^{2} w d w\right) \cos ^{2} \theta,
\end{aligned}
$$




$$
\begin{aligned}
& s_{2,6}(\theta)=\frac{1}{6}\left(-12 \cos ^{2} \theta \sin \theta \mathrm{e}^{-3 \sin ^{2} \theta} \int_{0}^{\theta} \mathrm{e}^{3 \sin ^{2} w} \cos ^{3} w d w+1-2 \cos ^{2} \theta\right. \\
& -10 \cos ^{6} \theta-12 \cos ^{4} \theta \sin \theta\left(\int_{0}^{\theta} \mathrm{e}^{3 \sin ^{2} w} \cos w d w\right) \mathrm{e}^{-3 \sin ^{2} \theta}+3 \cos ^{4} \theta \\
& +24 \cos ^{4} \theta \sin \theta \mathrm{e}^{-3 \sin ^{2} \theta} \int_{0}^{\theta} \mathrm{e}^{3 \sin ^{2} w} \cos ^{3} w d w \\
& \left.+6 \cos ^{2} \theta \sin \theta\left(\int_{0}^{\theta} \mathrm{e}^{3 \sin ^{2} w} \cos w d w\right) \mathrm{e}^{-3 \sin ^{2} \theta}+8 \cos ^{8} \theta\right) \mathrm{e}^{3 \sin ^{2} \theta} \cos \theta, \\
& s_{2,7}(\theta)=\left(2 \int_{0}^{\theta} \mathrm{e}^{\sin ^{2} w} \cos ^{5} w d w-4\left(\int_{0}^{\theta} \mathrm{e}^{\sin ^{2} w} \cos ^{5} w d w\right) \cos ^{2} \theta\right. \\
& \left.-\int_{0}^{\theta} \mathrm{e}^{\sin ^{2} w} \cos ^{3} w d w+2\left(\int_{0}^{\theta} \mathrm{e}^{\sin ^{2} w} \cos ^{3} w d w\right) \cos ^{2} \theta\right) \mathrm{e}^{2 \sin ^{2} \theta} \sin \theta \cos \theta, \\
& s_{2,8}(\theta)=-\left(2\left(\int_{0}^{\theta} \mathrm{e}^{\sin ^{2} w} \cos ^{3} w d w\right) \cos ^{2} \theta+2\left(\int_{0}^{\theta} \mathrm{e}^{\sin ^{2} w} \cos w d w\right) \cos ^{2} \theta-\int_{0}^{\theta} \mathrm{e}^{\sin ^{2} w} \cos ^{3} w d w\right. \\
& \left.-4\left(\int_{0}^{\theta} \mathrm{e}^{\sin ^{2} w} \cos ^{5} w d w\right) \cos ^{2} \theta-\int_{0}^{\theta} \mathrm{e}^{\sin ^{2} w} \cos w d w+2 \int_{0}^{\theta} \mathrm{e}^{\sin ^{2} w} \cos ^{5} w d w\right) \mathrm{e}^{2 \sin ^{2} \theta} \sin \theta \cos \theta, \\
& s_{2,9}(\theta)=\mathrm{e}^{2 \sin ^{2} \theta}\left(-I_{2} \int_{0}^{\theta} \mathrm{e}^{\sin ^{2} w} \cos ^{2} w \sin w d w+2 I_{1} \int_{0}^{\theta} \mathrm{e}^{\sin ^{2} w} \cos ^{2} w \sin w d w\right. \\
& -4 I_{1} \int_{0}^{\theta} \mathrm{e}^{\sin ^{2} w} \cos ^{4} w \sin w d w-2 I_{3} \cos ^{2} \theta \int_{0}^{\theta} \mathrm{e}^{\sin ^{2} w} \cos ^{4} w \sin w d w \\
& +I_{3} \cos ^{2} \theta \int_{0}^{\theta} \mathrm{e}^{\sin ^{2} w} \cos ^{2} w \sin w d w-2 I_{3} \cos ^{4} \theta \int_{0}^{\theta} \mathrm{e}^{\sin ^{2} w} \cos ^{2} w \sin w d w \\
& \left.+4 I_{3} \cos ^{4} \theta \int_{0}^{\theta} \mathrm{e}^{\sin ^{2} w} \cos ^{4} w \sin w d w+2 I_{2} \int_{0}^{\theta} \mathrm{e}^{\sin ^{2} w} \cos ^{4} w \sin w d w\right) \frac{1}{2 I_{1}-I_{2}}, \\
& s_{2,10}(\theta)=-\frac{1}{3} \cos ^{2} \theta\left(-6\left(\int_{0}^{\theta} \mathrm{e}^{3 \sin ^{2} w} \sin w d w\right) \cos ^{2} \theta-2 \mathrm{e}^{3 \sin ^{2} \theta} \cos \theta-3 \int_{0}^{\theta} \mathrm{e}^{3 \sin ^{2} w} \sin w d w\right. \\
& -6 \int_{0}^{\theta} \mathrm{e}^{3 \sin ^{2} w} \sin w \cos ^{2} w d w-12\left(\int_{0}^{\theta} \mathrm{e}^{3 \sin ^{2} w} \sin w \cos ^{2} w d w\right) \cos ^{2} \theta-6 \mathrm{e}^{3 \sin ^{2} \theta} \cos ^{3} \theta \\
& \left.+8 \mathrm{e}^{3 \sin ^{2} \theta} \cos ^{7} \theta+12 \cos ^{4} \theta \int_{0}^{\theta} \mathrm{e}^{3 \sin ^{2} w} \sin w d w+24 \cos ^{4} \theta \int_{0}^{\theta} \mathrm{e}^{3 \sin ^{2} w} \sin w \cos ^{2} w d w\right), \\
& s_{2,11}(\theta)=-\mathrm{e}^{2 \sin ^{2} \theta}\left(2 I_{1} \int_{0}^{\theta} \mathrm{e}^{\sin ^{2} w} \cos ^{2} w \sin w d w+2 I_{3} \cos ^{2} \theta \int_{0}^{\theta} \mathrm{e}^{\sin ^{2} w} \cos ^{4} w \sin w d w\right. \\
& -I_{2} \int_{0}^{\theta} \mathrm{e}^{\sin ^{2} w} \cos ^{2} w \sin w d w+I_{3} \cos ^{2} \theta \int_{0}^{\theta} \mathrm{e}^{\sin ^{2} w} \cos ^{2} w \sin w d w \\
& -2 I_{3} \cos ^{4} \theta \int_{0}^{\theta} \mathrm{e}^{\sin ^{2} w} \cos ^{2} w \sin w d w-4 I_{3} \cos ^{4} \theta \int_{0}^{\theta} \mathrm{e}^{\sin ^{2} w} \cos ^{4} w \sin w d w \\
& \left.-2 I_{2} \int_{0}^{\theta} \mathrm{e}^{\sin ^{2} w} \cos ^{4} w \sin w d w+4 I_{1} \int_{0}^{\theta} \mathrm{e}^{\sin ^{2} w} \cos ^{4} w \sin w d w\right) \frac{1}{2 I_{1}-I_{2}},
\end{aligned}
$$




$$
\begin{aligned}
& s_{2,12}(\theta)=-\left(\int_{0}^{\theta} \mathrm{e}^{\sin ^{2} w} \cos w d w-2 \int_{0}^{\theta} \mathrm{e}^{\sin ^{2} w} \cos ^{5} w d w+\int_{0}^{\theta} \mathrm{e}^{\sin ^{2} w} \cos ^{3} w d w\right. \\
& +2\left(\int_{0}^{\theta} \mathrm{e}^{\sin ^{2} w} \cos w d w\right) \cos ^{2} \theta+2\left(\int_{0}^{\theta} \mathrm{e}^{\sin ^{2} w} \cos ^{3} w d w\right) \cos ^{2} \theta \\
& \left.-4\left(\int_{0}^{\theta} \mathrm{e}^{\sin ^{2} w} \cos ^{5} w d w\right) \cos ^{2} \theta\right) \mathrm{e}^{2 \sin ^{2} \theta} \cos \theta \sin \theta, \\
& s_{2,13}(\theta)=-\frac{1}{6}\left(-12 \cos ^{4} \theta \sin \theta \int_{0}^{\theta} \mathrm{e}^{3 \sin ^{2} w} \cos w d w-6 \sin \theta \int_{0}^{\theta} \mathrm{e}^{3 \sin ^{2} w} \cos w d w\right. \\
& +24 \cos ^{4} \theta \sin \theta \int_{0}^{\theta} \mathrm{e}^{3 \sin ^{2} w} \cos ^{3} w d w+12 \sin \theta \int_{0}^{\theta} \mathrm{e}^{3 \sin ^{2} w} \cos ^{3} w d w \\
& -36 \cos ^{2} \theta \sin \theta \int_{0}^{\theta} \mathrm{e}^{3 \sin ^{2} w} \cos ^{3} w d w-22 \mathrm{e}^{3 \sin ^{2} \theta} \cos ^{6} \theta+\mathrm{e}^{3 \sin ^{2} \theta} \\
& -8 \mathrm{e}^{3 \sin ^{2} \theta} \cos ^{2} \theta+8 \mathrm{e}^{3 \sin ^{2} \theta} \cos ^{8} \theta+18 \sin \theta \cos ^{2} \theta \int_{0}^{\theta} \mathrm{e}^{3 \sin ^{2} w} \cos w d w \\
& \left.+21 \mathrm{e}^{3 \sin ^{2} \theta} \cos ^{4} \theta\right) \cos \theta \\
& s_{2,14}(\theta)=-\left(-3 \int_{0}^{\theta} \mathrm{e}^{\sin ^{2} w} \cos ^{3} w d w-2\left(\int_{0}^{\theta} \mathrm{e}^{\sin ^{2} w} \cos w d w\right) \cos ^{2} \theta+6\left(\int_{0}^{\theta} \mathrm{e}^{\sin ^{2} w} \cos ^{3} w d w\right) \cos ^{2} \theta\right. \\
& \left.+2 \int_{0}^{\theta} \mathrm{e}^{\sin ^{2} w} \cos ^{5} w d w+\int_{0}^{\theta} \mathrm{e}^{\sin ^{2} w} \cos w d w-4\left(\int_{0}^{\theta} \mathrm{e}^{\sin ^{2} w} \cos ^{5} w d w\right) \cos ^{2} \theta\right) \mathrm{e}^{2 \sin ^{2} \theta} \cos \theta \sin \theta \\
& s_{2,15}(\theta)=-2 \mathrm{e}^{3 \sin ^{2} \theta} \cos ^{7} \theta-\cos ^{4} \theta \int_{0}^{\theta} \mathrm{e}^{3 \sin ^{2} w} \sin w d w-2 \cos ^{6} \theta \int_{0}^{\theta} \mathrm{e}^{3 \sin ^{2} w} \sin w d w \\
& -2 \mathrm{e}^{3 \sin ^{2} \theta} \cos ^{5} \theta-2 \cos ^{4} \theta \int_{0}^{\theta} \mathrm{e}^{3 \sin ^{2} w} \sin w \cos ^{2} w d w \\
& +\frac{10}{3} \mathrm{e}^{3 \sin ^{2} \theta} \cos ^{3} \theta-4 \cos ^{6} \theta \int_{0}^{\theta} \mathrm{e}^{3 \sin ^{2} w} \sin w \cos ^{2} w d w-\frac{4}{3} \cos ^{9} \theta \mathrm{e}^{3 \sin ^{2} \theta} \\
& +2 \mathrm{e}^{3 \sin ^{2} \theta} \cos \theta+\int_{0}^{\theta} \mathrm{e}^{3 \sin ^{2} w} \sin w d w+2 \int_{0}^{\theta} \mathrm{e}^{3 \sin ^{2} w} \sin w \cos ^{2} w d w, \\
& s_{2,16}(\theta)=-\mathrm{e}^{2 \sin ^{2} \theta}\left(-2 I_{2} \int_{0}^{\theta} \mathrm{e}^{\sin ^{2} w} \sin ^{3} w \cos ^{2} w d w+2 I_{3} \cos ^{2} \theta \int_{0}^{\theta} \mathrm{e}^{\sin ^{2} w} \sin ^{3} w \cos ^{2} w d w\right. \\
& +2 I_{1} \int_{0}^{\theta} \mathrm{e}^{\sin ^{2} w} \sin ^{3} w d w+4 I_{1} \int_{0}^{\theta} \mathrm{e}^{\sin ^{2} w} \sin ^{3} w \cos ^{2} w d w-4 I_{3} \cos ^{4} \theta \int_{0}^{\theta} \mathrm{e}^{\sin ^{2} w} \sin ^{3} w \cos ^{2} w d w \\
& \left.+I_{3} \cos ^{2} \theta \int_{0}^{\theta} \mathrm{e}^{\sin ^{2} w} \sin ^{3} w d w-I_{2} \int_{0}^{\theta} \mathrm{e}^{\sin ^{2} w} \sin ^{3} w d w-2 I_{3} \cos ^{4} \theta \int_{0}^{\theta} \mathrm{e}^{\sin ^{2} w} \sin ^{3} w d w\right) \frac{1}{2 I_{1}-I_{2}}, \\
& s_{2,17}(\theta)=\frac{1}{3}\left(-6 \int_{0}^{\theta} \mathrm{e}^{3 \sin ^{2} w} \cos ^{3} w d w+3 \int_{0}^{\theta} \mathrm{e}^{3 \sin ^{2} w} \cos w d w+2 \mathrm{e}^{3 \sin ^{2} \theta} \cos ^{2} \theta \sin \theta\right. \\
& +6 \cos ^{4} \theta \int_{0}^{\theta} \mathrm{e}^{3 \sin ^{2} w} \cos w d w+4 \mathrm{e}^{3 \sin ^{2} \theta} \cos ^{6} \theta \sin \theta-6 \mathrm{e}^{3 \sin ^{2} \theta} \cos ^{4} \theta \sin \theta \\
& \left.-9 \cos ^{2} \theta \int_{0}^{\theta} \mathrm{e}^{3 \sin ^{2} w} \cos w d w+\left(18 \cos ^{2} \theta-12 \cos ^{4} \theta\right) \int_{0}^{\theta} \mathrm{e}^{3 \sin ^{2} w} \cos ^{3} w d w\right) \cos ^{2} \theta
\end{aligned}
$$




$$
\begin{aligned}
& s_{2,18}(\theta)=-\frac{1}{6}\left(8 \mathrm{e}^{3 \sin ^{2} \theta} \cos ^{8} \theta-14 \mathrm{e}^{3 \sin ^{2} \theta} \cos ^{6} \theta-\mathrm{e}^{3 \sin ^{2} \theta}+12 \cos ^{5} \theta \int_{0}^{\theta} \mathrm{e}^{3 \sin ^{2} w} \sin w d w\right. \\
& +24 \cos ^{5} \theta \int_{0}^{\theta} \mathrm{e}^{3 \sin ^{2} w} \sin w \cos ^{2} w d w+4 \mathrm{e}^{3 \sin ^{2} \theta} \cos ^{2} \theta-36 \cos ^{3} \theta \int_{0}^{\theta} \mathrm{e}^{3 \sin ^{2} w} \sin w \cos ^{2} w d w \\
& -18 \cos ^{3} \theta \int_{0}^{\theta} \mathrm{e}^{3 \sin ^{2} w} \sin w d w+6 \cos \theta \int_{0}^{\theta} \mathrm{e}^{3 \sin ^{2} w} \sin w d w \\
& \left.+12 \cos \theta \int_{0}^{\theta} \mathrm{e}^{3 \sin ^{2} w} \sin w \cos ^{2} w d w+3 \mathrm{e}^{3 \sin ^{2} \theta} \cos ^{4} \theta\right) \sin \theta, \\
& s_{2,19}(\theta)=\left(2\left(\int_{0}^{\theta} \mathrm{e}^{\sin ^{2} w} \cos ^{2} w \sin w d w\right) \cos ^{2} \theta+\int_{0}^{\theta} \mathrm{e}^{\sin ^{2} w} \cos ^{2} w \sin w d w\right. \\
& \left.-2 \int_{0}^{\theta} \mathrm{e}^{\sin ^{2} w} \cos ^{4} w \sin w d w-4\left(\int_{0}^{\theta} \mathrm{e}^{\sin ^{2} w} \cos ^{4} w \sin w d w\right) \cos ^{2} \theta\right) \mathrm{e}^{2 \sin ^{2} \theta} \cos \theta \sin \theta, \\
& s_{2,20}(\theta)=-\left(4\left(\int_{0}^{\theta} \mathrm{e}^{\sin ^{2} w} \sin ^{3} w \cos ^{2} w d w\right) \cos ^{2} \theta-2 \int_{0}^{\theta} \mathrm{e}^{\sin ^{2} w} \sin ^{3} w \cos ^{2} w d w\right. \\
& \left.-\int_{0}^{\theta} \mathrm{e}^{\sin ^{2} w} \sin ^{3} w d w+2\left(\int_{0}^{\theta} \mathrm{e}^{\sin ^{2} w} \sin ^{3} w d w\right) \cos ^{2} \theta\right) \mathrm{e}^{2 \sin ^{2} \theta} \cos \theta \sin \theta \\
& s_{2,21}(\theta)=-\left(2\left(\int_{0}^{\theta} \mathrm{e}^{\sin ^{2} w} \cos ^{2} w \sin w d w\right) \cos ^{2} \theta+4\left(\int_{0}^{\theta} \mathrm{e}^{\sin ^{2} w} \cos ^{4} w \sin w d w\right) \cos ^{2} \theta\right. \\
& \left.-\int_{0}^{\theta} \mathrm{e}^{\sin ^{2} w} \cos ^{2} w \sin w d w-2 \int_{0}^{\theta} \mathrm{e}^{\sin ^{2} w} \cos ^{4} w \sin w d w\right) \mathrm{e}^{2 \sin ^{2} \theta} \cos \theta \sin \theta \\
& s_{2,22}(\theta)=\left(2\left(\int_{0}^{\theta} \mathrm{e}^{\sin ^{2} w} \cos ^{2} w \sin w d w\right) \cos ^{2} \theta-\int_{0}^{\theta} \mathrm{e}^{\sin ^{2} w} \cos ^{2} w \sin w d w\right. \\
& \left.+2 \int_{0}^{\theta} \mathrm{e}^{\sin ^{2} w} \cos ^{4} w \sin w d w-4\left(\int_{0}^{\theta} \mathrm{e}^{\sin ^{2} w} \cos ^{4} w \sin w d w\right) \cos ^{2} \theta\right) \mathrm{e}^{2 \sin ^{2} \theta} \cos \theta \sin \theta, \\
& s_{2,23}(\theta)=2 \mathrm{e}^{3 \sin ^{2} \theta} \cos ^{6} \theta \sin \theta+\frac{4}{3} \mathrm{e}^{3 \sin ^{2} \theta} \cos ^{8} \theta \sin \theta+2 \int_{0}^{\theta} \mathrm{e}^{3 \sin ^{2} w} \cos ^{3} w d w+\frac{8}{3} \mathrm{e}^{3 \sin ^{2} \theta} \cos ^{4} \theta \sin \theta \\
& -2 \cos ^{4} \theta \int_{0}^{\theta} \mathrm{e}^{3} \sin ^{2} w \cos ^{3} w d w-2 \mathrm{e}^{3 \sin ^{2} \theta} \cos ^{2} \theta \sin \theta+2 \cos ^{6} \theta \int_{0}^{\theta} \mathrm{e}^{3 \sin ^{2} w} \cos w d w \\
& -4 \cos ^{6} \theta \int_{0}^{\theta} \mathrm{e}^{3 \sin ^{2} w} \cos ^{3} w d w-\int_{0}^{\theta} \mathrm{e}^{3 \sin ^{2} w} \cos w d w+\cos ^{4} \theta \int_{0}^{\theta} \mathrm{e}^{3} \sin ^{2} w \cos w d w, \\
& s_{2,24}(\theta)=\frac{1}{3}\left(6 \int_{0}^{\theta} \mathrm{e}^{3 \sin ^{2} w} \cos ^{3} w d w+12 \cos ^{4} \theta \int_{0}^{\theta} \mathrm{e}^{3 \sin ^{2} w} \cos w d w+12 \cos ^{2} \theta \int_{0}^{\theta} \mathrm{e}^{3 \sin ^{2} w} \cos ^{3} w d w\right. \\
& -3 \int_{0}^{\theta} \mathrm{e}^{3 \sin ^{2} w} \cos w d w+8 \mathrm{e}^{3 \sin ^{2} \theta} \cos ^{6} \theta \sin \theta-24 \cos ^{4} \theta \int_{0}^{\theta} \mathrm{e}^{3 \sin ^{2} w} \cos ^{3} w d w \\
& \left.-6 \cos ^{2} \theta \int_{0}^{\theta} \mathrm{e}^{3 \sin ^{2} w} \cos w d w-2 \mathrm{e}^{3 \sin ^{2} \theta} \cos ^{2} \theta \sin \theta\right) \cos ^{2} \theta,
\end{aligned}
$$




$$
\begin{aligned}
& s_{2,25}(\theta)=-\mathrm{e}^{2} \sin ^{2} \theta\left(3 I_{3} \cos ^{2} \theta \int_{0}^{\theta} \mathrm{e}^{\sin ^{2} w} \cos ^{3} w d w+2 I_{3} \cos ^{4} \theta \int_{0}^{\theta} \mathrm{e}^{\sin ^{2} w} \cos w d w\right. \\
& +6 I_{1} \int_{0}^{\theta} \mathrm{e}^{\sin ^{2} w} \cos ^{3} w d w-4 I_{1} \int_{0}^{\theta} \mathrm{e}^{\sin ^{2} w} \cos ^{5} w d w+4 I_{3} \cos ^{4} \theta \int_{0}^{\theta} \mathrm{e}^{\sin ^{2} w} \cos ^{5} w d w \\
& +2 I_{2} \int_{0}^{\theta} \mathrm{e}^{\sin ^{2} w} \cos ^{5} w d w+I_{2} \int_{0}^{\theta} \mathrm{e}^{\sin ^{2} w} \cos w d w-6 I_{3} \cos ^{4} \theta \int_{0}^{\theta} \mathrm{e}^{\sin ^{2} w} \cos ^{3} w d w \\
& -2 I_{3} \cos ^{2} \theta \int_{0}^{\theta} \mathrm{e}^{\sin ^{2} w} \cos ^{5} w d w-2 I_{1} \int_{0}^{\theta} \mathrm{e}^{\sin ^{2} w} \cos w d w \\
& \left.-3 I_{2} \int_{0}^{\theta} \mathrm{e}^{\sin ^{2} w} \cos ^{3} w d w-I_{3} \cos ^{2} \theta \int_{0}^{\theta} \mathrm{e}^{\sin ^{2} w} \cos w d w\right) \frac{1}{2 I_{1}-I_{2}}, \\
& s_{2,26}(\theta)=\mathrm{e}^{2 \sin ^{2} \theta}\left(-2 I_{3} \cos ^{4} \theta \int_{0}^{\theta} \mathrm{e}^{\sin ^{2} w} \cos ^{3} w d w+4 I_{3} \cos ^{4} \theta \int_{0}^{\theta} \mathrm{e}^{\sin ^{2} w} \cos ^{5} w d w\right. \\
& -I_{2} \int_{0}^{\theta} \mathrm{e}^{\sin ^{2} w} \cos ^{3} w d w-2 I_{3} \cos ^{2} \theta \int_{0}^{\theta} \mathrm{e}^{\sin ^{2} w} \cos ^{5} w d w+2 I_{2} \int_{0}^{\theta} \mathrm{e}^{\sin ^{2} w} \cos ^{5} w d w \\
& \left.+2 I_{1} \int_{0}^{\theta} \mathrm{e}^{\sin ^{2} w} \cos ^{3} w d w-4 I_{1} \int_{0}^{\theta} \mathrm{e}^{\sin ^{2} w} \cos ^{5} w d w+I_{3} \cos ^{2} \theta \int_{0}^{\theta} \mathrm{e}^{\sin ^{2} w} \cos ^{3} w d w\right) \frac{1}{2 I_{1}-I_{2}}, \\
& s_{2,27}(\theta)=\frac{1}{6}\left(-6 \cos ^{3} \theta \int_{0}^{\theta} \mathrm{e}^{3 \sin ^{2} w} \sin w d w+12 \cos ^{5} \theta \int_{0}^{\theta} \mathrm{e}^{3 \sin ^{2} w} \sin w d w-3 \mathrm{e}^{3 \sin ^{2} \theta} \cos ^{4} \theta\right. \\
& -2 \mathrm{e}^{3 \sin ^{2} \theta} \cos ^{2} \theta-2 \mathrm{e}^{3 \sin ^{2} \theta} \cos ^{6} \theta-\mathrm{e}^{3 \sin ^{2} \theta}+24 \cos ^{5} \theta \int_{0}^{\theta} \mathrm{e}^{3 \sin ^{2} w} \sin w \cos ^{2} w d w \\
& \left.-12 \cos ^{3} \theta \int_{0}^{\theta} \mathrm{e}^{3 \sin ^{2} w} \sin w \cos ^{2} w d w+8 \mathrm{e}^{3 \sin ^{2} \theta} \cos ^{8} \theta\right) \sin \theta, \\
& s_{2,28}(\theta)=-\frac{1}{6}\left(5 \mathrm{e}^{3 \sin ^{2} \theta}+24 \cos ^{5} \theta \int_{0}^{\theta} \mathrm{e}^{3 \sin ^{2} w} \sin w \cos ^{2} w d w\right. \\
& -12 \cos ^{3} \theta \int_{0}^{\theta} \mathrm{e}^{3 \sin ^{2} w} \sin w \cos ^{2} w d w-12 \cos \theta \int_{0}^{\theta} \mathrm{e}^{3 \sin ^{2} w} \sin w \cos ^{2} w d w \\
& -6 \cos ^{3} \theta \int_{0}^{\theta} \mathrm{e}^{3 \sin ^{2} w} \sin w d w-15 \mathrm{e}^{3 \sin ^{2} \theta} \cos ^{4} \theta-6 \cos \theta \int_{0}^{\theta} \mathrm{e}^{3 \sin ^{2} w} \sin w d w \\
& +4 \mathrm{e}^{3 \sin ^{2} \theta} \cos ^{2} \theta+8 \mathrm{e}^{3 \sin ^{2} \theta} \cos ^{8} \theta+12 \cos ^{5} \theta \int_{0}^{\theta} \mathrm{e}^{3 \sin ^{2} w} \sin w d w \\
& \left.-2 \mathrm{e}^{3 \sin ^{2} \theta} \cos ^{6} \theta\right) \sin \theta, \\
& s_{2,29}(\theta)=\frac{1}{6}\left(-14 \mathrm{e}^{3 \sin ^{2} \theta} \cos ^{2} \theta+10 \mathrm{e}^{3 \sin ^{2} \theta} \cos ^{6} \theta+12 \cos ^{5} \theta \int_{0}^{\theta} \mathrm{e}^{3 \sin ^{2} w} \sin w d w\right. \\
& +6 \cos ^{3} \theta \int_{0}^{\theta} \mathrm{e}^{3 \sin ^{2} w} \sin w d w+12 \cos ^{3} \theta \int_{0}^{\theta} \mathrm{e}^{3 \sin ^{2} w} \sin w \cos ^{2} w d w+8 \mathrm{e}^{3 \sin ^{2} \theta} \cos ^{8} \theta \\
& \left.+3 \mathrm{e}^{3 \sin ^{2} \theta} \cos ^{4} \theta-7 \mathrm{e}^{3 \sin ^{2} \theta}+24 \cos ^{5} \theta \int_{0}^{\theta} \mathrm{e}^{3 \sin ^{2} w} \sin w \cos ^{2} w d w\right) \sin \theta,
\end{aligned}
$$




$$
\begin{aligned}
s_{2,30}(\theta)= & -\mathrm{e}^{2} \sin ^{2} \theta\left(-4 I_{1} \int_{0}^{\theta} \mathrm{e}^{\sin ^{2} w} \cos ^{5} w d w-2 I_{3} \cos ^{4} \theta \int_{0}^{\theta} \mathrm{e}^{\sin ^{2} w} \cos w d w\right. \\
& -2 I_{3} \cos ^{2} \theta \int_{0}^{\theta} \mathrm{e}^{\sin ^{2} w} \cos ^{5} w d w+2 I_{2} \int_{0}^{\theta} \mathrm{e}^{\sin ^{2} w} \cos ^{5} w d w-2 I_{3} \cos ^{4} \theta \int_{0}^{\theta} \mathrm{e}^{\sin ^{2} w} \cos ^{3} w d w \\
& +2 I_{1} \int_{0}^{\theta} \mathrm{e}^{\sin ^{2} w} \cos w d w-I_{2} \int_{0}^{\theta} \mathrm{e}^{\sin ^{2} w} \cos ^{3} w d w+I_{3} \cos ^{2} \theta \int_{0}^{\theta} \mathrm{e}^{\sin ^{2} w} \cos w d w \\
& +2 I_{1} \int_{0}^{\theta} \mathrm{e}^{\sin ^{2} w} \cos ^{3} w d w+I_{3} \cos ^{2} \theta \int_{0}^{\theta} \mathrm{e}^{\sin ^{2} w} \cos ^{3} w d w \\
& \left.-I_{2} \int_{0}^{\theta} \mathrm{e}^{\sin ^{2} w} \cos w d w+4 I_{3} \cos ^{4} \theta \int_{0}^{\theta} \mathrm{e}^{\sin ^{2} w} \cos ^{5} w d w\right) \frac{1}{2 I_{1}-I_{2}}, \\
s_{2,31}(\theta)= & -\left(2 \int_{0}^{\theta} \mathrm{e}^{\sin ^{2} w} \sin ^{3} w \cos ^{2} w d w+\int_{0}^{\theta} \mathrm{e}^{\sin ^{2} w} \sin ^{3} w d w+4\left(\int_{0}^{\theta} \mathrm{e}^{\sin ^{2} w} \sin ^{3} w \cos ^{2} w d w\right) \cos ^{2} \theta\right. \\
& \left.+2\left(\int_{0}^{\theta} \mathrm{e}^{\sin ^{2} w} \sin ^{3} w d w\right) \cos ^{2} \theta\right) \mathrm{e}^{2} \sin ^{2} \theta \cos ^{2} \sin \theta, \\
s_{2,32}(\theta)= & -\left(2 \int_{0}^{\theta} \mathrm{e}^{\sin ^{2} w} \cos ^{4} w \sin w d w+4\left(\int_{0}^{\theta} \mathrm{e}^{\sin ^{2} w} \cos ^{4} w \sin w d w\right) \cos ^{2} \theta\right. \\
& \left.+\int_{0}^{\theta} \mathrm{e}^{\sin ^{2} w} \cos ^{2} w \sin w d w+2\left(\int_{0}^{\theta} \mathrm{e}^{\sin ^{2} w} \cos ^{2} w \sin w d w\right) \cos ^{2} \theta\right) \mathrm{e}^{2 \sin ^{2} \theta} \cos \theta \sin \theta .
\end{aligned}
$$

We also have

$$
\begin{aligned}
s_{1}(\theta)= & s_{1,1}(\theta) a_{03} c_{10}+s_{1,2}(\theta) a_{01} c_{03}+s_{1,3}(\theta) a_{03} c_{01}+s_{1,4}(\theta) a_{01} c_{30}+s_{1,5}(\theta) c_{10} c_{21}+s_{1,6}(\theta) a_{01} a_{12} \\
& +s_{1,7}(\theta) c_{01} c_{12}+s_{1,8}(\theta) c_{01} a_{11}+s_{1,9}(\theta) c_{03} c_{10}+s_{1,10}(\theta) a_{12} c_{10}+s_{1,11}(\theta) c_{01} c_{21} \\
& +s_{1,12}(\theta) c_{10} c_{12}+s_{1,13}(\theta) a_{21} c_{10}+s_{1,14}(\theta) a_{01} a_{21}+s_{1,15}(\theta) c_{10} c_{30}+s_{1,16}(\theta) a_{01} c_{21} \\
& +s_{1,17}(\theta) a_{01} a_{03}+s_{1,18}(\theta) a_{01} c_{12}+s_{1,19}(\theta) a_{12} c_{01}+s_{1,20}(\theta) c_{01} c_{03}+s_{1,21}(\theta) a_{21} c_{01}
\end{aligned}
$$

and $s_{1, i}(\theta)$ for $i=1 \cdots, 21$ are given in the following expressions:

$$
\begin{aligned}
s_{1,1}(\theta)= & -\frac{1}{12}\left(24\left(\int_{0}^{\theta} \mathrm{e}^{2 \sin ^{2} w} \sin w \cos w d w\right) \cos ^{4} \theta-\mathrm{e}^{2 \sin ^{2} \theta}+12 \int_{0}^{\theta} \mathrm{e}^{2 \sin ^{2} w} \sin w \cos w d w\right. \\
& +4 \mathrm{e}^{2 \sin ^{2} \theta} \cos ^{2} \theta-36\left(\int_{0}^{\theta} \mathrm{e}^{2 \sin ^{2} w} \sin w \cos w d w\right) \cos ^{2} \theta-72\left(\int_{0}^{\theta} \mathrm{e}^{2 \sin ^{2} w} \cos ^{3} w \sin w d w\right) \cos ^{2} \theta \\
& +24 \int_{0}^{\theta} \mathrm{e}^{2 \sin ^{2} w} \cos ^{3} w \sin w d w+8 \mathrm{e}^{2 \sin ^{2} \theta} \cos ^{8} \theta+48\left(\int_{0}^{\theta} \mathrm{e}^{2 \sin ^{2} w} \cos ^{3} w \sin w d w\right) \cos ^{4} \theta \\
& \left.+3 \mathrm{e}^{2 \sin ^{2} \theta} \cos ^{4} \theta-14 \mathrm{e}^{2 \sin ^{2} \theta} \cos ^{6} \theta\right) \cos \theta \sin \theta
\end{aligned}
$$




$$
\begin{aligned}
& s_{1,2}(\theta)=-\frac{1}{3} \mathrm{e}^{2 \sin ^{2} \theta} \cos ^{6} \theta+2 \cos ^{6} \theta \int_{0}^{\theta} \mathrm{e}^{2 \sin ^{2} w} \sin w \cos w d w-\frac{2}{3} \mathrm{e}^{2 \sin ^{2} \theta} \cos ^{10} \theta \\
& -2\left(\int_{0}^{\theta} \mathrm{e}^{2 \sin ^{2} w} \cos ^{3} w \sin w d w\right) \cos ^{4} \theta-\int_{0}^{\theta} \mathrm{e}^{2 \sin ^{2} w} \sin w \cos w d w \\
& +\left(\int_{0}^{\theta} \mathrm{e}^{2 \sin ^{2} w} \sin w \cos w d w\right) \cos ^{4} \theta+\frac{7}{3} \mathrm{e}^{2 \sin ^{2} \theta} \cos ^{4} \theta-4 \cos ^{6} \theta \int_{0}^{\theta} \mathrm{e}^{2 \sin ^{2} w} \cos ^{3} w \sin w d w \\
& -\mathrm{e}^{2 \sin ^{2} \theta} \cos ^{2} \theta-\frac{1}{3} \mathrm{e}^{2 \sin ^{2} \theta} \cos ^{8} \theta+2 \int_{0}^{\theta} \mathrm{e}^{2 \sin ^{2} w} \cos ^{3} w \sin w d w \\
& s_{1,3}(\theta)=\mathrm{e}^{2 \sin ^{2} \theta}(\cos \theta-1)(\cos \theta+1)\left(-8 \cos ^{8} \theta I_{3}+14 \cos ^{6} \theta I_{3}-7 \cos ^{4} \theta I_{3}+8 \cos ^{4} \theta I_{1}\right. \\
& -4 \cos ^{4} \theta I_{2}+24 I_{3} \cos ^{3} \theta \sin \theta \mathrm{e}^{-2 \sin ^{2} \theta} \int_{0}^{\theta} \mathrm{e}^{2 \sin ^{2} w} \cos ^{2} w d w \\
& +48 I_{1} \cos ^{3} \theta \sin \theta \mathrm{e}^{-2 \sin ^{2} \theta} \int_{0}^{\theta} \mathrm{e}^{2 \sin ^{2} w} d w-48 I_{3} \cos ^{3} \theta \sin \theta \mathrm{e}^{-2 \sin ^{2} \theta} \int_{0}^{\theta} \mathrm{e}^{2 \sin ^{2} w} \cos ^{4} w d w \\
& -24 I_{2} \cos ^{3} \theta \sin \theta \mathrm{e}^{-2 \sin ^{2} \theta} \int_{0}^{\theta} \mathrm{e}^{2 \sin ^{2} w} d w+\cos ^{2} \theta I_{3}-10 \cos ^{2} \theta I_{1}+5 \cos ^{2} \theta I_{2} \\
& +12 I_{2} \cos \theta \sin \theta \mathrm{e}^{-2 \sin ^{2} \theta} \int_{0}^{\theta} \mathrm{e}^{2 \sin ^{2} w} d w-12 I_{3} \cos \theta \sin \theta \mathrm{e}^{-2 \sin ^{2} \theta} \int_{0}^{\theta} \mathrm{e}^{2 \sin ^{2} w} \cos ^{2} w d w \\
& -24 I_{1} \cos \theta \sin \theta \mathrm{e}^{-2 \sin ^{2} \theta} \int_{0}^{\theta} \mathrm{e}^{2 \sin ^{2} w} d w \\
& \left.+24 I_{3} \cos \theta \sin \theta \mathrm{e}^{-2 \sin ^{2} \theta} \int_{0}^{\theta} \mathrm{e}^{2 \sin ^{2} w} \cos ^{4} w d w-I_{2}+2 I_{1}\right) \frac{1}{12\left(I_{2}-2 I_{1}\right)}, \\
& s_{1,4}(\theta)=-\frac{1}{12}\left(24 \cos ^{4} \theta \mathrm{e}^{-2 \sin ^{2} \theta} \int_{0}^{\theta} \mathrm{e}^{2 \sin ^{2} w} \sin w \cos w d w-7+3 \cos ^{4} \theta\right. \\
& -8 \cos ^{8} \theta+14 \cos ^{2} \theta-24 \cos ^{2} \theta \mathrm{e}^{-2 \sin ^{2} \theta} \int_{0}^{\theta} \mathrm{e}^{2 \sin ^{2} w} \cos ^{3} w \sin w d w \\
& -2 \cos ^{6} \theta+12 \cos ^{2} \theta \mathrm{e}^{-2 \sin ^{2} \theta} \int_{0}^{\theta} \mathrm{e}^{2 \sin ^{2} w} \sin w \cos w d w \\
& \left.-48 \cos ^{4} \theta \mathrm{e}^{-2 \sin ^{2} \theta} \int_{0}^{\theta} \mathrm{e}^{2 \sin ^{2} w} \cos ^{3} w \sin w d w\right) \mathrm{e}^{2 \sin ^{2} \theta} \sin \theta \cos \theta, \\
& s_{1,5}(\theta)=-\frac{1}{3}\left[-\mathrm{e}^{2 \sin ^{2} \theta} \cos ^{2} \theta+12\left(\int_{0}^{\theta} \mathrm{e}^{2 \sin ^{2} w} \sin w \cos w d w\right) \cos ^{4} \theta\right. \\
& +24\left(\int_{0}^{\theta} \mathrm{e}^{2 \sin ^{2} w} \cos ^{3} w \sin w d w\right) \cos ^{4} \theta-3 \int_{0}^{\theta} \mathrm{e}^{2 \sin ^{2} w} \sin w \cos w d w \\
& -6\left(\int_{0}^{\theta} \mathrm{e}^{2 \sin ^{2} w} \sin w \cos w d w\right) \cos ^{2} \theta-6 \int_{0}^{\theta} \mathrm{e}^{2 \sin ^{2} w} \cos ^{3} w \sin w d w \\
& -12\left(\int_{0}^{\theta} \mathrm{e}^{2} \sin ^{2} w \cos ^{3} w \sin w d w\right) \cos ^{2} \theta+4 \mathrm{e}^{2 \sin ^{2} \theta} \cos ^{8} \theta \\
& \left.-3 \mathrm{e}^{2 \sin ^{2} \theta} \cos ^{4} \theta\right] \cos ^{2} \theta
\end{aligned}
$$




$$
\begin{aligned}
& s_{1,6}(\theta)=-\frac{1}{3}\left(12 \int_{0}^{\theta} \mathrm{e}^{2 \sin ^{2} w} \cos ^{3} w \sin w d w \cos ^{4} \theta+9\left(\int_{0}^{\theta} \mathrm{e}^{2 \sin ^{2} w} \sin w \cos w d w\right) \cos ^{2} \theta\right. \\
& +6 \int_{0}^{\theta} \mathrm{e}^{2 \sin ^{2} w} \cos ^{3} w \sin w d w-3 \int_{0}^{\theta} \mathrm{e}^{2 \sin ^{2} w} \sin w \cos w d w+4 \mathrm{e}^{2 \sin ^{2} \theta} \cos ^{4} \theta \\
& -6\left(\int_{0}^{\theta} \mathrm{e}^{2 \sin ^{2} w} \sin w \cos w d w\right) \cos ^{4} \theta-5 \mathrm{e}^{2 \sin ^{2} \theta} \cos ^{6} \theta+2 \mathrm{e}^{2 \sin ^{2} \theta} \cos ^{8} \theta \\
& \left.-18\left(\int_{0}^{\theta} \mathrm{e}^{2 \sin ^{2} w} \cos ^{3} w \sin w d w\right) \cos ^{2} \theta-\mathrm{e}^{2 \sin ^{2} \theta} \cos ^{2} \theta\right) \cos ^{2} \theta, \\
& s_{1,7}(\theta)=-\frac{1}{12} \mathrm{e}^{2 \sin ^{2} \theta}\left(12 I_{2} \cos \theta \sin \theta \mathrm{e}^{-2 \sin ^{2} \theta} \int_{0}^{\theta} \mathrm{e}^{2 \sin ^{2} w} d w\right. \\
& -12 I_{3} \cos ^{3} \theta \sin \theta \mathrm{e}^{-2 \sin ^{2} \theta} \int_{0}^{\theta} \mathrm{e}^{2 \sin ^{2} w} \cos ^{2} w d w-24 I_{1} \cos \theta \sin \theta \mathrm{e}^{-2 \sin ^{2} \theta} \int_{0}^{\theta} \mathrm{e}^{2 \sin ^{2} w} d w \\
& +12 I_{2} \cos ^{3} \theta \sin \theta \mathrm{e}^{-2 \sin ^{2} \theta} \int_{0}^{\theta} \mathrm{e}^{2 \sin ^{2} w} d w+24 I_{3} \cos ^{3} \theta \sin \theta \mathrm{e}^{-2 \sin ^{2} \theta} \int_{0}^{\theta} \mathrm{e}^{2 \sin ^{2} w} \cos ^{4} w d w \\
& +24 I_{3} \cos \theta \sin \theta \mathrm{e}^{-2 \sin ^{2} \theta} \int_{0}^{\theta} \mathrm{e}^{2 \sin ^{2} w} \cos ^{4} w d w-12 I_{3} \cos \theta \sin \theta \mathrm{e}^{-2 \sin ^{2} \theta} \int_{0}^{\theta} \mathrm{e}^{2 \sin ^{2} w} \cos ^{2} w d w \\
& -24 I_{1} \cos ^{3} \theta \sin \theta \mathrm{e}^{-2 \sin ^{2} \theta} \int_{0}^{\theta} \mathrm{e}^{2 \sin ^{2} w} d w+24 I_{3} \cos ^{5} \theta \sin \theta \mathrm{e}^{-2 \sin ^{2} \theta} \int_{0}^{\theta} \mathrm{e}^{2 \sin ^{2} w}\left(\cos ^{4} w\right)^{2} d w \\
& +48 I_{1} \cos ^{5} \theta \sin \theta \mathrm{e}^{-2 \sin ^{2} \theta} \int_{0}^{\theta} \mathrm{e}^{2 \sin ^{2} w} d w-24 I_{2} \cos ^{5} \theta \sin \theta \mathrm{e}^{-2 \sin ^{2} \theta} \int_{0}^{\theta} \mathrm{e}^{2 \sin ^{2} w} d w \\
& -48 I_{3} \cos ^{5} \theta \sin \theta \mathrm{e}^{-2 \sin ^{2} \theta} \int_{0}^{\theta} \mathrm{e}^{2 \sin ^{2} w} \cos ^{4} w d w-16 \cos ^{4} \theta I_{3}+9 \cos ^{6} \theta I_{3}+5 \cos ^{2} \theta I_{3} \\
& -12 \cos ^{2} \theta I_{1}-8 I_{3} \cos ^{10} \theta+8 \cos ^{6} \theta I_{1}-4 \cos \theta^{6} I_{2}-5 I_{2}+10 I_{1}+6 \cos ^{2} \theta I_{2} \\
& \left.-6 \cos ^{4} \theta I_{1}+3 \cos ^{4} \theta I_{2}+10 \cos ^{8} \theta I_{3}\right) \frac{1}{2 I_{1}-I_{2}}, \\
& s_{1,8}(\theta)=\frac{1}{12} \mathrm{e}^{2 \sin \theta^{2}}\left(-4 \cos ^{6} \theta I_{2}-48 I_{3} \cos ^{5} \theta \sin \theta \mathrm{e}^{-2 \sin ^{2} \theta} \int_{0}^{\theta} \mathrm{e}^{2 \sin ^{2} w} \cos ^{4} w d w\right. \\
& -3 \cos ^{4} \theta I_{2}+14 \cos ^{4} \theta I_{3}-24 I_{3} \cos ^{3} \theta \sin \theta \mathrm{e}^{-2 \sin ^{2} \theta} \int_{0}^{\theta} \mathrm{e}^{2 \sin ^{2} w} \cos ^{4} w d w \\
& +3 \cos ^{6} \theta I_{3}-2 \cos ^{8} \theta I_{3}+24 I_{3} \cos ^{5} \theta \sin \theta \mathrm{e}^{-2 \sin ^{2} \theta} \int_{0}^{\theta} \mathrm{e}^{2 \sin ^{2} w} \cos ^{2} w d w \\
& +6 \cos ^{4} \theta I_{1}+8 \cos ^{6} \theta I_{1}+7 I_{2}-24 I_{2} \cos ^{5} \theta \sin \theta \mathrm{e}^{-2 \sin ^{2} \theta} \int_{0}^{\theta} \mathrm{e}^{2 \sin ^{2} w} d w \\
& -12 I_{2} \cos ^{3} \theta \sin \theta \mathrm{e}^{-2 \sin ^{2} \theta} \int_{0}^{\theta} \mathrm{e}^{2 \sin ^{2} w} d w+48 I_{1} \cos ^{5} \theta \sin \theta \mathrm{e}^{-2 \sin ^{2} \theta} \int_{0}^{\theta} \mathrm{e}^{2 \sin ^{2} w} d w-8 I_{3} \cos ^{10} \theta \\
& -7 \cos ^{2} \theta I_{3}+12 I_{3} \cos ^{3} \theta \sin \theta \mathrm{e}^{-2 \sin ^{2} \theta} \int_{0}^{\theta} \mathrm{e}^{2 \sin ^{2} w} \cos ^{2} w d w \\
& \left.+24 I_{1} \cos ^{3} \theta \sin \theta \mathrm{e}^{-2 \sin ^{2} \theta} \int_{0}^{\theta} \mathrm{e}^{2 \sin ^{2} w} d w-14 I_{1}\right) \frac{1}{-I_{2}+2 I_{1}},
\end{aligned}
$$




$$
\begin{aligned}
& s_{1,9}(\theta)=-\left(\int_{0}^{\theta} \mathrm{e}^{2 \sin ^{2} w} \sin w \cos w d w\right) \cos ^{4} \theta+\int_{0}^{\theta} \mathrm{e}^{2 \sin ^{2} w} \sin w \cos w d w \\
& -\mathrm{e}^{2 \sin ^{2} \theta} \cos ^{8} \theta-2 \cos ^{6} \theta \int_{0}^{\theta} \mathrm{e}^{2 \sin ^{2} w} \sin w \cos w d w-\mathrm{e}^{2 \sin ^{2} \theta} \cos ^{6} \theta \\
& +5 / 3 \mathrm{e}^{2 \sin ^{2} \theta} \cos ^{4} \theta+2 \int_{0}^{\theta} \mathrm{e}^{2 \sin ^{2} w} \cos ^{3} w \sin w d w \\
& -4 \cos ^{6} \theta \int_{0}^{\theta} \mathrm{e}^{2 \sin ^{2} w} \cos ^{3} w \sin w d w-\frac{2}{3} \mathrm{e}^{2 \sin ^{2} \theta} \cos ^{10} \theta \\
& +\mathrm{e}^{2 \sin ^{2} \theta} \cos ^{2} \theta-2\left(\int_{0}^{\theta} \mathrm{e}^{2 \sin ^{2} w} \cos ^{3} w \sin w d w\right) \cos ^{4} \theta, \\
& s_{1,10}(\theta)=-\frac{1}{3}\left(\mathrm{e}^{2 \sin \theta^{2}} \cos ^{2} \theta+12\left(\int_{0}^{\theta} \mathrm{e}^{2 \sin ^{2} w} \cos ^{3} w \sin w d w\right) \cos ^{4} \theta-3 \mathrm{e}^{2 \sin ^{2} \theta} \cos ^{6} \theta\right. \\
& -9\left(\int_{0}^{\theta} \mathrm{e}^{2 \sin ^{2} w} \sin w \cos w d w\right) \cos ^{2} \theta+6\left(\int_{0}^{\theta} \mathrm{e}^{2 \sin ^{2} w} \sin w \cos w d w\right) \cos ^{4} \theta \\
& +6 \int_{0}^{\theta} \mathrm{e}^{2 \sin ^{2} w} \cos ^{3} w \sin w d w-18\left(\int_{0}^{\theta} \mathrm{e}^{2 \sin ^{2} w} \cos ^{3} w \sin w d w\right) \cos ^{2} \theta \\
& \left.+3 \int_{0}^{\theta} \mathrm{e}^{2 \sin ^{2} w} \sin w \cos w d w+2 \mathrm{e}^{2 \sin ^{2} \theta} \cos ^{8} \theta\right) \cos ^{2} \theta \\
& s_{1,11}(\theta)=-\frac{1}{3} \mathrm{e}^{2 \sin \theta^{2}} \cos ^{2} \theta\left(-6 I_{1} \mathrm{e}^{-2 \sin ^{2} \theta} \int_{0}^{\theta} \mathrm{e}^{2 \sin ^{2} w} d w+4 \cos ^{7} \theta \sin \theta I_{3}\right. \\
& -4 \cos ^{3} \theta \sin \theta I_{1}+6 I_{2} \cos ^{2} \theta \mathrm{e}^{-2 \sin ^{2} \theta} \int_{0}^{\theta} \mathrm{e}^{2 \sin ^{2} w} d w \\
& -12 I_{1} \cos ^{2} \theta \mathrm{e}^{-2 \sin ^{2} \theta} \int_{0}^{\theta} \mathrm{e}^{2 \sin ^{2} w} d w-3 I_{3} \mathrm{e}^{-2 \sin ^{2} \theta} \int_{0}^{\theta} \mathrm{e}^{2 \sin ^{2} w} \cos ^{2} w d w \\
& +2 \cos ^{3} \theta \sin \theta I_{2}+24 I_{1} \cos ^{4} \theta \mathrm{e}^{-2 \sin ^{2} \theta} \int_{0}^{\theta} \mathrm{e}^{2 \sin ^{2} w} d w+12 I_{3} \cos ^{2} \theta \mathrm{e}^{-2 \sin ^{2} \theta} \int_{0}^{\theta} \mathrm{e}^{2 \sin ^{2} w} \cos ^{4} w d w \\
& +12 I_{3} \cos ^{4} \theta \mathrm{e}^{-2 \sin ^{2} \theta} \int_{0}^{\theta} \mathrm{e}^{2 \sin ^{2} w} \cos ^{2} w d w-2 \cos \theta \sin \theta I_{1} \\
& -12 I_{2} \cos ^{4} \theta \mathrm{e}^{-2 \sin \theta^{2}} \int_{0}^{\theta} \mathrm{e}^{2 \sin ^{2} w} d w+\cos \theta \sin \theta I_{2} \\
& +6 I_{3} \mathrm{e}^{-2 \sin ^{2} \theta} \int_{0}^{\theta} \mathrm{e}^{2 \sin ^{2} w} \cos ^{4} w d w \\
& -24 I_{3} \cos ^{4} \theta \mathrm{e}^{-2 \sin \theta^{2}} \int_{0}^{\theta} \mathrm{e}^{2 \sin ^{2} w} \cos ^{4} w d w+3 I_{2} \mathrm{e}^{-2 \sin ^{2} \theta} \int_{0}^{\theta} \mathrm{e}^{2 \sin ^{2} w} d w \\
& \left.-\cos ^{3} \theta \sin \theta I_{3}-6 I_{3} \cos ^{2} \theta \mathrm{e}^{-2 \sin ^{2} \theta} \int_{0}^{\theta} \mathrm{e}^{2 \sin ^{2} w} \cos ^{2} w d w\right) \frac{1}{2 I_{1}-I_{2}},
\end{aligned}
$$




$$
\begin{aligned}
& s_{1,12}(\theta)=-\frac{1}{12}\left(-12 \mathrm{e}^{-2 \sin ^{2} \theta} \int_{0}^{\theta} \mathrm{e}^{2 \sin ^{2} w} \sin w \cos w d w-15 \cos ^{4} \theta-2 \cos ^{6} \theta\right. \\
& +24 \cos ^{4} \theta \mathrm{e}^{-2 \sin ^{2} \theta} \int_{0}^{\theta} \mathrm{e}^{2 \sin ^{2} w} \sin w \cos w d w-24 \cos ^{2} \theta \mathrm{e}^{-2 \sin ^{2} \theta} \int_{0}^{\theta} \mathrm{e}^{2 \sin ^{2} w} \cos ^{3} w \sin w d w \\
& -24 \mathrm{e}^{-2 \sin ^{2} \theta} \int_{0}^{\theta} \mathrm{e}^{2 \sin ^{2} w} \cos ^{3} w \sin w d w+8 \cos ^{8} \theta+4 \cos ^{2} \theta \\
& +48 \cos ^{4} \theta \mathrm{e}^{-2 \sin \theta^{2}} \int_{0}^{\theta} \mathrm{e}^{2 \sin ^{2} w} \cos ^{3} w \sin w d w \\
& \left.-12 \cos ^{2} \theta \mathrm{e}^{-2 \sin ^{2} \theta} \int_{0}^{\theta} \mathrm{e}^{2 \sin ^{2} w} \sin w \cos w d w+5\right) \mathrm{e}^{2 \sin ^{2} \theta} \cos \theta \sin \theta, \\
& s_{1,13}(\theta)=\frac{1}{12}\left(-12 \cos ^{2} \theta \mathrm{e}^{-2 \sin ^{2} \theta} \int_{0}^{\theta} \mathrm{e}^{2 \sin ^{2} w} \sin w \cos w d w\right. \\
& +24 \cos ^{4} \theta \mathrm{e}^{-2 \sin ^{2} \theta} \int_{0}^{\theta} \mathrm{e}^{2 \sin ^{2} w} \sin w \cos w d w+8 \cos ^{8} \theta-3 \cos ^{4} \theta \\
& -24 \cos ^{2} \theta \mathrm{e}^{-2 \sin ^{2} \theta} \int_{0}^{\theta} \mathrm{e}^{2 \sin ^{2} w} \cos ^{3} w \sin w d w+48 \cos ^{4} \theta \mathrm{e}^{-2 \sin ^{2} \theta} \int_{0}^{\theta} \mathrm{e}^{2 \sin ^{2} w} \cos ^{3} w \sin w d w \\
& \left.-1-2 \cos ^{2} \theta-2 \cos ^{6} \theta\right) \mathrm{e}^{2 \sin ^{2} \theta} \cos \theta \sin \theta, \\
& s_{1,14}(\theta)=\frac{1}{12}\left(1+48 \cos ^{4} \theta \mathrm{e}^{-2 \sin ^{2} \theta} \int_{0}^{\theta} \mathrm{e}^{2 \sin ^{2} w} \cos ^{3} w \sin w d w+8 \cos ^{8} \theta\right. \\
& -24 \cos ^{2} \theta \mathrm{e}^{-2 \sin ^{2} \theta} \int_{0}^{\theta} \mathrm{e}^{2 \sin ^{2} w} \cos ^{3} w \sin w d w-10 \cos ^{6} \theta \\
& -24 \cos \theta^{4} \mathrm{e}^{-2 \sin ^{2} \theta} \int_{0}^{\theta} \mathrm{e}^{2 \sin ^{2} w} \sin w \cos w d w-2 \cos ^{2} \theta \\
& \left.+12 \cos ^{2} \theta \mathrm{e}^{-2 \sin ^{2} \theta} \int_{0}^{\theta} \mathrm{e}^{2 \sin ^{2} w} \sin w \cos w d w+3 \cos ^{4} \theta\right) \mathrm{e}^{2 \sin ^{2} \theta} \cos \theta \sin \theta, \\
& s_{1,15}(\theta)=\frac{1}{12}\left(24 \cos ^{2} \theta \mathrm{e}^{-2 \sin ^{2} \theta} \int_{0}^{\theta} \mathrm{e}^{2 \sin ^{2} w} \cos ^{3} w \sin w d w+10 \cos ^{6} \theta\right. \\
& +24 \cos ^{4} \theta \mathrm{e}^{-2 \sin ^{2} \theta} \int_{0}^{\theta} \mathrm{e}^{2 \sin ^{2} w} \sin w \cos w d w \\
& +48 \cos ^{4} \theta \mathrm{e}^{-2 \sin ^{2} \theta} \int_{0}^{\theta} \mathrm{e}^{2 \sin ^{2} w} \cos ^{3} w \sin w d w \\
& +3 \cos ^{4} \theta+12 \cos ^{2} \theta \mathrm{e}^{-2 \sin ^{2} \theta} \int_{0}^{\theta} \mathrm{e}^{2 \sin ^{2} w} \sin w \cos w d w+8 \cos ^{8} \theta \\
& \left.-14 \cos ^{2} \theta-7\right) \mathrm{e}^{2 \sin ^{2} \theta} \cos \theta \sin \theta \text {, }
\end{aligned}
$$




$$
\begin{aligned}
& s_{1,16}(\theta)=-\frac{1}{3}\left(-6 \int_{0}^{\theta} \mathrm{e}^{2 \sin ^{2} w} \cos ^{3} w \sin w d w-12\left(\int_{0}^{\theta} \mathrm{e}^{2 \sin ^{2} w} \sin w \cos w d w\right) \cos ^{4} \theta\right. \\
& +\mathrm{e}^{2 \sin ^{2} \theta} \cos ^{2} \theta+4 \mathrm{e}^{2 \sin ^{2} \theta} \cos ^{8} \theta-12\left(\int_{0}^{\theta} \mathrm{e}^{2 \sin ^{2} w} \cos ^{3} w \sin w d w\right) \cos ^{2} \theta \\
& +24\left(\int_{0}^{\theta} \mathrm{e}^{2 \sin ^{2} w} \cos ^{3} w \sin w d w\right) \cos ^{4} \theta-4 \mathrm{e}^{2 \sin ^{2} \theta} \cos ^{6} \theta \\
& +6\left(\int_{0}^{\theta} \mathrm{e}^{2 \sin ^{2} w} \sin w \cos w d w\right) \cos ^{2} \theta-\mathrm{e}^{2 \sin ^{2} \theta} \cos ^{4} \theta \\
& \left.+3 \int_{0}^{\theta} \mathrm{e}^{2 \sin ^{2} w} \sin w \cos w d w\right) \cos ^{2} \theta \\
& s_{1,17}(\theta)=-\frac{1}{12}\left(48 \cos ^{4} \theta \mathrm{e}^{-2 \sin ^{2} \theta} \int_{0}^{\theta} \mathrm{e}^{2 \sin ^{2} w} \cos ^{3} w \sin w d w\right. \\
& -12 \mathrm{e}^{-2 \sin ^{2} \theta} \int_{0}^{\theta} \mathrm{e}^{2 \sin ^{2} w} \sin w \cos w d w-24 \cos ^{4} \theta \mathrm{e}^{-2 \sin ^{2} \theta} \int_{0}^{\theta} \mathrm{e}^{2 \sin ^{2} w} \sin w \cos w d w \\
& +36 \cos ^{2} \theta \mathrm{e}^{-2 \sin \theta^{2}} \int_{0}^{\theta} \mathrm{e}^{2 \sin ^{2} w} \sin w \cos w d w+8 \cos ^{8} \theta+1+21 \cos ^{4} \theta \\
& +24 \mathrm{e}^{-2 \sin ^{2} \theta} \int_{0}^{\theta} \mathrm{e}^{2 \sin ^{2} w} \cos ^{3} w \sin w d w-72 \cos ^{2} \theta \mathrm{e}^{-2 \sin ^{2} \theta} \int_{0}^{\theta} \mathrm{e}^{2 \sin ^{2} w} \cos ^{3} w \sin w d w-8 \cos ^{2} \theta \\
& \left.-22 \cos ^{6} \theta\right) \mathrm{e}^{2 \sin \theta^{2}} \cos \theta \sin \theta, \\
& s_{1,18}(\theta)=-\frac{1}{12}\left(-24 \cos ^{4} \theta \mathrm{e}^{-2 \sin ^{2} \theta} \int_{0}^{\theta} \mathrm{e}^{2 \sin ^{2} w} \sin w \cos w d w\right. \\
& -24 \mathrm{e}^{-2 \sin ^{2} \theta} \int_{0}^{\theta} \mathrm{e}^{2 \sin ^{2} w} \cos ^{3} w \sin w d w+16 \cos ^{2} \theta-5 \\
& +12 \cos ^{2} \theta \mathrm{e}^{-2 \sin ^{2} \theta} \int_{0}^{\theta} \mathrm{e}^{2 \sin ^{2} w} \sin w \cos w d w \\
& -9 \cos ^{4} \theta-24 \cos ^{2} \theta \mathrm{e}^{-2 \sin ^{2} \theta} \int_{0}^{\theta} \mathrm{e}^{2 \sin ^{2} w} \cos ^{3} w \sin w d w-10 \cos ^{6} \theta \\
& +12 \mathrm{e}^{-2 \sin ^{2} \theta} \int_{0}^{\theta} \mathrm{e}^{2 \sin ^{2} w} \sin w \cos w d w+8 \cos ^{8} \theta \\
& \left.+48 \cos ^{4} \theta \mathrm{e}^{-2 \sin ^{2} \theta} \int_{0}^{\theta} \mathrm{e}^{2 \sin ^{2} w} \cos ^{3} w \sin w d w\right) \mathrm{e}^{2 \sin ^{2} \theta} \cos \theta \sin \theta,
\end{aligned}
$$




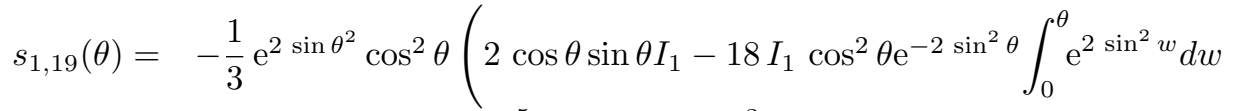

$$
\begin{aligned}
& -\cos \theta \sin \theta I_{2}-3 \cos ^{5} \theta \sin \theta I_{3}+\cos ^{3} \theta \sin \theta I_{2} \\
& +9 I_{2} \cos ^{2} \theta \mathrm{e}^{-2 \sin ^{2} \theta} \int_{0}^{\theta} \mathrm{e}^{2 \sin ^{2} w} d w+18 I_{3} \cos ^{2} \theta \mathrm{e}^{-2 \sin ^{2} \theta} \int_{0}^{\theta} \mathrm{e}^{2 \sin ^{2} w} \cos ^{4} w d w \\
& +\cos ^{3} \theta \sin \theta I_{3}-6 I_{2} \cos ^{4} \theta \mathrm{e}^{-2 \sin ^{2} \theta} \int_{0}^{\theta} \mathrm{e}^{2 \sin ^{2} w} d w \\
& +6 I_{3} \cos ^{4} \theta \mathrm{e}^{-2 \sin ^{2} \theta} \int_{0}^{\theta} \mathrm{e}^{2 \sin ^{2} w} \cos ^{2} w d w+6 I_{1} \mathrm{e}^{-2 \sin ^{2} \theta} \int_{0}^{\theta} \mathrm{e}^{2 \sin ^{2} w} d w \\
& +2 \cos \theta^{7} \sin \theta I_{3}-3 I_{2} \mathrm{e}^{-2 \sin ^{2} \theta} \int_{0}^{\theta} \mathrm{e}^{2 \sin ^{2} w} d w-9 I_{3} \cos ^{2} \theta \mathrm{e}^{-2 \sin ^{2} \theta} \int_{0}^{\theta} \mathrm{e}^{2 \sin ^{2} w} \cos ^{2} w d w \\
& -12 I_{3} \cos ^{4} \theta \mathrm{e}^{-2 \sin ^{2} \theta} \int_{0}^{\theta} \mathrm{e}^{2 \sin ^{2} w} \cos ^{4} w d w+12 I_{1} \cos ^{4} \theta \mathrm{e}^{-2 \sin ^{2} \theta} \int_{0}^{\theta} \mathrm{e}^{2} \sin ^{2} w d w \\
& \left.-2 \cos ^{3} \theta \sin \theta I_{1}-6 I_{3} \mathrm{e}^{-2 \sin ^{2} \theta} \int_{0}^{\theta} \mathrm{e}^{2 \sin ^{2} w} \cos ^{4} w d w+3 I_{3} \mathrm{e}^{-2 \sin ^{2} \theta} \int_{0}^{\theta} \mathrm{e}^{2 \sin ^{2} w} \cos ^{2} w d w\right) \frac{1}{2 I_{1}-I_{2}}, \\
& s_{1,20}(\theta)=-\frac{1}{3} \mathrm{e}^{2 \sin ^{2} \theta}\left(-6 I_{3} \cos ^{4} \theta \mathrm{e}^{-2 \sin ^{2} \theta} \int_{0}^{\theta} \mathrm{e}^{2 \sin ^{2} w} \cos ^{4} w d w\right. \\
& +2 \cos ^{3} \theta \sin \theta I_{2}+12 I_{1} \cos ^{6} \theta \mathrm{e}^{-2 \sin ^{2} \theta} \int_{0}^{\theta} \mathrm{e}^{2 \sin ^{2} w} d w \\
& -6 I_{2} \cos ^{6} \theta \mathrm{e}^{-2 \sin ^{2} \theta} \int_{0}^{\theta} \mathrm{e}^{2 \sin ^{2} w} d w-3 I_{2} \cos ^{4} \theta \mathrm{e}^{-2 \sin ^{2} \theta} \int_{0}^{\theta} \mathrm{e}^{2 \sin ^{2} w} d w \\
& -3 \cos ^{3} \theta \sin \theta I_{3}-12 I_{3} \cos ^{6} \theta \mathrm{e}^{-2 \sin \theta^{2}} \int_{0}^{\theta} \mathrm{e}^{2 \sin ^{2} w} \cos ^{4} w d w \\
& +6 I_{1} \cos ^{4} \theta \mathrm{e}^{-2 \sin ^{2} \theta} \int_{0}^{\theta} \mathrm{e}^{2 \sin ^{2} w} d w-6 I_{1} \mathrm{e}^{-2 \sin ^{2} \theta} \int_{0}^{\theta} \mathrm{e}^{2 \sin ^{2} w} d w+\cos ^{5} \theta \sin \theta I_{2} \\
& +4 \cos ^{5} \theta \sin \theta I_{3}+3 \cos \theta \sin \theta I_{2}+3 \cos ^{7} \theta \sin \theta I_{3} \\
& -3 I_{3} \mathrm{e}^{-2 \sin ^{2} \theta} \int_{0}^{\theta} \mathrm{e}^{2 \sin ^{2} w} \cos ^{2} w d w+3 I_{3} \cos ^{4} \theta \mathrm{e}^{-2 \sin ^{2} \theta} \int_{0}^{\theta} \mathrm{e}^{2 \sin ^{2} w} \cos ^{2} w d w \\
& +3 I_{2} \mathrm{e}^{-2 \sin ^{2} \theta} \int_{0}^{\theta} \mathrm{e}^{2 \sin ^{2} w} d w-6 \cos \theta \sin \theta I_{1}-2 \cos ^{5} \theta \sin \theta I_{1} \\
& +6 I_{3} \mathrm{e}^{-2 \sin ^{2} \theta} \int_{0}^{\theta} \mathrm{e}^{2 \sin ^{2} w} \cos ^{4} w d w-4 \cos ^{3} \theta \sin \theta I_{1}+2 I_{3} \cos ^{9} \theta \sin \theta \\
& \left.+6 I_{3} \cos ^{6} \theta \mathrm{e}^{-2 \sin ^{2} \theta} \int_{0}^{\theta} \mathrm{e}^{2 \sin ^{2} w} \cos ^{2} w d w\right) \frac{1}{2 I_{1}-I_{2}},
\end{aligned}
$$




$$
\begin{aligned}
s_{1,21}(\theta)= & \frac{1}{12} \mathrm{e}^{2 \sin \theta^{2}}\left(2 \cos ^{4} \theta I_{3}-48 I_{3} \cos ^{5} \theta \sin \theta \mathrm{e}^{-2 \sin ^{2} \theta} \int_{0}^{\theta} \mathrm{e}^{2 \sin ^{2} w} \cos ^{4} w d w\right. \\
& -3 \cos ^{6} \theta I_{3}-4 \cos ^{6} \theta I_{2}+I_{2}-12 I_{3} \cos ^{3} \theta \sin \theta \mathrm{e}^{-2 \sin \theta^{2}} \int_{0}^{\theta} \mathrm{e}^{2 \sin ^{2} w} \cos ^{2} w d w \\
& -6 \cos ^{4} \theta I_{1}-8 I_{3} \cos ^{10} \theta-2 I_{1}+24 I_{3} \cos ^{3} \theta \sin \theta \mathrm{e}^{-2 \sin ^{2} \theta} \int_{0}^{\theta} \mathrm{e}^{2 \sin ^{2} w} \cos ^{4} w d w \\
& -24 I_{2} \cos ^{5} \theta \sin \theta \mathrm{e}^{-2 \sin \theta^{2}} \int_{0}^{\theta} \mathrm{e}^{2 \sin ^{2} w} d w+12 I_{2} \cos ^{3} \theta \sin \theta \mathrm{e}^{-2 \sin ^{2} \theta} \int_{0}^{\theta} \mathrm{e}^{2 \sin ^{2} w} d w \\
& -24 I_{1} \cos ^{3} \theta \sin \theta \mathrm{e}^{-2 \sin ^{2} \theta} \int_{0}^{\theta} \mathrm{e}^{2 \sin ^{2} w} d w+10 \cos ^{8} \theta I_{3} \\
& +8 \cos ^{6} \theta I_{1}-\cos ^{2} \theta I_{3}+24 I_{3} \cos ^{5} \theta \sin \theta \mathrm{e}^{-2 \sin ^{2} \theta} \int_{0}^{\theta} \mathrm{e}^{2 \sin ^{2} w} \cos ^{2} w d w \\
& \left.+3 \cos ^{4} \theta I_{2}+48 I_{1} \cos ^{5} \theta \sin \theta \mathrm{e}^{-2 \sin ^{2} \theta} \int_{0}^{\theta} \mathrm{e}^{2 \sin ^{2} w} d w\right) \frac{1}{2 I_{1}-I_{2}} .
\end{aligned}
$$

Now we present the expression of $s_{0}(\theta)$.

$$
\begin{aligned}
s_{0}(\theta)= & s_{0,1}(\theta) a_{12} c_{11}+s_{0,2}(\theta) a_{20} c_{03}+s_{0,3}(\theta) a_{20} c_{21}+s_{0,4}(\theta) a_{21} c_{11}+s_{0,5}(\theta) a_{21} c_{02}+s_{0,6}(\theta) a_{21} c_{20} \\
& +s_{0,7}(\theta) c_{02} c_{30}+s_{0,8}(\theta) a_{02} a_{12}+s_{0,9}(\theta) a_{02} c_{03}+s_{0,10}(\theta) a_{02} c_{21}+s_{0,11}(\theta) a_{03} a_{11}+s_{0,12}(\theta) a_{03} c_{02} \\
& +s_{0,13}(\theta) a_{03} c_{20}+s_{0,14}(\theta) a_{11} a_{21}+s_{0,15}(\theta) a_{11} c_{12}+s_{0,16}(\theta) a_{11} c_{30}+s_{0,17}(\theta) a_{12} a_{20} \\
& +s_{0,18}(\theta) a_{11} a_{12}+s_{0,19}(\theta) a_{03} a_{20}+s_{0,20}(\theta) a_{02} a_{21}+s_{0,21}(\theta) a_{11} c_{21}+s_{0,22}(\theta) c_{20} c_{30} \\
& +s_{0,23}(\theta) c_{11} c_{03}+s_{0,24}(\theta) c_{02} c_{03}+s_{0,25}(\theta) c_{11} c_{21}+s_{0,26}(\theta) c_{12} c_{20}+s_{0,27}(\theta) c_{02} c_{12}+s_{0,28}(\theta) a_{02} c_{12} \\
& +s_{0,29} a_{02} c_{30}+s_{0,30}(\theta) a_{02} a_{03}+s_{0,31}(\theta) a_{20} a_{21}+s_{0,32}(\theta) c_{11} c_{30}+s_{0,33}(\theta) c_{20} c_{21}+s_{0,34}(\theta) c_{02} c_{21} \\
& +s_{0,35}(\theta) c_{03} c_{20}+s_{0,36}(\theta) c_{11} c_{12}+s_{0,37}(\theta) a_{20} c_{30}+s_{0,38}(\theta) a_{12} c_{20}+s_{0,39}(\theta) a_{20} c_{12} \\
& +s_{0,40}(\theta) a_{11} c_{03}+s_{0,41}(\theta) a_{12} c_{02}+s_{0,42}(\theta) a_{03} c_{11} .
\end{aligned}
$$

and $s_{0, i}(\theta)$ for $i=1 \cdots, 42$ are the following:

$$
\begin{aligned}
s_{0,1}(\theta)= & -\cos ^{2} \theta\left(2 \cos ^{4} \theta \int_{0}^{\theta} \mathrm{e}^{\sin ^{2} w} \cos w d w+\int_{0}^{\theta} \mathrm{e}^{\sin ^{2} w} \cos w d w-3 \cos ^{2} \theta \int_{0}^{\theta} \mathrm{e}^{\sin ^{2} w} \cos w d w\right. \\
& -4 \cos ^{4} \theta \int_{0}^{\theta} \mathrm{e}^{\sin ^{2} w} \cos ^{5} w d w-2 \int_{0}^{\theta} \mathrm{e}^{\sin ^{2} w} \cos ^{5} w d w+6 \cos ^{2} \theta \int_{0}^{\theta} \mathrm{e}^{\sin ^{2} w} \cos ^{5} w d w \\
& \left.+2 \cos ^{4} \theta \int_{0}^{\theta} \mathrm{e}^{\sin ^{2} w} \cos ^{3} w d w+\int_{0}^{\theta} \mathrm{e}^{\sin ^{2} w} \cos ^{3} w d w-3 \cos ^{2} \theta \int_{0}^{\theta} \mathrm{e}^{\sin ^{2} w} \cos ^{3} w d w\right), \\
s_{0,2}(\theta)= & -4 \cos ^{6} \theta \int_{0}^{\theta} \mathrm{e}^{\sin ^{2} w} \cos ^{5} w d w+2 \int_{0}^{\theta} \mathrm{e}^{\sin ^{2} w} \cos ^{5} w d w-2 \cos ^{4} \theta \int_{0}^{\theta} \mathrm{e}^{\sin ^{2} w} \cos ^{5} w d w \\
& +2 \cos ^{6} \theta \int_{0}^{\theta} \mathrm{e}^{\sin ^{2} w} \cos ^{3} w d w-\int_{0}^{\theta} \mathrm{e}^{\sin ^{2} w} \cos ^{3} w d w+\cos ^{4} \theta \int_{0}^{\theta} \mathrm{e}^{2} w \cos ^{3} w d w, \\
s_{0,3}(\theta)= & \cos ^{2} \theta\left(2 \int_{0}^{\theta} \mathrm{e}^{\sin ^{2} w} \cos ^{5} w d w+4 \cos ^{2} \theta \int_{0}^{\theta} \mathrm{e}^{\sin ^{2} w} \cos ^{5} w d w-8 \cos ^{4} \theta \int_{0}^{\theta} \mathrm{e}^{\sin ^{2} w} \cos ^{5} w d w\right. \\
& \left.-\int_{0}^{\theta} \mathrm{e}^{\sin ^{2} w} \cos ^{3} w d w-2 \cos ^{2} \theta \int_{0}^{\theta} \mathrm{e}^{\sin ^{2} w} \cos ^{3} w d w+4 \cos ^{4} \theta \int_{0}^{\theta} \mathrm{e}^{\sin ^{2} w} \cos ^{3} w d w\right),
\end{aligned}
$$




$$
\begin{aligned}
& s_{0,4}(\theta)=\cos ^{3} \theta \sin \theta\left(2 \cos ^{2} \theta \int_{0}^{\theta} \mathrm{e}^{\sin ^{2} w} \cos w d w-\int_{0}^{\theta} \mathrm{e}^{\sin ^{2} w} \cos w d w-4 \cos ^{2} \theta \int_{0}^{\theta} \mathrm{e}^{\sin ^{2} w} \cos ^{5} w d w\right. \\
& \left.+2 \int_{0}^{\theta} \mathrm{e}^{\sin ^{2} w} \cos ^{5} w d w+2 \cos ^{2} \theta \int_{0}^{\theta} \mathrm{e}^{\sin ^{2} w} \cos ^{3} w d w-\int_{0}^{\theta} \mathrm{e}^{\sin ^{2} w} \cos ^{3} w d w\right), \\
& s_{0,5}(\theta)=\cos ^{3} \theta \sin \theta\left(2 \cos ^{2} \theta \int_{0}^{\theta} \mathrm{e}^{\sin ^{2} w} \sin ^{3} w d w-\int_{0}^{\theta} \mathrm{e}^{\sin ^{2} w} \sin ^{3} w d w\right. \\
& \left.+4 \cos ^{2} \theta \int_{0}^{\theta} \mathrm{e}^{\sin ^{2} w} \sin ^{3} w \cos ^{2} w d w-2 \int_{0}^{\theta} \mathrm{e}^{\sin ^{2} w} \sin ^{3} w \cos ^{2} w d w\right), \\
& s_{0,6}(\theta)=\cos ^{3} \theta \sin \theta\left(2 \cos ^{2} \theta \int_{0}^{\theta} \mathrm{e}^{\sin ^{2} w} \cos ^{2} w \sin w d w-\int_{0}^{\theta} \mathrm{e}^{\sin ^{2} w} \cos ^{2} w \sin w d w\right. \\
& \left.+4 \cos ^{2} \theta \int_{0}^{\theta} \mathrm{e}^{\sin ^{2} w} \cos ^{4} w \sin w d w-2 \int_{0}^{\theta} \mathrm{e}^{\sin ^{2} w} \cos ^{4} w \sin w d w\right), \\
& s_{0,7}(\theta)=\cos ^{3} \theta \sin \theta\left(\int_{0}^{\theta} \mathrm{e}^{\sin ^{2} w} \sin ^{3} w d w+2 \cos ^{2} \theta \int_{0}^{\theta} \mathrm{e}^{\sin ^{2} w} \sin ^{3} w d w\right. \\
& \left.+2 \int_{0}^{\theta} \mathrm{e}^{\sin ^{2} w} \sin ^{3} w \cos ^{2} w d w+4 \cos ^{2} \theta \int_{0}^{\theta} \mathrm{e}^{\sin ^{2} w} \sin ^{3} w \cos ^{2} w d w\right), \\
& s_{0,8}(\theta)=-\cos ^{2} \theta\left(6 \cos ^{4} \theta \int_{0}^{\theta} \mathrm{e}^{\sin ^{2} w} \cos ^{3} w d w+3 \int_{0}^{\theta} \mathrm{e}^{\sin ^{2} w} \cos ^{3} w d w-9 \cos ^{2} \theta \int_{0}^{\theta} \mathrm{e}^{\sin ^{2} w} \cos ^{3} w d w\right. \\
& -4 \cos ^{4} \theta \int_{0}^{\theta} \mathrm{e}^{\sin ^{2} w} \cos ^{5} w d w-2 \int_{0}^{\theta} \mathrm{e}^{\sin ^{2} w} \cos ^{5} w d w+6 \cos ^{2} \theta \int_{0}^{\theta} \mathrm{e}^{\sin ^{2} w} \cos ^{5} w d w \\
& \left.-2 \cos ^{4} \theta \int_{0}^{\theta} \mathrm{e}^{\sin ^{2} w} \cos w d w-\int_{0}^{\theta} \mathrm{e}^{\sin ^{2} w} \cos w d w+3 \cos ^{2} \theta \int_{0}^{\theta} \mathrm{e}^{\sin ^{2} w} \cos w d w\right), \\
& s_{0,9}(\theta)=-6 \cos ^{6} \theta \int_{0}^{\theta} \mathrm{e}^{\sin ^{2} w} \cos ^{3} w d w+3 \int_{0}^{\theta} \mathrm{e}^{\sin ^{2} w} \cos ^{3} w d w-3 \cos ^{4} \theta \int_{0}^{\theta} \mathrm{e}^{\sin ^{2} w} \cos ^{3} w d w \\
& +4 \cos ^{6} \theta \int_{0}^{\theta} \mathrm{e}^{\sin ^{2} w} \cos ^{5} w d w-2 \int_{0}^{\theta} \mathrm{e}^{\sin ^{2} w} \cos ^{5} w d w+2 \cos ^{4} \theta \int_{0}^{\theta} \mathrm{e}^{\sin ^{2} w} \cos ^{5} w d w \\
& +2 \cos ^{6} \theta \int_{0}^{\theta} \mathrm{e}^{\sin ^{2} w} \cos w d w-\int_{0}^{\theta} \mathrm{e}^{\sin ^{2} w} \cos w d w+\cos ^{4} \theta \int_{0}^{\theta} \mathrm{e}^{\sin ^{2} w} \cos w d w, \\
& s_{0,10}(\theta)=-\cos ^{2} \theta\left(-3 \int_{0}^{\theta} \mathrm{e}^{\sin ^{2} w} \cos ^{3} w d w-6 \cos ^{2} \theta \int_{0}^{\theta} \mathrm{e}^{\sin ^{2} w} \cos ^{3} w d w+12 \cos ^{4} \theta \int_{0}^{\theta} \mathrm{e}^{\sin ^{2} w} \cos ^{3} w d w\right. \\
& +2 \int_{0}^{\theta} \mathrm{e}^{\sin ^{2} w} \cos ^{5} w d w+4 \cos ^{2} \theta \int_{0}^{\theta} \mathrm{e}^{\sin ^{2} w} \cos ^{5} w d w-8 \cos ^{4} \theta \int_{0}^{\theta} \mathrm{e}^{\sin ^{2} w} \cos ^{5} w d w \\
& \left.+\int_{0}^{\theta} \mathrm{e}^{\sin ^{2} w} \cos w d w+2 \cos ^{2} \theta \int_{0}^{\theta} \mathrm{e}^{\sin ^{2} w} \cos w d w-4 \cos ^{4} \theta \int_{0}^{\theta} \mathrm{e}^{\sin ^{2} w} \cos w d w\right) \\
& s_{0,11}(\theta)=\cos \theta \sin \theta\left(2 \cos ^{4} \theta \int_{0}^{\theta} \mathrm{e}^{\sin ^{2} w} \cos ^{2} w \sin w d w+\int_{0}^{\theta} \mathrm{e}^{\sin ^{2} w} \cos ^{2} w \sin w d w\right. \\
& -3 \cos ^{2} \theta \int_{0}^{\theta} \mathrm{e}^{\sin ^{2} w} \cos ^{2} w \sin w d w-4 \cos ^{4} \theta \int_{0}^{\theta} \mathrm{e}^{\sin ^{2} w} \cos ^{4} w \sin w d w \\
& \left.-2 \int_{0}^{\theta} \mathrm{e}^{\sin ^{2} w} \cos ^{4} w \sin w d w+6 \cos ^{2} \theta \int_{0}^{\theta} \mathrm{e}^{\sin ^{2} w} \cos ^{4} w \sin w d w\right),
\end{aligned}
$$




$$
\begin{aligned}
& s_{0,12}(\theta)=-\cos \theta \sin \theta\left(2 \cos ^{4} \theta \int_{0}^{\theta} \mathrm{e}^{\sin ^{2} w} \sin ^{3} w d w+\int_{0}^{\theta} \mathrm{e}^{\sin ^{2} w} \sin ^{3} w d w\right. \\
& -3 \cos ^{2} \theta \int_{0}^{\theta} \mathrm{e}^{\sin w^{2}} \sin ^{3} w d w+4 \cos ^{4} \theta \int_{0}^{\theta} \mathrm{e}^{\sin ^{2} w} \sin ^{3} w \cos ^{2} w d w \\
& \left.+2 \int_{0}^{\theta} \mathrm{e}^{\sin ^{2} w} \sin ^{3} w \cos ^{2} w d w-6 \cos ^{2} \theta \int_{0}^{\theta} \mathrm{e}^{\sin ^{2} w} \sin ^{3} w \cos ^{2} w d w\right), \\
& s_{0,13}(\theta)=-\cos \theta \sin \theta\left(2 \cos ^{4} \theta \int_{0}^{\theta} \mathrm{e}^{\sin ^{2} w} \cos ^{2} w \sin w d w+\int_{0}^{\theta} \mathrm{e}^{\sin ^{2} w} \cos ^{2} w \sin w d w\right. \\
& -3 \cos ^{2} \theta \int_{0}^{\theta} \mathrm{e}^{\sin ^{2} w} \cos ^{2} w \sin w d w+4 \cos ^{4} \theta \int_{0}^{\theta} \mathrm{e}^{\sin ^{2} w} \cos ^{4} w \sin w d w \\
& \left.+2 \int_{0}^{\theta} \mathrm{e}^{\sin ^{2} w} \cos ^{4} w \sin w d w-6 \cos ^{2} \theta \int_{0}^{\theta} \mathrm{e}^{\sin ^{2} w} \cos ^{4} w \sin w d w\right), \\
& s_{0,14}(\theta)=-\cos ^{3} \theta \sin \theta\left(2 \cos ^{2} \theta \int_{0}^{\theta} \mathrm{e}^{\sin w^{2}} \cos ^{2} w \sin w d w-\int_{0}^{\theta} \mathrm{e}^{\sin ^{2} w} \cos ^{2} w \sin w d w\right. \\
& \left.-4 \cos ^{2} \theta \int_{0}^{\theta} \mathrm{e}^{\sin ^{2} w} \cos ^{4} w \sin w d w+2 \int_{0}^{\theta} \mathrm{e}^{\sin ^{2} w} \cos ^{4} w \sin w d w\right), \\
& s_{0,15}(\theta)=\cos \theta \sin \theta\left(-\cos ^{2} \theta \int_{0}^{\theta} \mathrm{e}^{\sin ^{2} w} \cos ^{2} w \sin w d w-\int_{0}^{\theta} \mathrm{e}^{\sin ^{2} w} \cos ^{2} w \sin w d w\right. \\
& +2 \cos ^{4} \theta \int_{0}^{\theta} \mathrm{e}^{\sin ^{2} w} \cos ^{2} w \sin w d w+2 \cos ^{2} \theta \int_{0}^{\theta} \mathrm{e}^{\sin ^{2} w} \cos ^{4} w \sin w d w \\
& \left.+2 \int_{0}^{\theta} \mathrm{e}^{\sin ^{2} w} \cos ^{4} w \sin w d w-4 \cos ^{4} \theta \int_{0}^{\theta} \mathrm{e}^{\sin ^{2} w} \cos ^{4} w \sin w d w\right), \\
& s_{0,16}(\theta)=-\cos ^{3} \theta \sin \theta\left(\int_{0}^{\theta} \mathrm{e}^{\sin ^{2} w} \cos ^{2} w \sin w d w+2 \cos ^{2} \theta \int_{0}^{\theta} \mathrm{e}^{\sin ^{2} w} \cos ^{2} w \sin w d w\right. \\
& \left.-2 \int_{0}^{\theta} \mathrm{e}^{\sin ^{2} w} \cos ^{4} w \sin w d w-4 \cos ^{2} \theta \int_{0}^{\theta} \mathrm{e}^{\sin ^{2} w} \cos ^{4} w \sin w d w\right), \\
& s_{0,17}(\theta)=\cos ^{2} \theta\left(-4 \cos ^{4} \theta \int_{0}^{\theta} \mathrm{e}^{\sin ^{2} w} \cos ^{5} w d w-2 \int_{0}^{\theta} \mathrm{e}^{\sin ^{2} w} \cos ^{5} w d w\right. \\
& +6 \cos ^{2} \theta \int_{0}^{\theta} \mathrm{e}^{\sin ^{2} w} \cos ^{5} w d w+2 \cos ^{4} \theta \int_{0}^{\theta} \mathrm{e}^{\sin ^{2} w} \cos ^{3} w d w+\int_{0}^{\theta} \mathrm{e}^{\sin ^{2} w} \cos ^{3} w d w \\
& \left.-3 \cos ^{2} \theta \int_{0}^{\theta} \mathrm{e}^{\sin ^{2} w} \cos ^{3} w d w\right) \\
& s_{0,18}(\theta)=\cos ^{2} \theta\left(2 \cos ^{4} \theta \int_{0}^{\theta} \mathrm{e}^{\sin ^{2} w} \cos ^{2} w \sin w d w,\right. \\
& +\int_{0}^{\theta} \mathrm{e}^{\sin ^{2} w} \cos ^{2} w \sin w d w-3 \cos ^{2} \theta \int_{0}^{\theta} \mathrm{e}^{\sin ^{2} w} \cos ^{2} w \sin w d w \\
& -4 \cos ^{4} \theta \int_{0}^{\theta} \mathrm{e}^{\sin ^{2} w} \cos ^{4} w \sin w d w-2 \int_{0}^{\theta} \mathrm{e}^{\sin ^{2} w} \cos ^{4} w \sin w d w \\
& \left.+6 \cos ^{2} \theta \int_{0}^{\theta} \mathrm{e}^{\sin ^{2} w} \cos ^{4} w \sin w d w\right),
\end{aligned}
$$




$$
\begin{aligned}
& s_{0,19}(\theta)=\cos \theta \sin \theta\left(-4 \cos ^{4} \theta \int_{0}^{\theta} \mathrm{e}^{\sin ^{2} w} \cos ^{5} w d w-2 \int_{0}^{\theta} \mathrm{e}^{\sin ^{2} w} \cos ^{5} w d w\right. \\
& +6 \cos ^{2} \theta \int_{0}^{\theta} \mathrm{e}^{\sin w^{2}} \cos ^{5} w d w+2 \cos ^{4} \theta \int_{0}^{\theta} \mathrm{e}^{\sin ^{2} w} \cos ^{3} w d w+\int_{0}^{\theta} \mathrm{e}^{\sin ^{2} w} \cos ^{3} w d w \\
& \left.-3 \cos ^{2} \theta \int_{0}^{\theta} \mathrm{e}^{\sin ^{2} w} \cos ^{3} w d w\right) \\
& s_{0,20}(\theta)=\cos ^{3} \theta \sin \theta\left(6 \cos ^{2} \theta \int_{0}^{\theta} \mathrm{e}^{\sin w^{2}} \cos ^{3} w d w\right. \\
& -3 \int_{0}^{\theta} \mathrm{e}^{\sin ^{2} w} \cos ^{3} w d w-4 \cos ^{2} \theta \int_{0}^{\theta} \mathrm{e}^{\sin ^{2} w} \cos ^{5} w d w \\
& \left.+2 \int_{0}^{\theta} \mathrm{e}^{\sin ^{2} w} \cos ^{5} w d w-2 \cos ^{2} \theta \int_{0}^{\theta} \mathrm{e}^{\sin ^{2} w} \cos w d w+\int_{0}^{\theta} \mathrm{e}^{\sin ^{2} w} \cos w d w\right), \\
& s_{0,21}(\theta)=\cos ^{2} \theta\left(-\int_{0}^{\theta} \mathrm{e}^{\sin ^{2} w} \cos ^{2} w \sin w d w-2 \cos ^{2} \theta \int_{0}^{\theta} \mathrm{e}^{\sin ^{2} w} \cos ^{2} w \sin w d w\right. \\
& +4 \cos ^{4} \theta \int_{0}^{\theta} \mathrm{e}^{\sin ^{2} w} \cos ^{2} w \sin w d w+2 \int_{0}^{\theta} \mathrm{e}^{\sin ^{2} w} \cos ^{4} w \sin w d w \\
& \left.+4 \cos ^{2} \theta \int_{0}^{\theta} \mathrm{e}^{\sin ^{2} w} \cos ^{4} w \sin w d w-8 \cos ^{4} \theta \int_{0}^{\theta} \mathrm{e}^{\sin ^{2} w} \cos ^{4} w \sin w d w\right), \\
& s_{0,22}(\theta)=\cos ^{3} \theta \sin \theta\left(\int_{0}^{\theta} \mathrm{e}^{\sin ^{2} w} \cos ^{2} w \sin w d w+2 \cos ^{2} \theta \int_{0}^{\theta} \mathrm{e}^{\sin ^{2} w} \cos ^{2} w \sin w d w\right. \\
& \left.+2 \int_{0}^{\theta} \mathrm{e}^{\sin ^{2} w} \cos ^{4} w \sin w d w+4 \cos ^{2} \theta \int_{0}^{\theta} \mathrm{e}^{\sin ^{2} w} \cos ^{4} w \sin w d w\right), \\
& s_{0,23}(\theta)=-2 \cos ^{6} \theta \int_{0}^{\theta} \mathrm{e}^{\sin ^{2} w} \cos w d w+\int_{0}^{\theta} \mathrm{e}^{\sin ^{2} w} \cos w d w-\cos ^{4} \theta \int_{0}^{\theta} \mathrm{e}^{\sin ^{2} w} \cos w d w \\
& +4 \cos ^{6} \theta \int_{0}^{\theta} \mathrm{e}^{\sin ^{2} w} \cos ^{5} w d w-2 \int_{0}^{\theta} \mathrm{e}^{\sin ^{2} w} \cos ^{5} w d w+2 \cos ^{4} \theta \int_{0}^{\theta} \mathrm{e}^{\sin ^{2} w} \cos ^{5} w d w \\
& -2 \cos ^{6} \theta \int_{0}^{\theta} \mathrm{e}^{\sin ^{2} w} \cos ^{3} w d w+\int_{0}^{\theta} \mathrm{e}^{\sin ^{2} w} \cos ^{3} w d w-\cos \theta^{4} \int_{0}^{\theta} \mathrm{e}^{\sin ^{2} w} \cos ^{3} w d w, \\
& s_{0,24(\theta)}=-2\left(\int_{0}^{\theta} \mathrm{e}^{\sin ^{2} w} \sin ^{3} w d w\right) \cos ^{6} \theta+\int_{0}^{\theta} \mathrm{e}^{\sin ^{2} w} \sin ^{3} w d w-\cos \theta^{4} \int_{0}^{\theta} \mathrm{e}^{\sin ^{2} w} \sin ^{3} w d w \\
& -4\left(\int_{0}^{\theta} \mathrm{e}^{\sin ^{2} w} \sin ^{3} w \cos ^{2} w d w\right) \cos ^{6} \theta+2 \int_{0}^{\theta} \mathrm{e}^{\sin w^{2}} \sin ^{3} w \cos ^{2} w d w \\
& -2 \cos ^{4} \theta \int_{0}^{\theta} \mathrm{e}^{\sin ^{2} w} \sin ^{3} w \cos ^{2} w d w \\
& s_{0,25}(\theta)=-\cos ^{2} \theta\left(-\int_{0}^{\theta} \mathrm{e}^{\sin ^{2} w} \cos w d w-2 \cos \theta^{2} \int_{0}^{\theta} \mathrm{e}^{\sin w^{2}} \cos w d w+4 \cos ^{4} \theta \int_{0}^{\theta} \mathrm{e}^{\sin ^{2} w} \cos w d w\right. \\
& +2 \int_{0}^{\theta} \mathrm{e}^{\sin ^{2} w} \cos ^{5} w d w+4 \cos ^{2} \theta \int_{0}^{\theta} \mathrm{e}^{\sin ^{2} w} \cos ^{5} w d w-8 \cos ^{4} \theta \int_{0}^{\theta} \mathrm{e}^{\sin ^{2} w} \cos ^{5} w d w \\
& \left.-\int_{0}^{\theta} \mathrm{e}^{\sin ^{2} w} \cos ^{3} w d w-2 \cos ^{2} \theta \int_{0}^{\theta} \mathrm{e}^{\sin ^{2} w} \cos ^{3} w d w+4 \cos ^{4} \theta \int_{0}^{\theta} \mathrm{e}^{\sin ^{2} w} \cos ^{3} w d w\right)
\end{aligned}
$$




$$
\begin{aligned}
& s_{0,26}(\theta)=-\cos \theta \sin \theta\left(-\cos ^{2} \theta \int_{0}^{\theta} \mathrm{e}^{\sin ^{2} w} \cos ^{2} w \sin w d w-\int_{0}^{\theta} \mathrm{e}^{\sin ^{2} w} \cos ^{2} w \sin w d w\right. \\
& +2 \cos ^{4} \theta \int_{0}^{\theta} \mathrm{e}^{\sin ^{2} w} \cos ^{2} w \sin w d w-2 \cos ^{2} \theta \int_{0}^{\theta} \mathrm{e}^{\sin ^{2} w} \cos ^{4} w \sin w d w-2 \int_{0}^{\theta} \mathrm{e}^{\sin ^{2} w} \cos ^{4} w \sin w d w \\
& \left.+4 \cos ^{4} \theta \int_{0}^{\theta} \mathrm{e}^{\sin ^{2} w} \cos ^{4} w \sin w d w\right) \\
& s_{0,27}(\theta)=-\cos \theta \sin \theta\left(-\cos ^{2} \theta \int_{0}^{\theta} \mathrm{e}^{\sin ^{2} w} \sin ^{3} w d w\right)-\int_{0}^{\theta} \mathrm{e}^{\sin ^{2} w} \sin ^{3} w d w \\
& +2 \cos ^{4} \theta \int_{0}^{\theta} \mathrm{e}^{\sin w^{2}} \sin ^{3} w d w-2 \cos ^{2} \theta \int_{0}^{\theta} \mathrm{e}^{\sin ^{2} w} \sin ^{3} w \cos ^{2} w d w-2 \int_{0}^{\theta} \mathrm{e}^{\sin ^{2} w} \sin ^{3} w \cos ^{2} w d w \\
& \left.+4 \cos ^{4} \theta \int_{0}^{\theta} \mathrm{e}^{\sin ^{2} w} \sin ^{3} w \cos ^{2} w d w\right) \\
& s_{0,28}(\theta)=-\cos \theta \sin \theta\left(-3 \cos ^{2} \theta \int_{0}^{\theta} \mathrm{e}^{\sin ^{2} w} \cos ^{3} w d w-3 \int_{0}^{\theta} \mathrm{e}^{\sin ^{2} w} \cos ^{3} w d w\right. \\
& +6 \cos ^{4} \theta \int_{0}^{\theta} \mathrm{e}^{\sin ^{2} w} \cos ^{3} w d w+2 \cos ^{2} \theta \int_{0}^{\theta} \mathrm{e}^{\sin ^{2} w} \cos ^{5} w d w \\
& +2 \int_{0}^{\theta} \mathrm{e}^{\sin ^{2} w} \cos ^{5} w d w-4 \cos ^{4} \theta \int_{0}^{\theta} \mathrm{e}^{\sin ^{2} w} \cos ^{5} w d w+\cos ^{2} \theta \int_{0}^{\theta} \mathrm{e}^{\sin ^{2} w} \cos w d w \\
& \left.+\int_{0}^{\theta} \mathrm{e}^{\sin ^{2} w} \cos w d w-2 \cos ^{4} \theta \int_{0}^{\theta} \mathrm{e}^{\sin ^{2} w} \cos w d w\right) \\
& s_{0,29}(\theta)=\cos ^{3} \theta \sin \theta\left(3 \int_{0}^{\theta} \mathrm{e}^{\sin ^{2} w} \cos ^{3} w d w+6 \cos ^{2} \theta \int_{0}^{\theta} \mathrm{e}^{\sin ^{2} w} \cos ^{3} w d w-2 \int_{0}^{\theta} \mathrm{e}^{\sin ^{2} w} \cos ^{5} w d w\right. \\
& \left.-4 \cos ^{2} \theta \int_{0}^{\theta} \mathrm{e}^{\sin ^{2} w} \cos ^{5} w d w-\int_{0}^{\theta} \mathrm{e}^{\sin w^{2}} \cos w d w-2 \cos ^{2} \theta \int_{0}^{\theta} \mathrm{e}^{\sin ^{2} w} \cos w d w\right), \\
& s_{0,30}(\theta)=-\cos \theta \sin \theta\left(6 \cos ^{4} \theta \int_{0}^{\theta} \mathrm{e}^{\sin ^{2} w} \cos ^{3} w d w+3 \int_{0}^{\theta} \mathrm{e}^{\sin ^{2} w} \cos ^{3} w d w\right. \\
& -9 \cos ^{2} \theta \int_{0}^{\theta} \mathrm{e}^{\sin w^{2}} \cos ^{3} w d w-4 \cos ^{4} \theta \int_{0}^{\theta} \mathrm{e}^{\sin ^{2} w} \cos ^{5} w d w-2 \int_{0}^{\theta} \mathrm{e}^{\sin ^{2} w} \cos ^{5} w d w \\
& +6 \cos ^{2} \theta \int_{0}^{\theta} \mathrm{e}^{\sin ^{2} w} \cos ^{5} w d w-2 \cos ^{4} \theta \int_{0}^{\theta} \mathrm{e}^{\sin ^{2} w} \cos w d w-\int_{0}^{\theta} \mathrm{e}^{\sin ^{2} w} \cos w d w \\
& \left.+3 \cos ^{2} \theta \int_{0}^{\theta} \mathrm{e}^{\sin ^{2} w} \cos w d w\right), \\
& s_{0,31}(\theta)=-\cos ^{3} \theta \sin \theta\left(-4 \cos ^{2} \theta \int_{0}^{\theta} \mathrm{e}^{\sin w^{2}} \cos ^{5} w d w+2 \int_{0}^{\theta} \mathrm{e}^{\sin ^{2} w} \cos ^{5} w d w\right. \\
& \left.+2 \cos ^{2} \theta \int_{0}^{\theta} \mathrm{e}^{\sin ^{2} w} \cos ^{3} w d w-\int_{0}^{\theta} \mathrm{e}^{\sin ^{2} w} \cos ^{3} w d w\right), \\
& s_{0,32}(\theta)=\cos ^{3} \theta \sin \theta\left(\int_{0}^{\theta} \mathrm{e}^{\sin ^{2} w} \cos w d w+2 \cos ^{2} \theta \int_{0}^{\theta} \mathrm{e}^{\sin ^{2} w} \cos w d w-2 \int_{0}^{\theta} \mathrm{e}^{\sin w^{2}} \cos ^{5} w d w\right. \\
& \left.-4 \cos ^{2} \theta \int_{0}^{\theta} \mathrm{e}^{\sin ^{2} w} \cos ^{5} w d w+\int_{0}^{\theta} \mathrm{e}^{\sin ^{2} w} \cos ^{3} w d w+2 \cos ^{2} \theta \int_{0}^{\theta} \mathrm{e}^{\sin ^{2} w} \cos ^{3} w d w\right),
\end{aligned}
$$




$$
\begin{aligned}
& s_{0,33}(\theta)=-\cos ^{2} \theta\left(-\int_{0}^{\theta} \mathrm{e}^{\sin ^{2} w} \cos ^{2} w \sin w d w-2 \cos ^{2} \theta \int_{0}^{\theta} \mathrm{e}^{\sin ^{2} w} \cos ^{2} w \sin w d w\right. \\
& +4 \cos ^{4} \theta \int_{0}^{\theta} \mathrm{e}^{\sin ^{2} w} \cos ^{2} w \sin w d w-2 \int_{0}^{\theta} \mathrm{e}^{\sin ^{2} w} \cos ^{4} w \sin w d w \\
& \left.-4 \cos ^{2} \theta \int_{0}^{\theta} \mathrm{e}^{\sin ^{2} w} \cos ^{4} w \sin w d w+8 \cos ^{4} \theta \int_{0}^{\theta} \mathrm{e}^{\sin ^{2} w} \cos ^{4} w \sin w d w\right), \\
& s_{0,34}(\theta)=-\cos ^{2} \theta\left(-\int_{0}^{\theta} \mathrm{e}^{\sin ^{2} w} \sin ^{3} w d w-2 \cos ^{2} \theta \int_{0}^{\theta} \mathrm{e}^{\sin ^{2} w} \sin ^{3} w d w\right. \\
& +4 \cos ^{4} \theta \int_{0}^{\theta} \mathrm{e}^{\sin ^{2} w} \sin ^{3} w d w-2 \int_{0}^{\theta} \mathrm{e}^{\sin ^{2} w} \sin ^{3} w \cos ^{2} w d w \\
& \left.-4 \cos ^{2} \theta \int_{0}^{\theta} \mathrm{e}^{\sin ^{2} w} \sin ^{3} w \cos ^{2} w d w+8 \cos ^{4} \theta \int_{0}^{\theta} \mathrm{e}^{\sin ^{2} w} \sin ^{3} w \cos ^{2} w d w\right), \\
& s_{0,35}(\theta)=-2 \cos ^{6} \theta \int_{0}^{\theta} \mathrm{e}^{\sin ^{2} w} \cos ^{2} w \sin w d w+\int_{0}^{\theta} \mathrm{e}^{\sin ^{2} w} \cos ^{2} w \sin w d w \\
& -\cos ^{4} \theta \int_{0}^{\theta} \mathrm{e}^{\sin ^{2} w} \cos ^{2} w \sin w d w-4 \cos ^{6} \theta \int_{0}^{\theta} \mathrm{e}^{\sin ^{2} w} \cos ^{4} w \sin w d w \\
& +2 \int_{0}^{\theta} \mathrm{e}^{\sin ^{2} w} \cos ^{4} w \sin w d w-2 \cos ^{4} \theta \int_{0}^{\theta} \mathrm{e}^{\sin ^{2} w} \cos ^{4} w \sin w d w, \\
& s_{0,36}(\theta)=-\cos \theta \sin \theta\left(-\cos ^{2} \theta \int_{0}^{\theta} \mathrm{e}^{\sin ^{2} w} \cos w d w-\int_{0}^{\theta} \mathrm{e}^{\sin w^{2}} \cos w d w\right. \\
& +2 \cos ^{4} \theta \int_{0}^{\theta} \mathrm{e}^{\sin ^{2} w} \cos w d w+2 \cos ^{2} \theta \int_{0}^{\theta} \mathrm{e}^{\sin ^{2} w} \cos ^{5} w d w+2 \int_{0}^{\theta} \mathrm{e}^{\sin ^{2} w} \cos ^{5} w d w \\
& -4 \cos ^{4} \theta \int_{0}^{\theta} \mathrm{e}^{\sin ^{2} w} \cos ^{5} w d w-\cos ^{2} \theta \int_{0}^{\theta} \mathrm{e}^{\sin ^{2} w} \cos ^{3} w d w-\int_{0}^{\theta} \mathrm{e}^{\sin w^{2}} \cos ^{3} w d w \\
& \left.+2 \cos ^{4} \theta \int_{0}^{\theta} \mathrm{e}^{\sin ^{2} w} \cos ^{3} w d w\right) \\
& s_{0,37}(\theta)=-\cos ^{3} \theta \sin \theta\left(-2 \int_{0}^{\theta} \mathrm{e}^{\sin ^{2} w} \cos ^{5} w d w-4 \cos ^{2} \theta \int_{0}^{\theta} \mathrm{e}^{\sin ^{2} w} \cos ^{5} w d w\right. \\
& \left.+\int_{0}^{\theta} \mathrm{e}^{\sin ^{2} w} \cos ^{3} w d w+2 \cos ^{2} \theta \int_{0}^{\theta} \mathrm{e}^{\sin ^{2} w} \cos ^{3} w d w\right), \\
& s_{0,38}(\theta)=-\cos ^{2} \theta\left(2 \cos ^{4} \theta \int_{0}^{\theta} \mathrm{e}^{\sin ^{2} w} \cos ^{2} w \sin w d w+\int_{0}^{\theta} \mathrm{e}^{\sin ^{2} w} \cos ^{2} w \sin w d w\right. \\
& -3 \cos ^{2} \theta \int_{0}^{\theta} \mathrm{e}^{\sin ^{2} w} \cos ^{2} w \sin w d w+4 \cos ^{4} \theta \int_{0}^{\theta} \mathrm{e}^{\sin ^{2} w} \cos ^{4} w \sin w d w \\
& \left.+2 \int_{0}^{\theta} \mathrm{e}^{\sin ^{2} w} \cos ^{4} w \sin w d w-6 \cos ^{2} \theta \int_{0}^{\theta} \mathrm{e}^{\sin ^{2} w} \cos ^{4} w \sin w d w\right), \\
& s_{0,39}(\theta)=\cos \theta \sin \theta\left(2 \cos ^{2} \theta \int_{0}^{\theta} \mathrm{e}^{\sin ^{2} w} \cos ^{5} w d w+2 \int_{0}^{\theta} \mathrm{e}^{\sin ^{2} w} \cos ^{5} w d w-4 \cos ^{4} \theta \int_{0}^{\theta} \mathrm{e}^{\sin w^{2}} \cos ^{5} w d w\right. \\
& \left.-\cos ^{2} \theta \int_{0}^{\theta} \mathrm{e}^{\sin ^{2} w} \cos ^{3} w d w-\int_{0}^{\theta} \mathrm{e}^{\sin ^{2} w} \cos ^{3} w d w+2 \cos ^{4} \theta \int_{0}^{\theta} \mathrm{e}^{\sin ^{2} w} \cos ^{3} w d w\right),
\end{aligned}
$$




$$
\begin{aligned}
s_{0,40}(\theta)= & 2 \cos ^{6} \theta \int_{0}^{\theta} \mathrm{e}^{\sin ^{2} w} \cos ^{2} w \sin w d w-\int_{0}^{\theta} \mathrm{e}^{\sin ^{2} w} \cos ^{2} w \sin w d w+\cos ^{4} \theta \int_{0}^{\theta} \mathrm{e}^{\sin ^{2} w} \cos ^{2} w \sin w d w \\
& -4 \cos ^{6} \theta \int_{0}^{\theta} \mathrm{e}^{\sin ^{2} w} \cos ^{4} w \sin w d w+2 \int_{0}^{\theta} \mathrm{e}^{\sin ^{2} w} \cos ^{4} w \sin w d w-2 \cos ^{4} \theta \int_{0}^{\theta} \mathrm{e}^{\sin ^{2} w} \cos ^{4} w \sin w d w, \\
s_{0,41}(\theta)= & -\cos ^{2} \theta\left(2 \cos ^{4} \theta \int_{0}^{\theta} \mathrm{e}^{\sin ^{2} w} \sin ^{3} w d w+\int_{0}^{\theta} \mathrm{e}^{\sin ^{2} w} \sin ^{3} w d w-3 \cos ^{2} \theta \int_{0}^{\theta} \mathrm{e}^{\sin ^{2} w} \sin ^{3} w d w\right. \\
& +\left(4 \cos ^{4} \theta+2-6 \cos ^{2} \theta\right) \int_{0}^{\theta} \mathrm{e}^{\sin ^{2} w} \sin ^{3} w \cos ^{2} w d w, \\
s_{0,42}(\theta)= & -\cos \theta \sin \theta\left(2 \cos ^{4} \theta \int_{0}^{\theta} \mathrm{e}^{\sin ^{2} w} \cos w d w+\int_{0}^{\theta} \mathrm{e}^{\sin w^{2}} \cos w d w-3 \cos ^{2} \theta \int_{0}^{\theta} \mathrm{e}^{\sin ^{2} w} \cos w d w\right. \\
& -4 \cos ^{4} \theta \int_{0}^{\theta} \mathrm{e}^{\sin ^{2} w} \cos ^{5} w d w-2 \int_{0}^{\theta} \mathrm{e}^{\sin ^{2} w} \cos ^{5} w d w+6 \cos ^{2} \theta \int_{0}^{\theta} \mathrm{e}^{\sin ^{2} w} \cos ^{5} w d w \\
& \left.+2 \cos ^{4} \theta \int_{0}^{\theta} \mathrm{e}^{\sin ^{2} w} \cos ^{3} w d w+\int_{0}^{\theta} \mathrm{e}^{\sin ^{2} w} \cos ^{3} w d w-3 \cos ^{2} \theta \int_{0}^{\theta} \mathrm{e}^{\sin ^{2} w} \cos ^{3} w d w\right)
\end{aligned}
$$

Now we have

$$
\begin{aligned}
\tilde{s}_{1}(\theta)= & \tilde{s}_{1,1}(\theta) c_{03} c_{12}+\tilde{s}_{1,2}(\theta) c_{03} c_{30}+\tilde{s}_{1,3}(\theta) a_{03} c_{03}+\tilde{s}_{1,4}(\theta) a_{21} c_{03}+\tilde{s}_{1,5}(\theta) c_{30} c_{12}+\tilde{s}_{1,6}(\theta) c_{12} a_{21} \\
& +\tilde{s}_{1,7}(\theta) c_{30} a_{21}+\tilde{s}_{1,8}(\theta) a_{03} a_{21}+\tilde{s}_{1,9}(\theta) a_{03} c_{30}+\tilde{s}_{1,10}(\theta) a_{03} c_{12}+\tilde{s}_{1,11}(\theta) a_{12} c_{03}+\tilde{s}_{1,12}(\theta) a_{12} c_{21} \\
& +\tilde{s}_{1,13}(\theta) c_{03} c_{21}+\tilde{s}_{1,14}(\theta) a_{21} c_{21}+\tilde{s}_{1,15}(\theta) a_{21} a_{12}+\tilde{s}_{1,16}(\theta) c_{12} a_{12}+\tilde{s}_{1,17}(\theta) a_{03} c_{21} \\
& +\tilde{s}_{1,18}(\theta) a_{03} a_{12}+\tilde{s}_{1,19}(\theta) c_{30} c_{21}+\tilde{s}_{1,20}(\theta) c_{30} a_{12}+\tilde{s}_{1,21}(\theta) c_{12} c_{21}+\tilde{s}_{1,22}(\theta) a_{12}^{2} \\
& +\tilde{s}_{1,23}(\theta) c_{21}^{2}+\tilde{s}_{1,24}(\theta) c_{30}^{2}+\tilde{s}_{1,25}(\theta) a_{03}^{2}+\tilde{s}_{1,26}(\theta) c_{03}^{2}+\tilde{s}_{1,27}(\theta) a_{21}^{2}+\tilde{s}_{1,28}(\theta) c_{12}^{2},
\end{aligned}
$$

and $\tilde{s}_{1, i}(\theta)$ for $i=1 \cdots, 28$ satisfying the following expressions:

$$
\begin{aligned}
& \tilde{s}_{1,1}(\theta)=-\frac{1}{12}\left(16 \cos ^{8} \theta+34 \cos ^{6} \theta+37 \cos ^{4} \theta+8 \cos ^{2} \theta-5\right)\left(\cos ^{2} \theta-1\right)^{2}, \\
& \tilde{s}_{1,2}(\theta)=\frac{1}{12}\left(\cos ^{2} \theta-1\right)\left(16 \cos ^{10} \theta+38 \cos ^{8} \theta+53 \cos ^{6} \theta+15 \cos ^{4} \theta-7 \cos ^{2} \theta-7\right), \\
& \tilde{s}_{1,3}(\theta)=-\frac{1}{12}\left(16 \cos ^{8} \theta+14 \cos ^{6} \theta+15 \cos ^{4} \theta-16 \cos ^{2} \theta+1\right)\left(\cos ^{2} \theta-1\right)^{2}, \\
& \tilde{s}_{1,4}(\theta)=\frac{1}{12}\left(\cos ^{2} \theta-1\right)\left(16 \cos ^{10} \theta+18 \cos ^{8} \theta+19 \cos ^{6} \theta-15 \cos ^{4} \theta-\cos ^{2} \theta-1\right), \\
& \tilde{s}_{1,5}(\theta)=\frac{1}{12} \cos \theta \sin ^{5} \theta\left(2 \cos ^{2} \theta+1\right)\left(8 \cos ^{4} \theta+12 \cos ^{2} \theta+7\right), \\
& \tilde{s}_{1,6}(\theta)=\frac{1}{12} \cos \theta \sin ^{5} \theta\left(16 \cos ^{6} \theta+12 \cos ^{4} \theta-2 \cos ^{2} \theta+1\right), \\
& \tilde{s}_{1,7}(\theta)=\frac{1}{6} \cos 3 \sin ^{3} \theta\left(8 \cos ^{6} \theta+8 \cos ^{4} \theta+5 \cos ^{2} \theta-3\right), \\
& \tilde{s}_{1,8}(\theta)=\frac{1}{12} \cos \theta \sin ^{5} \theta\left(-1+2 \cos ^{2} \theta\right)\left(8 \cos ^{4} \theta+1\right), \\
& \tilde{s}_{1,9}(\theta)=\frac{1}{12} \cos \theta \sin ^{5} \theta\left(16 \cos ^{6} \theta+12 \cos ^{4} \theta+6 \cos ^{2} \theta-7\right), \\
& \tilde{s}_{1,10}(\theta)=\frac{1}{6} \cos ^{2} \theta \sin ^{7} \theta\left(8 \cos ^{4} \theta+4 \cos ^{2} \theta-3\right), \\
& \tilde{s}_{1,11}(\theta)=-\frac{4}{3} \cos ^{3} \theta \sin ^{3} \theta\left(\cos ^{6} \theta+\cos ^{4} \theta+\cos ^{2} \theta-1\right), \\
& \tilde{s}_{1,12}(\theta)=-\frac{2}{3} \cos ^{5} \theta \sin ^{3} \theta\left(4 \cos ^{4} \theta-\cos ^{2} \theta-1\right), \\
& \tilde{s}_{1,13}(\theta)=\frac{2}{3} \cos ^{3} \theta \sin ^{2} \theta\left(-5 \cos ^{2} \theta-2+5 \cos ^{6} \theta+4 \cos ^{4} \theta+4 \cos ^{8} \theta\right),
\end{aligned}
$$




$$
\begin{aligned}
& \tilde{s}_{1,14}(\theta)=-\frac{1}{12} \sin ^{2} \theta \cos ^{2} \theta\left(32 \cos ^{8} \theta-4 \cos ^{6} \theta-6 \cos ^{4} \theta-3 \cos ^{2} \theta-1\right) \\
& \tilde{s}_{1,15}(\theta)=\frac{1}{12} \sin ^{4} \theta \cos ^{2} \theta\left(-1+2 \cos ^{2} \theta\right)\left(8 \cos ^{4} \theta+\cos ^{2} \theta+1\right) \\
& \tilde{s}_{1,16}(\theta)=\frac{1}{12} \sin ^{6} \theta \cos ^{2} \theta\left(16 \cos ^{4} \theta+10 \cos ^{2} \theta-5\right) \\
& \tilde{s}_{1,17}(\theta)=-\frac{1}{12} \sin ^{4} \theta \cos ^{2} \theta\left(32 \cos ^{6} \theta-12 \cos ^{4} \theta-6 \cos ^{2} \theta+1\right) \\
& \tilde{s}_{1,18}(\theta)=\frac{1}{12} \sin ^{6} \theta \cos ^{2} \theta\left(8 \cos ^{2} \theta-1\right)\left(-1+2 \cos ^{2} \theta\right) \\
& \tilde{s}_{1,19}(\theta)=-\frac{1}{12} \sin ^{2} \theta \cos ^{2} \theta\left(32 \cos ^{8} \theta+36 \cos ^{6} \theta+14 \cos ^{4} \theta-21 \cos ^{2} \theta-7\right), \\
& \tilde{s}_{1,20}(\theta)=\frac{1}{12} \sin ^{4} \theta \cos ^{2} \theta\left(16 \cos ^{6} \theta+14 \cos ^{4} \theta+7 \cos ^{2} \theta-7\right) \\
& \tilde{s}_{1,21}(\theta)=-\frac{1}{12} \sin ^{4} \theta \cos ^{2} \theta\left(32 \cos ^{6} \theta+28 \cos ^{4} \theta-10 \cos ^{2} \theta-5\right) \\
& \tilde{s}_{1,22}(\theta)=\frac{1}{3} \cos ^{5} \theta \sin ^{5} \theta\left(-1+2 \cos ^{2} \theta\right) \\
& \tilde{s}_{1,23}(\theta)=\frac{1}{3} \cos ^{5} \theta \sin ^{2} \theta\left(2 \cos ^{2} \theta+1\right)\left(4 \cos ^{4} \theta-1-2 \cos ^{2} \theta\right) \\
& \tilde{s}_{1,24}(\theta)=\frac{1}{12} \cos ^{3} \theta \sin ^{3} \theta\left(2 \cos ^{2} \theta+1\right)\left(4 \cos ^{4} \theta+7 \cos ^{2} \theta+7\right) \\
& \tilde{s}_{1,25}(\theta)=\frac{1}{12} \cos ^{2} \theta \sin ^{7} \theta\left(2 \cos ^{2} \theta+1\right)\left(2 \cos ^{2} \theta-1\right)\left(-1+2 \cos ^{2} \theta\right) \\
& \tilde{s}_{1,26}(\theta)=\frac{1}{3} \cos ^{2} \theta \sin \theta\left(\cos ^{4} \theta+2 \cos ^{2} \theta+3\right)\left(2 \cos ^{6} \theta-1+\cos ^{4} \theta\right) \\
& \tilde{s}_{1,27}(\theta)=\frac{1}{12} \cos ^{3} \theta \sin ^{3} \theta\left(-1+2 \cos ^{2} \theta\right)\left(4 \cos ^{4} \theta+\cos ^{2} \theta+1\right) \\
& \tilde{s}_{1,28}(\theta)=\frac{1}{12} \cos \theta \sin ^{7} \theta\left(4 \cos ^{2} \theta+5\right)\left(2 \cos ^{2} \theta+1\right)
\end{aligned}
$$

[1] V.I. Arnold, Loss of stability of self-oscillation close to resonance and versal deformations of equivariant vector fields, Funct. Anal. Appl. 11 (1977), 1-10.

[2] V.I. Arnold and Yu Il'yashenko, Dynamical systems I. Ordinary differential equations, in Encyclopaedia Math. Sci., 1, Springer, Berlin, 1988.

[3] I.S. Berezin and N.P. Zhidkov, Computing Methods, vol. II. Pergamon Press, Oxford (1964).

[4] T.R. Blows, L.M. Perko, Bifurcation of limit cycles from centers and separatrix cycles of planar analytic systems, SIAM Rev. 36 (1994), 341-376.

[5] A. Buică, j. Giné and L. Llibre, A second order analysis of the periodic solutions for nonlinear periodic differential systems with a smaller parameter, Physica D 241 (2012) 528-533.

[6] A. Buică and L. Llibre, Averaging methods for finding periodic orbits via Brouwer degree, Bull. Sci. Math. 128 (2004), 7-22.

[7] A. Cima and J. Llibre, Algebraic and topological classification of the homogeneous cubic systems in the plane, J. Math. Anal. Appl. 147 (1990), 420-448. 
[8] C. Chicone and M. Jacobs, Bifurcation of limit cycles from quadratic isochrones, J. Differential Equations 91 (1991), 268-326.

[9] C. Christopher and C. Li, Limit cycles of differential equations, Advanced Courses in Mathematics, CRM Barcelona, Birkhäuser Verlag, Basel, 2007.

[10] F. Dumortier, J. Llibre and J. C. Artés, Qualitative theory of planar polynomial systems, Springer, 2006.

[11] H. Giacomini, J. Llibre and M. Viano, On the nonexistence, existence and uniqueness of limit cycles, Nonlinearity 9 (1996), 501-516.

[12] H. Giacomini, J. Llibre and M. Viano, On the shape of limit cycles that bifurcate from Hamiltonian centers, Nonlinear Anal. 41 (2000), 523-537.

[13] H. Giacomini, J. Llibre and M. Viano, On the shape of limit cycles that bifurcate from non-Hamiltonian centers, Nonlinear Anal. 43 (2001), 837-859.

[14] J. Giné, M. Grau and J. Llibre, Averaging theory at any order for computing periodic orbits, Physica D 250 (2013), 58-65.

[15] J. Guckenheimer and P. Holmes, Nonlinear Oscillations, dynamical systems and bifurcations of vector fields, Applied Mathematical Sciences, 42, Springer-Verlag, New York, 1986.

[16] D. Hilbert, Mthematische Probleme, Lecture at the Second International Congress of Mathematicians, Paris 1900; reprinted in Mathematical Developments Arising from Hilbert Problems (ed. F.E. Browder), Proc.Symp. Pure Math. 28, Amer.Math.Soc., Providence, RI, 1976, 1-34.

[17] Yu. Il'yashenko, Centennial History of Hilbert's 16th Problem, Bull.Amer.Math.Soc. 39(2002), 301-354.

[18] J. Li, Hilbert's 16th problem and bifurcations of planar polnomial vector fields, Internat. J. Bifur.Chaos 13 (2003), 47-106.

[19] J.Llibre, Averaging theory and limit cycles for quadratic systems, Rad.Mat. 11 (2002/3) 215-228.

[20] J. Llibre, M.A. Teixeira and J. Torregrosa, Limit cycles bifurcating from a kdimensional isochronous center contained in $\mathbb{R}^{n}$ with $k \leq n$, Math. Phys. Anal. Geom. 10 (2007), 237-249.

[21] L. Mazzi and M.A. Sabatini, Characterization of centres via first integrals. Advanced topics in the theory of dynamical systems, (Trento, 1987), 165177, Notes Rep. Math. Sci. Engrg. 6 Academic Press, Boston, MA, (1989). 
[22] H. Poincaré, Mémoire sur les courbes définies par les équation différentielles, in: Oeuvreus de Henri Poincaré, Vol. I, Gauthier-Villars, Paris, (1951), 95-114. 161-191.

[23] J.A. Sanders and F. Verhulst, Averaging methods in nonlinear dynamical systems, Appl. Math. Sci. 59 Springer-Verlag, New York - Berlin - Heidelberg - Tokyo, (1985).

[24] F. Verhulst, Nonlinear Differential Equations and Dynamical systems, Universitext, Springer, Berlin Heidelberg, New York, 1991.

[25] M. Viano, J. Llibre and H. Giacomini, Arbitrary order bifurcation for perturbed Hamiltonian planar systems via the reciprocal of an integrating factor, Nonlinear Anal. 48 (2002), 117-136. 